
Not for reproduction, distribution or commercial use.

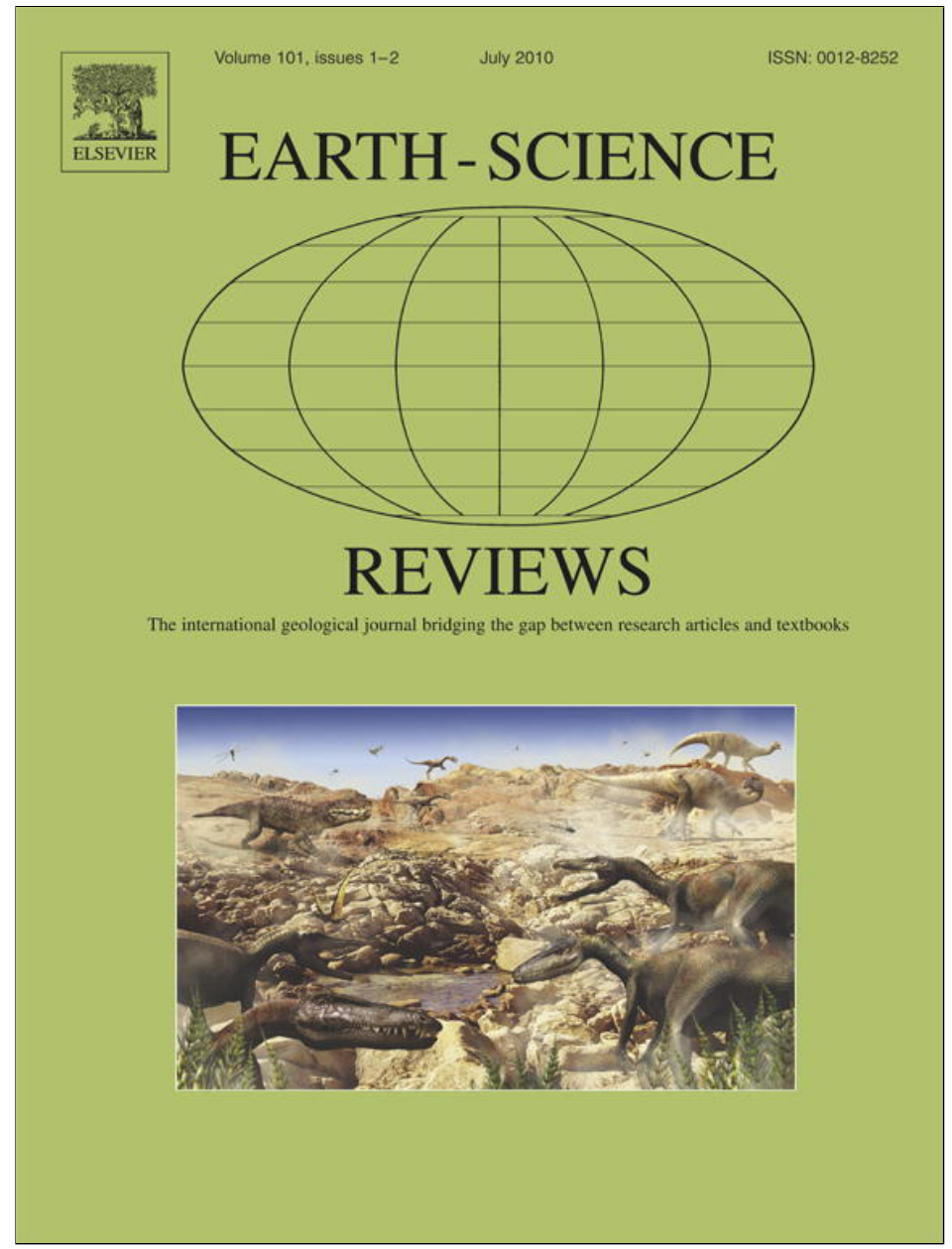

This article appeared in a journal published by Elsevier. The attached copy is furnished to the author for internal non-commercial research and education use, including for instruction at the authors institution and sharing with colleagues.

Other uses, including reproduction and distribution, or selling or licensing copies, or posting to personal, institutional or third party websites are prohibited.

In most cases authors are permitted to post their version of the article (e.g. in Word or Tex form) to their personal website or institutional repository. Authors requiring further information regarding Elsevier's archiving and manuscript policies are encouraged to visit:

http://www.elsevier.com/copyright 


\title{
The origin and early radiation of dinosaurs
}

\author{
Stephen L. Brusatte ${ }^{\mathrm{a}, \mathrm{b}, *}$, Sterling J. Nesbitt ${ }^{\mathrm{a}, \mathrm{b}, \mathrm{c}}$, Randall B. Irmis ${ }^{\mathrm{d}}$, Richard J. Butler ${ }^{\mathrm{e}}$, \\ Michael J. Benton ${ }^{\mathrm{f}}$, Mark A. Norell ${ }^{\mathrm{a}, \mathrm{b}}$ \\ a Division of Paleontology, American Museum of Natural History, Central Park West at 79th Street, New York, NY 10024, USA \\ ${ }^{\mathrm{b}}$ Department of Earth and Environmental Sciences, Columbia University, New York, NY, USA \\ c Jackson School of Geosciences, The University of Texas at Austin, Austin, TX 78712, USA \\ d Utah Museum of Natural History and Department of Geology \&' Geophysics, University of Utah, Salt Lake City, UT 84112, USA \\ e Bayerische Staatssammlung für Paläontologie und Geologie, Richard-Wagner-Str. 10, 80333 Munich, Germany \\ ${ }^{\mathrm{f}}$ Department of Earth Sciences, University of Bristol, Wills Memorial Building, Queens Road, Bristol BS8 1RJ, UK
}

\section{A R T I C L E I N F O}

\section{Article history:}

Received 1 May 2009

Accepted 20 April 2010

Available online 4 May 2010

\section{Keywords:}

dinosaurs

diversification

evolution

Jurassic

paleontology

Triassic

\begin{abstract}
A B S T R A C T
Dinosaurs were remarkably successful during the Mesozoic and one subgroup, birds, remain an important component of modern ecosystems. Although the extinction of non-avian dinosaurs at the end of the Cretaceous has been the subject of intense debate, comparatively little attention has been given to the origin and early evolution of dinosaurs during the Late Triassic and Early Jurassic, one of the most important evolutionary radiations in earth history. Our understanding of this keystone event has dramatically changed over the past 25 years, thanks to an influx of new fossil discoveries, reinterpretations of long-ignored specimens, and quantitative macroevolutionary analyses that synthesize anatomical and geological data. Here we provide an overview of the first 50 million years of dinosaur history, with a focus on the large-scale patterns that characterize the ascent of dinosaurs from a small, almost marginal group of reptiles in the Late Triassic to the preeminent terrestrial vertebrates of the Jurassic and Cretaceous. We provide both a biological and geological background for early dinosaur history. Dinosaurs are deeply nested among the archosaurian reptiles, diagnosed by only a small number of characters, and are subdivided into a number of major lineages. The first unequivocal dinosaurs are known from the late Carnian of South America, but the presence of their sister group in the Middle Triassic implies that dinosaurs possibly originated much earlier. The three major dinosaur lineages, theropods, sauropodomorphs, and ornithischians, are all known from the Triassic, when continents were joined into the supercontinent Pangaea and global climates were hot and arid. Although many researchers have long suggested that dinosaurs outcompeted other reptile groups during the Triassic, we argue that the ascent of dinosaurs was more of a matter of contingency and opportunism. Dinosaurs were overshadowed in most Late Triassic ecosystems by crocodile-line archosaurs and showed no signs of outcompeting their rivals. Instead, the rise of dinosaurs was a two-stage process, as dinosaurs expanded in taxonomic diversity, morphological disparity, and absolute faunal abundance only after the extinction of most crocodile-line reptiles and other groups.
\end{abstract}

(c) 2010 Elsevier B.V. All rights reserved.

\section{Contents}

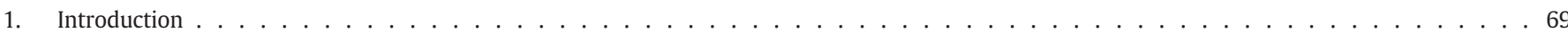

2. The biological setting for the origin of dinosaurs . . . . . . . . . . . . . . . . . . . . . . . . . . . . . . . . . . 70

2.1. Archosauria: the ruling reptiles . . . . . . . . . . . . . . . . . . . . . . . . . . . . . . . . . . . . 70

2.2. Avemetatarsalia: the "bird-line" of archosaur phylogeny . . . . . . . . . . . . . . . . . . . . . . . . . . . . . . 71

2.3. Dinosauria: definition . . . . . . . . . . . . . . . . . . . . . . . . . . . . . . . . . . 71

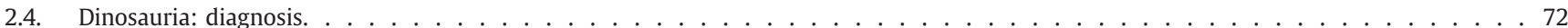

2.4.1. Character states that consistently diagnose Dinosauria. . . . . . . . . . . . . . . . . . . . . . . . . . . . . . . . . . . . 73

2.4.2. Character states that might diagnose Dinosauria. . . . . . . . . . . . . . . . . . . . . . . . . . 73

2.4.3. Character states that clearly do not diagnose Dinosauria . . . . . . . . . . . . . . . . . . . . . . . . . . . . . 74

2.4.4. Feathers: a dinosaur innovation? . . . . . . . . . . . . . . . . . . . . . . . . . . . . . . . . 74

\footnotetext{
* Corresponding author. Division of Paleontology, American Museum of Natural History, Central Park West at 79th Street, New York, NY 10024, USA. Tel.: +1 815 579 2682. E-mail address: sbrusatte@amnh.org (S.L. Brusatte).
} 
3. Geological setting for the origin of dinosaurs . . . . . . . . . . . . . . . . . . . . . . . . . . . . . . . . 75

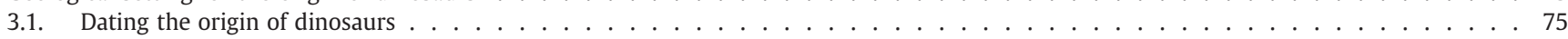

3.2. The paleoenvironment of early dinosaurs . . . . . . . . . . . . . . . . . . . . . . . . . . . . . . . . . . 76

3.3. Early dinosaur-bearing formations . . . . . . . . . . . . . . . . . . . . . . . . . . . . . . . . . . . . 77

4. Dinosaurs of the Late Triassic and Early Jurassic . . . . . . . . . . . . . . . . . . . . . . . . . . . . . . . . . . . . . 77

4.1. Ambiguous taxa: Eoraptor and Herrerasauridae . . . . . . . . . . . . . . . . . . . . . . . . . . . . . . . . . . . . . . 77

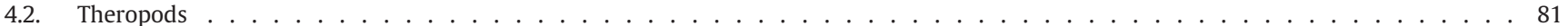

4.2.1. Late Triassic theropods - fossil record and distribution . . . . . . . . . . . . . . . . . . . . . . . . . . . . . . . 81

4.2.2. Late Triassic theropods - paleobiology. . . . . . . . . . . . . . . . . . . . . . . . . . . . . . . . . . 81

4.2.3. Early Jurassic theropods - fossil record and distribution . . . . . . . . . . . . . . . . . . . . . . . . . . . . . . . 82

4.2.4. Early Jurassic theropods - paleobiology . . . . . . . . . . . . . . . . . . . . . . . . . . . . . 82

4.2.5. Theropods across the Triassic/Jurassic boundary . . . . . . . . . . . . . . . . . . . . . . . . . . . . . . 82

4.3. Sauropodomorphs. . . . . . . . . . . . . . . . . . . . . . . . . . . . . . . . . 83

4.3.1. Late Triassic sauropodomorphs - fossil record and distribution . . . . . . . . . . . . . . . . . . . . . . . . . . . . . 83

4.3.2. Late Triassic sauropodomorphs - paleobiology . . . . . . . . . . . . . . . . . . . . . . . . . . . . . . . . . . 83

4.3.3. Early Jurassic sauropodomorphs - fossil record and distribution . . . . . . . . . . . . . . . . . . . . . . . . 84

4.3.4. Early Jurassic sauropodomorphs - paleobiology . . . . . . . . . . . . . . . . . . . . . . . . . . . . . 84

4.3.5. Sauropodomorphs across the Triassic/Jurassic boundary. . . . . . . . . . . . . . . . . . . . . . . . . . 84

4.4. Ornithischians. . . . . . . . . . . . . . . . . . . . . . . . . . . . . . . . . 84

4.4.1. Late Triassic ornithischians - fossil record and distribution . . . . . . . . . . . . . . . . . . . . . . . . . . . . 84

4.4.2. Late Triassic ornithischians - ghost lineages and diversity. . . . . . . . . . . . . . . . . . . . . . . . . . . . . . . 84

4.4.3. Late Triassic ornithischians - paleobiology . . . . . . . . . . . . . . . . . . . . . . . . . . . . . . 85

4.4.4. Early Jurassic ornithischians - fossil record and distribution . . . . . . . . . . . . . . . . . . . . . . . . . . . . . 85

4.4.5. Early Jurassic ornithischians - paleobiology . . . . . . . . . . . . . . . . . . . . . . . . . . . . . . 86

4.4.6. Ornithischians across the Triassic/Jurassic boundary . . . . . . . . . . . . . . . . . . . . . . . . 86

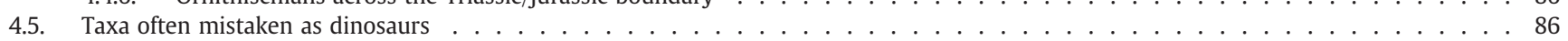

5. The dinosaur radiation: a historical review . . . . . . . . . . . . . . . . . . . . . . . . . . . . . . . . . 87

6. The macroevolutionary pattern of the dinosaur radiation . . . . . . . . . . . . . . . . . . . . . . . . . . . . . . . . . 88

6.1. Introduction . . . . . . . . . . . . . . . . . . . . . . . . . . . . . 88

6.2. Lineage origination, cladogenesis, and phylogeny . . . . . . . . . . . . . . . . . . . . . . . . . . . . . . . . 88

6.3. Taxonomic diversity and significant diversification shifts . . . . . . . . . . . . . . . . . . . . . . . . . . . . . . 89

6.4. Morphological disparity and morphospace occupation . . . . . . . . . . . . . . . . . . . . . . . . . . . . . . 90

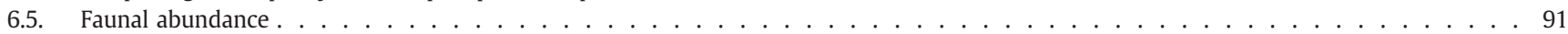

6.6. Rates of morphological change . . . . . . . . . . . . . . . . . . . . . . . . . . . . . . . . . . . . . . . . . 91

7. The evolutionary radiation of dinosaurs: current status. . . . . . . . . . . . . . . . . . . . . . . . . . . . . . . . . 91

8. The evolutionary radiation of dinosaurs: future directions . . . . . . . . . . . . . . . . . . . . . . . . . . . . . . . . . . . . . . 92

Acknowledgements. . . . . . . . . . . . . . . . . . . . . . . . . . . . . . . . . . . . . . . . . . 93

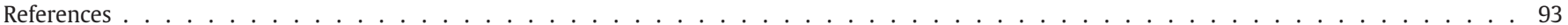

\section{Introduction}

Dinosaurs are icons of prehistory, and remain an important part of the modern world in the form of some 10,000 living species of birds. Although the extinction of non-avian dinosaurs at the end of the Cretaceous Period ( $65 \mathrm{Ma}$ ) has long been a focus of fascination and debate, the origin and early diversification of dinosaurs is not nearly as well understood. During the past 25 years, numerous new fossils, reinterpretations of long-forgotten specimens, and numerical analyses have significantly revised our understanding of this major macroevolutionary event, which is one of the most profound and important evolutionary radiations in the history of life.

In particular, new fossil material from Argentina (Sereno and Novas, 1992; Sereno et al., 1993; Martinez and Alcober, 2009), Brazil (Langer et al., 1999; Leal et al., 2004; Ferigolo and Langer, 2007), Africa (Yates and Kitching, 2003; Butler et al., 2007; Nesbitt et al., 2010; Yates et al., 2010), Europe (Dzik, 2003), and southwestern North America (Irmis et al., 2007a; Nesbitt et al., 2009b) has clarified the relationships of the first dinosaurs and their close relatives. Reanalysis of existing specimens has improved our understanding of character evolution on the lineage leading to Dinosauria (e.g., Sereno and Arcucci, 1994a,b; Langer and Benton, 2006; Brusatte et al., 2010b) and has changed our understanding of the distribution of early dinosaurs in time and space (Parker et al., 2005; Irmis et al., 2007b; Nesbitt et al., 2007). Most recently, quantitative analyses, which take into account this avalanche of new morphological and geological data, have examined in unprecedented detail the macroevolutionary, biogeographical, and paleoecological changes associated with the rise of dinosaurs (e.g., Brusatte et al., 2008a,b; Nesbitt et al., 2009b, 2010).

In this paper, we summarize current knowledge on the origin and early diversification of dinosaurs during the first 50 million years of their evolutionary history, from the Triassic through the Early Jurassic. Our aim is to provide a comprehensive synopsis of early dinosaur evolution, which may be of interest not only to specialists on dinosaurs or early Mesozoic earth history, but paleontologists, geologists, evolutionary biologists, and educators in general. As such, we frame our review in broad strokes, and provide information on the biological, geological, and evolutionary backdrop to early dinosaur history. We review the relationships of dinosaurs to other reptiles, define dinosaurs and discuss the anatomical features that distinguish them from other groups, summarize the early history of the major dinosaur clades, and discuss the physical and climatic background of early dinosaur faunas. We close by integrating this information into a comprehensive picture of the large-scale macroevolutionary patterns that characterize the origin and ascent of dinosaurs.

While our paper was in review, an independent summary of dinosaur origins was published by Langer et al. (2010). As these two manuscripts were written independently and at the same time, we do not discuss the conclusions of Langer et al. (2010) here, but note that the two papers largely complement each other in the discussion of early dinosaur evolution. 


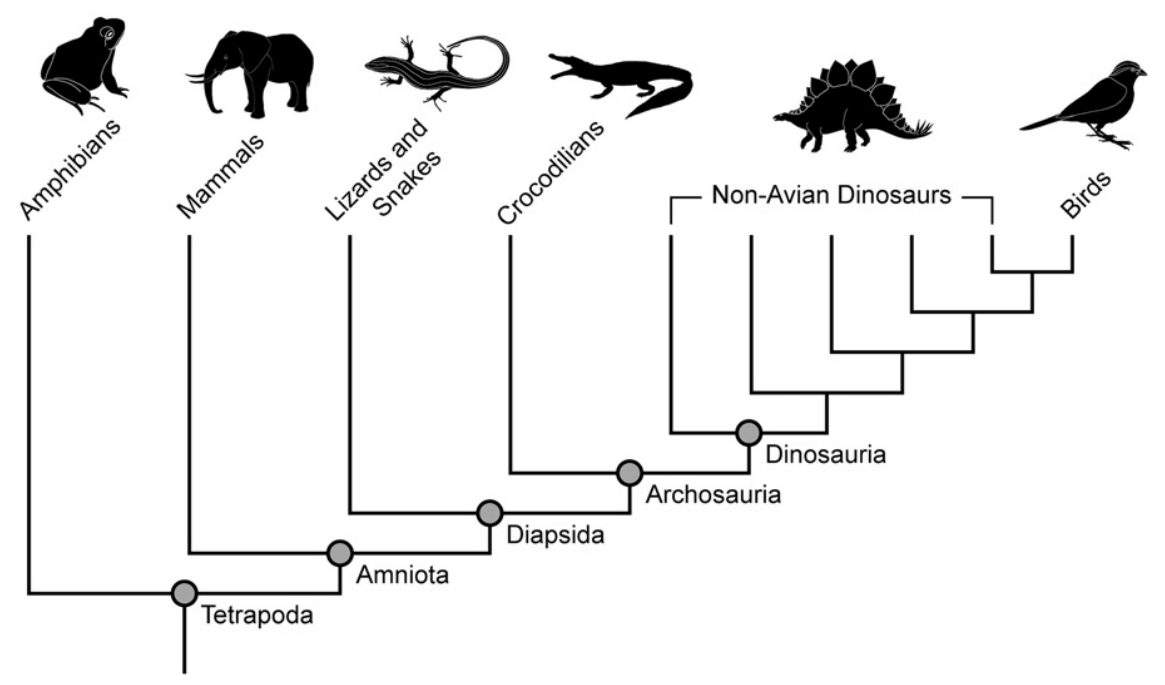

Fig. 1. A cladogram (phylogenetic or genealogical tree) of the major groups of tetrapods, the land-living vertebrates. Dinosaurs, including their avian descendants, are deeply nested within the Archosauria, a group that also includes crocodiles and their kin. Silhouettes not to scale. Cladogram delineated by Simon Powell, University of Bristol.

\section{The biological setting for the origin of dinosaurs}

\subsection{Archosauria: the ruling reptiles}

Dinosaurs are members of a speciose clade of vertebrates called the Archosauria (the "ruling reptiles": Cope, 1869), which includes birds, crocodylians, and their extinct relatives (note that we follow the definition of Archosauria as a crown group, consisting of birds, crocodiles, and all descendants of their most recent common ancestor, sensu Gauthier, 1986). Archosaurs are deeply nested within the radiation of land-living vertebrates, and themselves are a subgroup of diapsid reptiles (a more inclusive clade that also includes lizards, snakes, and possibly turtles: Fig. 1; Benton, 2005). The archosaur lineage originated approximately 245 million years ago, just a few million years after the devastating Permo-Triassic mass extinction. This extinction was the most profound period of mass death in geological history and is estimated to have wiped out up to $75-95 \%$ of all species (Raup, 1979; Stanley and Yang, 1994; Benton, 2003; Erwin, 2006; Clapham et al., 2009). In its aftermath, ecosystems reshuffled and entirely new groups of organisms arose and diversified, including "modern" lineages such as turtles, mammals, lepidosaurs, and archosaurs (e.g., Benton et al., 2004; Sahney and Benton, 2008).
The archosaur lineage diversified rapidly after its origination at the beginning of the Triassic (Nesbitt, 2003 see also Kubo and Benton, 2009). One of the oldest unequivocal archosaurs, Arizonasaurus, is known from the Anisian (ca. $243 \mathrm{Ma}$ ) of the southwestern United States (Nesbitt, 2003, 2005). It is a derived member of the "crocodile line" of archosaur phylogeny (Crurotarsi, also known as Pseudosuchia), which along with the "bird line" (alternatively known as Avemetatarsalia, Ornithodira, or Ornithosuchia) is one of the two major subdivisions of the archosaur clade (Fig. 2). The derived position of Arizonasaurus within Crurotarsi indicates that several other archosaur lineages extend back into the Middle Triassic, but the archosaur fossil record of this time is poor. During the Late Triassic, archosaurs of both major subgroups were exceptionally abundant in ecosystems across the globe. This period of time, from approximately 235-201 million years ago, witnessed the evolution of several morphologically distinctive archosaur clades that filled a variety of ecological roles (Nesbitt, 2007; Brusatte et al., 2008a; Nesbitt et al., 2010). Most of these groups, such as the long-snouted and semi-aquatic phytosaurs, the heavily armored aetosaurs, the sleek and predatory ornithosuchids, and the predatory and omnivorous "rauisuchians," became extinct by the end of the Triassic. Only the pterosaurs and dinosaurs, from the bird line, and the crocodylomorphs, derived members of the crocodile line, survived into the Jurassic.

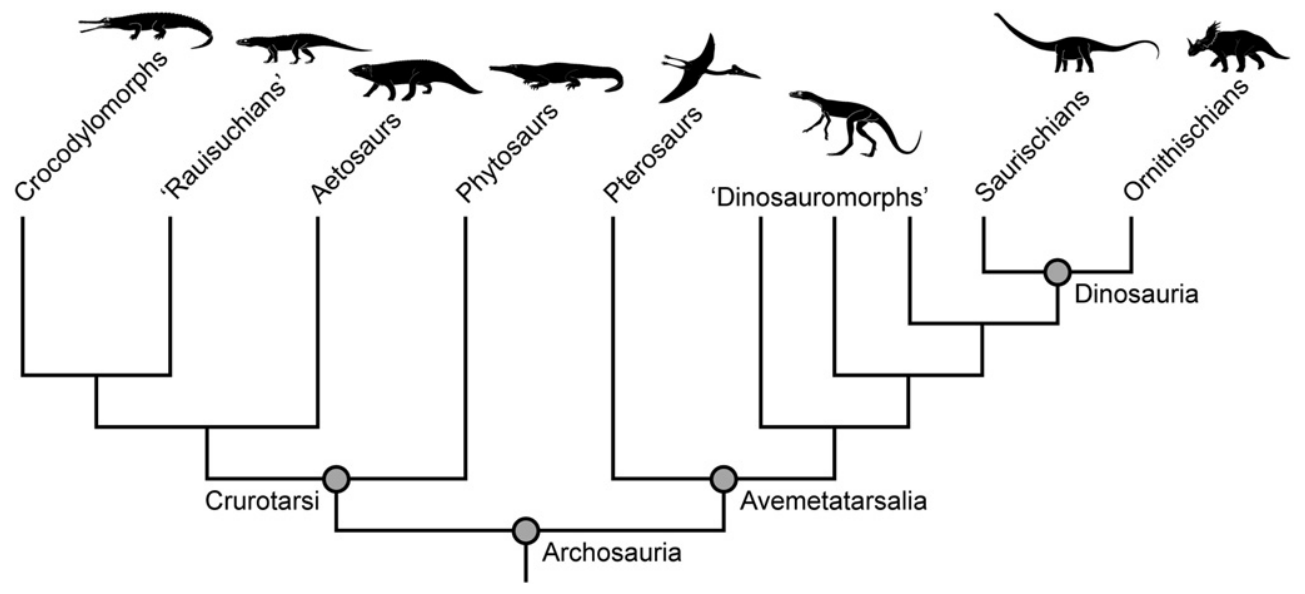

Fig. 2. A cladogram of the major groups of archosaurs. Archosauria is divided into two major groups, the crocodile line (Crurotarsi) and the bird line (Avemetatarsalia). The crocodile line is further subdivided into several subgroups (the long-snouted and semi-aquatic phytosaurs, the heavily armored aetosaurs, the mostly predatory rauisuchians, and true crocodylomorphs), whereas the bird line includes dinosaurs, birds, and a handful of close "dinosauromorph" cousins. Silhouettes not to scale. Cladogram delineated by Simon Powell, University of Bristol. 


\subsection{Avemetatarsalia: the "bird-line" of archosaur phylogeny}

Dinosaurs belong to Avemetatarsalia (an essentially equivalent group is also known as Ornithodira, or in the older literature, Ornithosuchia), the "bird line" of archosaur phylogeny. Extant birds, the only living members of this subgroup, are descended from theropod dinosaurs (e.g., Gauthier, 1986; Padian and Chiappe, 1998). However, several extinct Mesozoic taxa also belong to the bird-line lineage, including the non-avian dinosaurs, pterosaurs (flying reptiles), and a handful of early non-dinosaurian dinosauromorphs that are the closest relatives of dinosaurs (herein referred to as "basal dinosauromorphs"). Only a small sample of basal dinosauromorphs has been discovered. These range in age from the Middle-Late Triassic and are known primarily from small, fragmentary, and incomplete specimens often missing entire regions of the skeleton. Most of these taxa resemble small predatory dinosaurs in their overall anatomy (e.g., Lagerpeton and Marasuchus: Sereno and Arcucci, 1994a,b), whereas recently discovered taxa such as Silesaurus (Dzik, 2003), Sacisaurus (Ferigolo and Langer, 2007), and Asilisaurus (Nesbitt et al., 2010) were quadrupedal herbivores or omnivores whose teeth superficially resemble those of ornithischian dinosaurs. Phylogenetic analyses indicate that these herbivorous taxa form their own distinct clade, Silesauridae, which is the immediate sister taxon (closest relative) of Dinosauria (Irmis et al., 2007a; Brusatte et al., 2008a, 2010b; Nesbitt et al., 2009b, 2010; Langer et al., 2010). The recent discovery of Late Triassic representatives of these groups (e.g., Dzik, 2003 and Irmis et al., 2007a) demonstrates that they co-existed with dinosaurs for at least 15 million years.

\subsection{Dinosauria: definition}

As with any group of organisms, the designation of what does and does not constitute a dinosaur (Fig. 3) is a matter of definition. Traditional taxonomists, beginning with Owen (1842), defined Dinosauria based on a set of shared anatomical features. Fossil reptiles were considered dinosaurs if they possessed these characteristics, which historically have related to size, posture, and locomotion (see below). However, most modern systematists define groups of organisms based on ancestry instead of the possession of "essential" characters (e.g., de Queiroz and Gauthier, 1990, 1992; Sereno, 2005). Under such a system, known as phylogenetic taxonomy, an animal is a dinosaur only if it falls out in a certain place on the tree of life. Anatomical characteristics are used to reconstruct the genealogical tree, and serve to diagnose groups, but their possession is not an essential requirement for group membership.

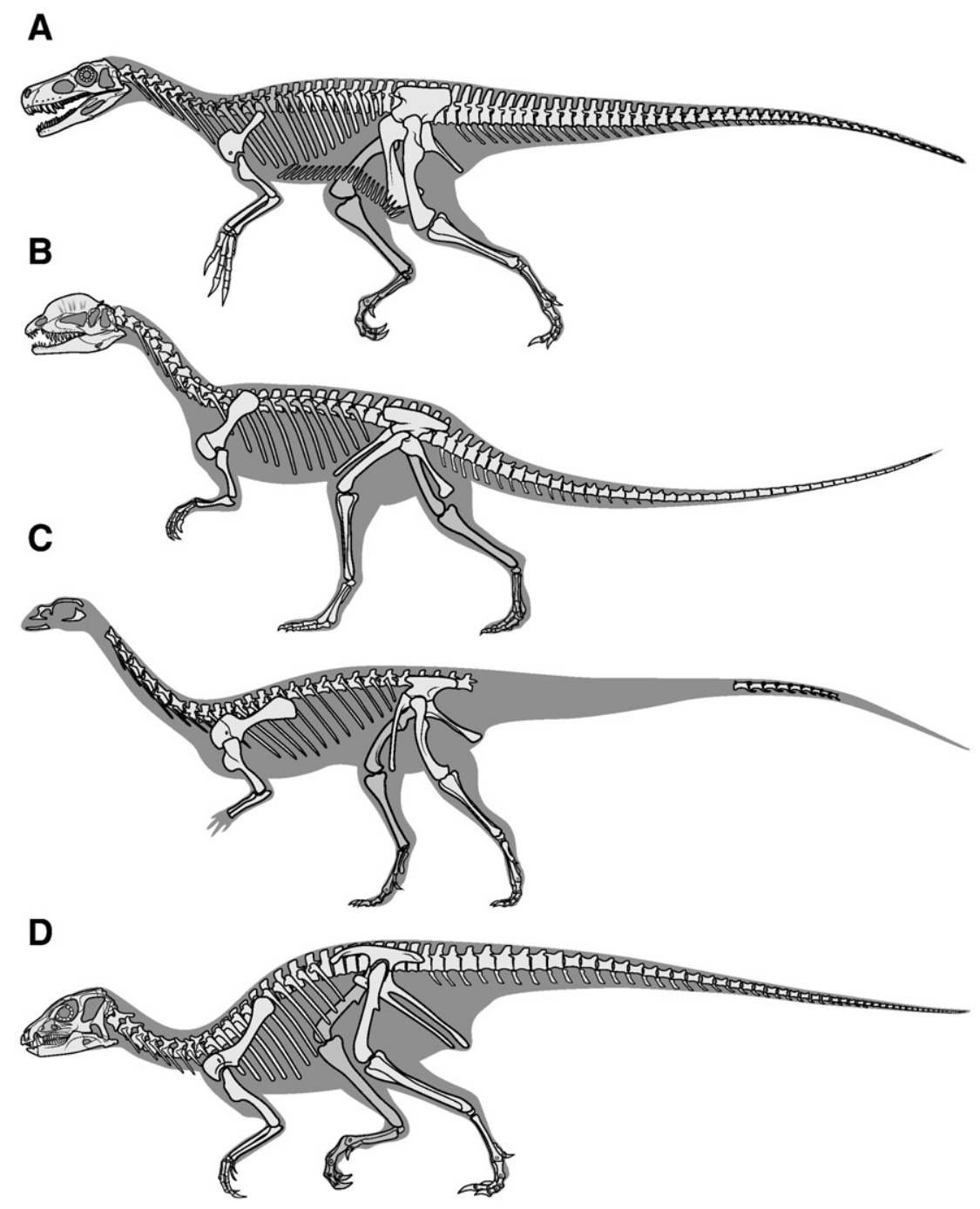

Fig. 3. Skeletal reconstructions of four Late Triassic-Early Jurassic dinosaurs, representing the major subgroups of early dinosaurs. These reconstructions are designed to provide a general guide to early dinosaur skeletal anatomy, and should not be used for fine-scale anatomical comparison or character state scoring in phylogenetic analysis. A, Herrerasaurus ischigualastensis (Dinosauria incertae sedis, possibly a theropod or stem saurischian outside the theropod + sauropodomorph clade); B, Dilophosaurus wetherilli (Theropoda); C, Saturnalia tupiniquim (Sauropodomorpha); D, Heterodontosaurus tucki (Ornithischia). Reconstructions delineated by Frank Ippolito, American Museum of Natural History. 
Under the phylogenetic taxonomy system, Dinosauria is defined as the least inclusive clade containing Triceratops horridus and Passer domesticus (Padian and May, 1993; Sereno, 1998; Sereno et al., 2005). This definition is sometimes phrased as: "Triceratops horridus, Passer domesticus, and all descendants of their most recent common ancestor." Under this definition, an organism is only a dinosaur if it is a member of the group on a phylogeny (cladogram) that can be traced down to the node representing the common ancestor of Triceratops and modern birds (of which Passer is an exemplar). Such a definition is not specific to a certain phylogenetic tree-which is always a hypothesis that can be overturned by new discoveries and interpretations-but rather can be applied to any phylogeny. However, under different phylogenies, different organisms may or may not be dinosaurs depending on their relationships. It is important to remember that this has nothing to do with anatomical features (other than the fact that anatomy is usually used to reconstruct the phylogeny). Most current phylogenetic analyses find silesaurids to fall just outside of the defined dinosaur group, even though both possess many features that were once thought to be unique to dinosaurs. However, in the future, newer phylogenetic work may show these genera to fall inside the dinosaur group, thus necessitating their classification as dinosaurs. In this way, the phylogenetic taxonomy system is flexible in dealing with revised hypotheses of relationships.

Some researchers use a slightly different definition of Dinosauria: "the least inclusive clade containing Megalosaurus and Iguanodon." In essence this definition replaces Triceratops and Passer with two alternative specifier taxa. Megalosaurus and Iguanodon are preferred by some because they were the first two dinosaurs named, and were instrumental in shaping Richard Owen's (1842) concept of Dinosauria. However, we prefer using Triceratops and Passer for two reasons: these specifiers were used in the first phylogenetic definition of Dinosauria (Padian and May, 1993) and Megalosaurus is a poorly understood and fragmentary taxon that has only recently been redescribed in detail (Benson et al., 2008; Benson, 2010b).

\subsection{Dinosauria: diagnosis}

Although Dinosauria is defined on ancestry and not anatomical characters, there is still a diagnostic set of features that is characteristic of dinosaurs and unknown in other organisms. These characters are said to diagnose dinosaurs rather than define them, just as medical symptoms can be diagnostic of a disease but no disease is rigidly defined by a set of symptoms. In a cladistic sense, these diagnostic features are shared derived characters (synapomorphies) that support Dinosauria as a unique natural group (a monophyletic clade) on the tree of life.

Owen (1842) first recognized Dinosauria as a distinctive group, containing Megalosaurus, Iguanodon, and Hylaeosaurus, based on several shared features of the hips (three sacral vertebrae), limbs, and body posture (upright stance) (Cadbury, 2002). Over time, nearly a thousand new non-avian dinosaurs have since been added to this original triumvirate. In doing so, new characters were identified as additional distinctive dinosaur features whereas some of Owen's original characters were dismissed as inaccurate or also observed in other non-dinosaur fossil reptiles. By the end of the 19th century paleontologists recognized two major groups of dinosaurs: the "lizard-hipped" saurischians, which include carnivorous dinosaurs such as Megalosaurus and the long-necked herbivorous sauropods, and the "bird-hipped" ornithischians, which include an array of armored, ornamented, and large-bodied herbivores such as Iguanodon and Triceratops (Seeley, 1888). These groups are still recognized as the two major subdivisions of dinosaurs. However, for much of the 19th and 20th centuries paleontologists considered saurischians and ornithischians to represent separate lineages, which independently diverged long ago from separate "thecodont" (a term applied to an illdefined assemblage of primitive archosaurs) ancestors and thus were not particularly closely related (e.g., Colbert, 1964; Charig et al., 1965; Romer, 1966). Thus, in a cladistic sense, dinosaurs were seen as a polyphyletic (non-natural) group.

In a seminal paper published in 1974, Bakker and Galton persuasively argued that saurischians and ornithischians were not distant relatives, but rather could be united within a monophyletic Dinosauria. In essence, they resurrected Owen's (1842) original concept of a single, unique natural group of Mesozoic vertebrates that could be distinguished from all other organisms based on their possession of shared derived characters. Several anatomical features shared by saurischians and ornithischians were recognized by Bakker and Galton (1974:168-169), including upright and fully erect posture, an enlarged deltopectoral crest on the humerus, a "specialized" hand, a perforated acetabulum (hip socket), a well-developed fourth trochanter on the femur, a lesser trochanter on the femur, and an ankle joint in which the proximal tarsals (astragalus and calcaneum) were "fixed immovably on the ends of the tibia and fibula, [resulting in a] simple unidirectional hinge between the astragalus-calcaneum and distal tarsals." To Bakker and Galton (1974), these shared skeletal features were unlikely to have arisen by convergent evolution, but rather are shared characters that ornithischians and saurischians inherited from a common ancestor. Under Bakker and Galton's (1974) conception, Dinosauria also included a living group of descendants: the birds. This was not a new idea: it had been proposed as early as the 1860s (e.g., Huxley, 1868, 1870a,b), but had fallen out of favor until the pioneering studies of John Ostrom in the 1960s (e.g., Ostrom, 1969, 1973).

Although dinosaur monophyly was controversial to some (e.g., Charig, 1976a,b; Thulborn, 1975; Chatterjee, 1982; Charig, 1993), most vertebrate paleontologists enthusiastically accepted Bakker and Galton's (1974) evidence as overwhelming (e.g., Bonaparte, 1975; Benton, 1984, 1985; Cruickshank and Benton, 1985; Padian, 1986; Sereno, 1986). The advent of numerical cladistic analyses in the mid 1980s crystallized support for both dinosaur monophyly and the hypothesis that birds evolved from theropod dinosaurs (e.g., Gauthier, 1986; Benton and Clark, 1988; Sereno, 1991a). Today, higher-level phylogenetic analyses continue to find robust support for dinosaur monophyly (e.g., Juul, 1994; Benton, 1999, 2004; Sereno, 1999; Ezcurra, 2006; Langer and Benton, 2006; Irmis et al., 2007a; Brusatte et al., 2008a; Nesbitt et al., 2009b; Brusatte et al., 2010b; Nesbitt et al., 2010), although the exact characters diagnosing the dinosaur group continue to change as new fossils are found and old ideas are reinterpreted.

Over 50 characters have been cited as dinosaur synapomorphies in both pre-cladistic and cladistic studies (Bakker and Galton, 1974; Benton, 1984; Gauthier, 1986; Benton and Clark, 1988; Novas, 1989; Sereno, 1991a; Novas, 1992; Sereno and Novas, 1994; Novas, 1996; Benton, 1999; Sereno, 1999; Fraser et al., 2002; Langer and Benton, 2006; Irmis et al., 2007a; Nesbitt et al., 2009b, 2010; Brusatte et al., 2010b). Potential dinosaur characteristics are distributed throughout the body. However, very few characters of the skull diagnose Dinosauria as a whole, and most unique dinosaur features relate to the limbs and girdles. This pattern reflects two factors. First, the limbs and girdles are heavily modified compared to close relatives, presumably a result of a transition from a facultative quadrupedal to an obligate bipedal posture on the lineage leading to dinosaurs. Second, many close dinosaur outgroup fossils are missing skulls and hands but preserve nearly complete limbs and girdles, thus enabling detailed study of these structures.

Of the pool of potential dinosaur synapomorphies, characters can be partitioned into three categories: (1) character states that consistently diagnose Dinosauria; (2) character states that might diagnose Dinosauria, but whose distribution in close outgroup taxa remains unknown or ambiguous; and (3) character states that clearly do not diagnose Dinosauria, usually because they have subsequently been identified in other organisms. Recently, a striking pattern has 
emerged. Few characters are unique to Dinosauria, and many longstanding dinosaur synapomorphies are actually found in other archosaur taxa. Most of these seem to represent independent acquisitions (convergences), underscoring the remarkable similarity of distantly related archosaurs that lived during the Triassic Period (e.g., Nesbitt and Norell, 2006).

In the following sections we briefly discuss and review the most frequently cited character states that may or may not diagnose Dinosauria (Fig. 3).

\subsubsection{Character states that consistently diagnose Dinosauria}

2.4.1.1. Elongated deltopectoral crest. The deltopectoral crest of the humerus, a muscle attachment site for the deltoid and pectoralis muscles of the arms and chest, extends along $30-40 \%$ of the humerus in both saurischians and ornithischians. In nearly all other archosaurs, including the close dinosaur outgroups Marasuchus and silesaurids, the deltopectoral crest is shorter and restricted to the proximal region of the humerus. An elongated deltopectoral crest is also convergently present in the basal archosauriform Erythrosuchus (Gower, 2003) and the crurotarsan archosaur Yarasuchus (Sen, 2005).

2.4.1.2. Open acetabulum. The acetabulum, the joint surface on the pelvis that articulates with the femur, is backed by a medial wall of bone in most reptiles. However, in most dinosaurs the acetabulum is "open" like a window, with no bounding wall. An open acetabulum has long been cited as a dinosaur synapomorphy and is clearly present in ornithischians, theropods, and nearly all sauropodomorphs (except for the basal sauropodomorphs Panphagia and Saturnalia, which have a "partially" open acetabulum). This character is often specified in phylogenetic analysis by reference to the ventral margin of the ilium (e.g., Irmis et al., 2007a: character 65). In taxa with an open acetabulum the ventral margin of the ilium is distinctly concave. In the closest relatives of dinosaurs, Silesaurus and Marasuchus, the ventral margin of the ilium is essentially straight, with at most a small concave divot, and this condition has been referred to as an incipiently open acetabulum (e.g., Sereno and Arcucci, 1994b; see discussion in Novas, 1996). Although rare among archosauriforms, a concave ventral margin of the ilium is present in some crurotarsan archosaurs (e.g., Poposaurus: Weinbaum and Hungerbühler, 2007), including nearly all basal crocodylomorphs (e.g., Crush, 1984).

2.4.1.3. Temporal musculature extends anteriorly onto skull roof. The frontals of all early dinosaurs have a distinct fossa anterior to the supratemporal fenestra, which likely was an attachment site for the upper temporal musculature used to adduct (close) the lower jaw (Gauthier, 1986). Although most close dinosaur relatives lack cranial material, the well-preserved frontals of the early dinosauromorph Silesaurus do not have a fossa (Dzik, 2003), thus indicating that the extensive fossa is a dinosaur character. However, basal crocodylomorphs also bear a distinct fossa on the frontal anterior to the supratemporal fenestra.

2.4.1.4. Epipophyses on the cervical vertebrae. Epipophyses are projections of bone, likely for muscle and ligament attachment, which protrude from the dorsal surfaces of the postzygapophyses of the cervical vertebrae. All basal dinosaurs possess epipophyses (Langer and Benton, 2006), although the size, shape, length, and projection angle of these processes vary considerably (e.g., compare Coelophysis (Colbert, 1989) with the more derived theropod Majungasaurus (O'Connor, 2007)). Basal ornithischians (e.g., Heterodontosaurus) only have epipophyses on the anterior cervical vertebrae, whereas saurischians have epipophyses in nearly all cervical vertebrae (Langer and Benton, 2006). Epipophyses are not present in the closest relatives of dinosaurs (e.g., Marasuchus, Silesaurus), but are present in some crurotarsans (e.g., Lotosaurus and Revueltosaurus).
2.4.1.5. Articulation facet for fibula occupying less than $30 \%$ of the transverse width of the astragalus. Both bones of the lower hind limb, the tibia and the fibula, articulate with the astragalus bone of the ankle in archosaurs. In dinosaurs, the fibula only makes a restricted contact with the astragalus, such that the fibular articular facet of the astragalus is less than $30 \%$ of the transverse width of the astragalus itself. This feature is unique to dinosaurs and unknown in other archosaur groups.

2.4.1.6. Femoral fourth trochanter asymmetrical, with distal margin forming a steeper angle to the shaft. The caudofemoralis, one of the major muscles controlling the hindlimb, attaches to a rugose scar on the shaft of the femur called the fourth trochanter. Bakker and Galton (1974) first suggested that a modification of the fourth trochanter represents a shared derived character for dinosaurs. Although their original concept is not specific and no longer valid, basal dinosaurs do share an asymmetrical, crest-like fourth trochanter, in which the ventral portion of the scar is medially expanded relative to the dorsal portion. This morphology contrasts with the rounded, symmetrical fourth trochanter of Silesaurus, Marasuchus, and crurotarsan archosaurs, and thus is only present in dinosaurs. Theropod dinosaurs later re-evolve a symmetrical fourth trochanter, but this is independent of the condition seen in early dinosauromorphs.

2.1.4.7. Posterior process of the jugal bifurcated to articulate with the quadratojugal. The jugal bone, which forms the lateral "cheek" region of the skull underneath the eye, has a bifurcated posterior process in dinosaurs. This bifurcation receives the anterior prong of the quadratojugal, and presumably strengthens the contact between the two bones. In other archosaurs, including Silesaurus (Dzik and Sulej, 2007:fig. 18A), the single posterior process of the jugal either lies above or below the anterior process of the quadratojugal.

\subsubsection{Character states that might diagnose Dinosauria}

Fossil specimens of the closest relatives of Dinosauria, such as Lagerpeton, Marasuchus, Silesaurus, and early pterosaurs, are often incomplete and poorly preserved. Most of these lack skulls and hands, and when present these structures are often eroded, crushed, or fragmentary. Therefore, although dinosaurs possess many interesting and potentially diagnostic characters of the skulls and hands, these are difficult to evaluate because we cannot determine if the characters are present in the closest dinosaur relatives. They may represent true dinosaur synapmorphies, or they may characterize a more inclusive group but are currently unrecognized in other taxa due to missing data alone. The following characters fall into this category: postfrontal absent, ossified and paired sternal plates, reduced manual digits IV and $\mathrm{V}$, three or fewer phalanges in the fourth manual digit, and posttemporal foramen present.

In a similar vein, the following potential synapomorphies are absent in the proximal outgroups to dinosaurs, but their distribution within Dinosauria remains complicated. They may represent dinosaur synapomorphies, but further study is required.

2.4.2.1. Brevis fossa/shelf. In archosaurs, a portion of the caudofemoralis musculature, the caudofemoralis brevis, attaches to either the lateral or ventral portion of the posterior process of the ilium, just posterior to the acetabulum (Carrano and Hutchinson, 2002). Only a slight attachment scar for this muscle is present on the ilia of crurotarsans, whereas most dinosaurs have a distinct scar or fossa (= pocket) on either the lateral or ventral surface of the ilium. This fossa is usually referred to as the "brevis fossa," and its medial bounding rim the "brevis shelf" (Novas, 1996). However, the distribution of this character among dinosaurs and close outgroups is complex, and it is possible that not all conditions are homologous. For instance, the basal dinosaur Herrerasaurus lacks any kind of brevis fossa (contra Novas, 1993), whereas the non-dinosaur Silesaurus possesses a distinct fossa, 
which was likely acquired independently from that of dinosaurs since the basal silesaurid Asilisaurus lacks a fossa (Nesbitt et al., 2010). Furthermore, the basal condition among ornithischians is unclear: Heterodontosaurus lacks a lateral expression of the fossa, Eocursor has a shallow fossa on the ventral surface of the ilium, and Lesothosaurus has a distinct scar on the lateral surface of the ilium. Similarly, the condition in sauropodomorphs is unsettled: the basal taxon Panphagia has a deep fossa, the basal Saturnalia possesses a small fossa, and Plateosaurus lacks even a rudimentary fossa. Clearly this character deserves further study, and detailed comparisons of the brevis fossa are needed in order to assess its homology among taxa.

2.4.2.2. At least three sacral vertebrae. The number of sacral vertebrae, those vertebrae that articulate with the pelvis, has often been used as a character in phylogenetic analyses (e.g., Gauthier, 1986; Benton and Clark, 1988; Novas, 1996; Benton, 1999). The dinosauromorphs Lagerpeton and Marasuchus have two primordial sacral vertebrae, a character state that is usually optimized as the primitive condition among archosaurs. The recently discovered Silesaurus, a member of the sister taxon of Dinosauria, has three sacral vertebrae, whereas the basal dinosaurs Herrerasaurus and Staurikosaurus have only the two primordial sacrals, which has been considered as a reversal to the primitive condition (Novas, 1996). Basal ornithischians have as many as six (Heterodontosaurus), sauropodomorphs have at least three, and neotheropods have at least five sacral vertebrae. The identity of individual sacrals is often complex. Novas (1996) and Langer and Benton (2006) attempted to identify each sacral vertebra as a dorsosacral (a dorsal vertebra incorporated into the sacrum), a primordial sacral (a sacral homologous to the plesiomorphic two of Marasuchus and other tetrapods), or a caudosacral (a caudal vertebra incorporated into the sacrum). However, their methods for identifying sacral vertebrae have recently been questioned (Nesbitt, 2008). Given the varying numbers of sacrals in early dinosaurs and outstanding questions over the identification of individual sacrals, the number of sacral vertebrae at the root of Dinosauria has yet to be accurately determined.

\subsubsection{Character states that clearly do not diagnose Dinosauria}

Many characters once thought to diagnose dinosaurs have moved down the stem and now represent synapomorphies of more inclusive clades. This is a direct result of the discovery of several close dinosaur relatives, such as Asilisaurus (Nesbitt et al., 2010), Silesaurus (Dzik, 2003), Sacisaurus (Ferigolo and Langer, 2007), and Dromomeron (Irmis et al., 2007a; Nesbitt et al., 2009a), as well as the redescription of Marasuchus (Sereno and Arcucci, 1994b), Lagerpeton (Sereno and Arcucci, 1994a), and Eucoelophysis (Ezcurra, 2006; Nesbitt et al., 2007). These characters include: ectopterygoid dorsal to transverse flange of the pterygoid; posteroventrally oriented glenoid on the scapula and coracoid; reduced pubis/ischium contact; reduced ischiadic medioventral lamina; inturned femoral head; proximal femur with reduced medial tuberosity; anterior trochanter of the femur present; tibial descending process that fits posterior to the astragalar ascending process; flat to concave proximal calcaneum; presence of mesotarsal ankle; metatarsals II and IV subequal in length; and a distal end of metatarsal IV that is taller than wide.

As additional dinosauromorph taxa are discovered and redescribed and archosaur anatomy and phylogeny is studied in more detail, it is possible that some of the characters listed above as "consistent" dinosaur synapomorphies will also move down the stem. However, keeping in mind the large number of discoveries of the past 30 years, it is remarkable that many of Bakker and Galton's (1974) original diagnostic characters of Dinosauria still remain valid.

\subsubsection{Feathers: a dinosaur innovation?}

Without question, one of the largest surprises in paleontology in the last 15 years has been the discovery of feathers and feather-like structures in non-avian dinosaurs. These structures were first reported in small compsognathid theropods from the Early Cretaceous Yixian Formation of northern China (Ji and Ji, 1996; Chen et al., 1998). These structures are not true feathers, but rather small filamentous integumentary structures termed "protofeathers," a presumed evolutionary precursor to true feathers. Their nature had been disputed until the recent report that they contain color-bearing melanosomes exactly as in modern bird feathers (Zhang et al., 2010; see also Li et al., 2010). These finds were soon followed by the announcement of feathers of modern aspect, nearly indistinguishable from those in living birds, in a number of close bird relatives (Ji et al, 1998). The geologically oldest specimens to show feather-like structures include the theropod Pedopenna (Xu and Zhang, 2005) and two taxa belonging to the bizarre and poorly-known theropod clade Scansoriopterygidae (Zhang, et al., 2002; Zhang et al., 2008) from the Daohugou Formation, which may be as old as Middle Jurassic (Liu et al, 2006)."Feathered" non-avian dinosaurs, including tyrannosauroids (Xu et al., 2004), compsognathids (Goehlich et al., 2006; Ji et al., 2007), dromaeosaurs (Xu et al., 1999a,b; Ji et al., 2001), therizinosaurs (Xu et al., 1999a,b) and troodontids (Ji et al., 2005), continue to be described regularly.

Currently, the key question is: "how deep in the dinosaur family tree do feathers, or integumentary structures homologous with feathers, go?" Until recently, the occurrence of integumentary structures in dinosaurs outside Theropoda has been controversial. In 2002 an unusual specimen of the common Yixian ornithischian Psittacosaurus was described as possessing a comb-like structure of wavy bristle-like filaments on the tail (Mayr et al., 2002). Although the identity of these structures has been contested (one author even suggested they were a fossil plant associated with the specimen), observation of the specimen (by MAN) validates Mayr et al.'s (2002) interpretation. Unfortunately, the provenance of this specimen (removed from China illegally and in a foreign museum) makes it difficult and unethical to incorporate it into any informed scientific discussion (see Dalton, 2001; Long, 2003). Yet, recently another specimen, this time a heterodontosaurid ornithischian, was reported as possessing feather-like structures (Zheng et al., 2009). This taxon, Tianyulong, displays both the thick, wavy, bristle-like tail structures of Psittacosaurus, as well as more enigmatic integument (perhaps "protofeathers") in the neck area. Finally, it is worth pointing out that filamentous integumentary coverings have been reported in a variety of pterosaurs, flying reptiles which are close relatives of dinosaurs but outside of Dinosauria proper. These fossils, including specimens of Sordes pilosus (Sharov, 1970; Bakhurina and Unwin, 1995) and several specimens from the Yixian and Daohugou Formations (Lu, 2002; Wang et al., 2002; Ji and Yuan, 2002; Kellner et al., 2009; see Norell and Ellison, 2005) show incontrovertible evidence for such structures, but it is unclear whether these are homologous to bird feathers or even dinosaurian "protofeathers."

The earliest fossils that physically preserve integumentary structures, which are difficult to fossilize except in remarkable conditions, have been found in Middle Jurassic rocks. However, because these structures are found in a diversity of ornithischian and saurischian dinosaurs, there is little doubt that they were present in the ancestor of all dinosaurs, and probably the ancestor of dinosaurs and pterosaurs as well (and therefore present primitively in Ornithodira). Thus, filamentous, feather-like structures (true feathers or protofeathers) must have been present in Late Triassic dinosaurs. Could feather-like structures extend much deeper in the reptile phylogenetic tree? This is one of the most exciting questions of modern vertebrate paleontology, and its answer depends on both the discovery of additional remarkable fossils and the investigation of molecular and developmental evidence of structural feather proteins in extant non-dinosaurian archosaurs (e.g., crocodiles). Therefore, in summary, although more research needs to be completed, the hypothesis that keratinous feather-like coverings are homologous 
for Dinosauria and beyond seems reasonable at this time. The evolutionary (physiologic, sociobiologic, biomechanical and developmental) aspects of this are only beginning to be studied.

\section{Geological setting for the origin of dinosaurs}

\subsection{Dating the origin of dinosaurs}

Dinosaurs likely originated during the Middle Triassic (Nesbitt et al., 2010) and the first unequivocal dinosaur fossils are known from the late Carnian. However, much about the geological and temporal backdrop of early dinosaur history remains poorly understood (Fig. 4). A well-resolved chronostratigraphic framework is necessary to answer questions successfully about the tempo and mode of the origin of dinosaurs. This requirement has been one of the many challenges to developing a consensus on how and why dinosaurs became so successful during the early Mesozoic. In particular, there have been three major outstanding questions: (1) what are the ages and durations of the marine stages of the Late Triassic Period?; (2) how can these stages, which are defined using marine invertebrate biostratigraphy, be correlated to terrestrial dinosaur-bearing formations?; and (3) what are the numerical absolute ages of the principal terrestrial vertebrate assemblages that contain early dinosaurs?

The uncertainty surrounding the age and duration of the Carnian, Norian, and Rhaetian stages is a direct result of the lack of precise radioisotopic dates (Mundil, 2007). Although the most recent estimates indicate that the Late Triassic Epoch is over 30 million years long (e.g., Muttoni et al., 2004; Furin et al., 2006), there are only four published precise radioisotopic ages (Rogers et al., 1993; Riggs et al., 2003; Furin et al., 2006; Schaltegger et al., 2008) for this time period. The base of the Late Triassic is poorly dated: there are no precise radioisotopic ages from near the Ladinian-Carnian boundary and there is no published magnetostratigraphic record that crosses the boundary. An approximate age of $235 \mathrm{Ma}$ for the Ladinian-Carnian boundary has been interpolated using records from earlier in the Ladinian (e.g., Mundil et al., 1996; Muttoni et al., 1997; Mundil et al., 2003; Brack et al., 2005). The Carnian-Norian boundary is constrained by a new $\mathrm{U}-\mathrm{Pb}$ single crystal zircon age of $230.91 \pm 0.33 \mathrm{Ma}$ from the Upper Carnian marine section at Pignola, Italy (Furin et al., 2006). Biostratigraphic correlation of this section to magnestratigraphic records from elsewhere in the Tethys region place the Carnian-Norian boundary at between 227 and $228 \mathrm{Ma}$ (Furin et al., 2006: fig. 1), consistent with the Newark Astrochronological Polarity Timescale from eastern North America (Muttoni et al., 2004). The Norian is very poorly dated: there is only one published precise radioisotopic age (Riggs et al., 2003), and it is from terrestrial strata that cannot be directly correlated to the marine biostratigraphic events that define stage boundaries. Calibration of magnetostratigraphic records using palynomorph assemblages (e.g., Kent and Olsen, 1999; Muttoni et al., 2004) and magnetostratigraphy from a key marine section (Muttoni et al., 2010) indicate an age of 208-209 Ma for the Norian-Rhaetian boundary. Taken together, these data suggest that the Norian Stage has a duration of approximately $20 \mathrm{Ma}$, two-fifths the length of the entire Triassic Period. The end of the Rhaetian (Triassic-Jurassic boundary) is well constrained to between 202 and $201 \mathrm{Ma}$ by $\mathrm{U}-\mathrm{Pb}$ ages and magnetostratigraphic data (e.g., Kent and Olsen, 1999; Schoene et al., 2006; Schaltegger et al., 2008; Jourdan et al., 2009), with an estimated age of 201.3 Ma based on cyclostratigraphy (Whiteside et al., 2010).

The earliest known dinosauromorph-bearing assemblage is from the ?late Anisian Manda Formation of Tanzania (Nesbitt et al., 2010),

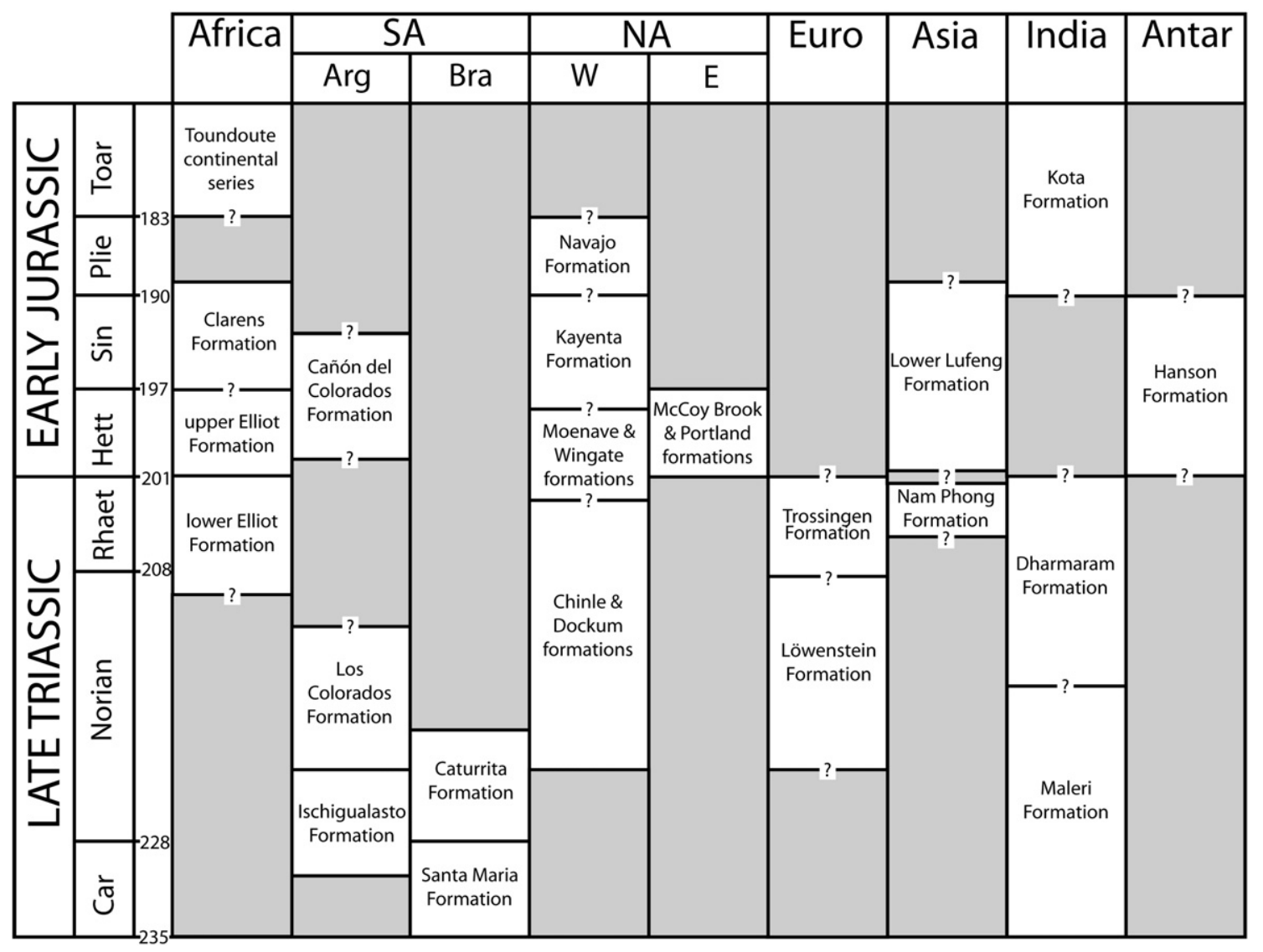

Fig. 4. A generalized geological correlation chart for the major Late Triassic and Early Jurassic dinosaur-bearing formations across the globe. The Triassic timescale at left is a modified version of Walker and Geissman (2009), with a longer Rhaetian following Muttoni et al. (2010). Note that most boundaries between formations, as well as global correlations, are imprecise due to many reasons discussed in the text. This is designed to provide a coarse guide of important early dinosaur faunas, not a precise correlation chart. Abbreviations: Arg = Argentina; Bra = Brazil; E = Eastern North America; $\mathrm{W}=$ Western North America. Chart delineated by Randall Irmis and Sterling Nesbitt. 
whose age is based solely on vertebrate biostratigraphy. The slightly younger Middle Triassic Los Chañares Formation of northwestern Argentina has been better sampled, and has yielded several basal dinosauromorph taxa, including at least one lagerpetid and silesaurid (Nesbitt et al., 2010) (Fig. 4). These strata are dated as Ladinian based on vertebrate biostratigraphy and the age of overlying strata (e.g., Rogers et al., 1993; Bonaparte, 1997; Rogers et al., 2001; Langer et al., 2007a). The oldest well-dated dinosaur-bearing assemblage is from the lower Ischigualasto Formation in northwestern Argentina. Rogers et al. (1993) reported a ${ }^{40} \mathrm{Ar} /{ }^{39} \mathrm{Ar}$ radioisotopic age of $227.8 \pm .3 \mathrm{Ma}$ from the lower portion of the formation. This age was recently revised to 231.4 Ma by Furin et al. (2006) to account for re-calibration of the age standard used in the original analysis as well as the bias in the $\mathrm{Ar} /$ Ar system that systematically yields ages $\sim 1 \%$ too young (e.g., Min et al., 2000; Mundil et al., 2006; Kuiper et al., 2008). Unpublished ${ }^{40} \mathrm{Ar} /$ ${ }^{39} \mathrm{Ar}$ ages indicate the top of the Ischigualasto Formation is between 223 and $220 \mathrm{Ma}$ (Shipman, 2004). Taken together, these data indicate that the formation spans the Carnian-Norian boundary. Therefore, the oldest dinosaurs from the Ischigualasto Formation are late Carnian in age, not early Carnian as previously reported (e.g., Rogers et al., 1993; Martinez and Alcober, 2009), and some Ischigualasto dinosaurs, notably Pisanosaurus, may be Norian in age (Irmis et al., 2007b; Langer et al., 2010).

Early dinosaur-bearing strata from southern Brazil are probably of similar age based on correlations to the Ischigualasto Formation using vertebrate biostratigraphy (e.g., Schultz et al., 2000; Langer, 2005; Langer et al., 2007a). The Chinle Formation of the Colorado Plateau in western North America is traditionally considered late CarnianNorian in age (e.g., Litwin et al., 1991; Lucas, 1998), but new U-Pb radioisotopic age constraints indicate that even the oldest fossiliferous strata are Norian in age (Riggs et al., 2003; Irmis and Mundil, 2008; Mundil et al., 2008). Footprint assemblages from the Newark Supergroup of eastern North America (e.g., Olsen et al., 2002) are tied to a high-resolution magnetostratigraphic record that is calibrated using palynomorph biostratigraphy (Kent and Olsen, 1999). Most other classic early dinosaur assemblages from the Late Triassic are dated primarily using biostratigraphic methods (conchostrachans, palynomorphs, vertebrates). These biochronologies have yet to be comprehensively calibrated with radioisotopic ages, so correlations to marine stages or the numerical Late Triassic timescale should be approached with caution.

\subsection{The paleoenvironment of early dinosaurs}

Global general circulation models for the Late Triassic Period predict warm and seasonal climates for most of Pangaea (Fig. 5). Lower latitude areas of Pangaea experienced summer temperatures above $35^{\circ} \mathrm{C}$, with slightly cooler winter temperatures. In contrast, high-latitude areas were warm during the summer $\left(>20^{\circ} \mathrm{C}\right)$, but near or below freezing during the winter (Sellwood and Valdes, 2006). These models predict very low levels of annual precipitation for lowlatitude Pangaea. These areas predominantly experienced summerwet precipitation (Sellwood and Valdes, 2006), though some midlatitude areas were arid throughout the year. The poles are assumed to have experienced cool temperate conditions (Sellwood and Valdes, 2006: fig. 2b).

Global syntheses suggest that there was a long-term decrease in atmospheric oxygen during the Late Triassic, but there is considerable disagreement about the duration and intensity of this event (e.g., Bergman et al., 2004; Berner, 2006; Algeo and Ingall, 2007). These data also suggest major fluctuations in atmospheric $\mathrm{CO}_{2}$ during the early Mesozoic (e.g., Berner, 2006). The general interpretation of these data is an increase in temperature and aridity through the Triassic, which is consistent with the general circulation model data. One complicating factor is that Laurasia moved progressively northward during the Late Triassic (Kent and Tauxe, 2005), but this would also explain an increase in aridity and seasonality as the landmass moved out of the tropics.

Previous authors have suggested linkages between climate change through the Triassic and terrestrial vertebrate evolution (e.g., Robinson, 1971; Tucker and Benton, 1982; Benton, 1983; Simms and Ruffell, 1990a). There was an overall change through three major

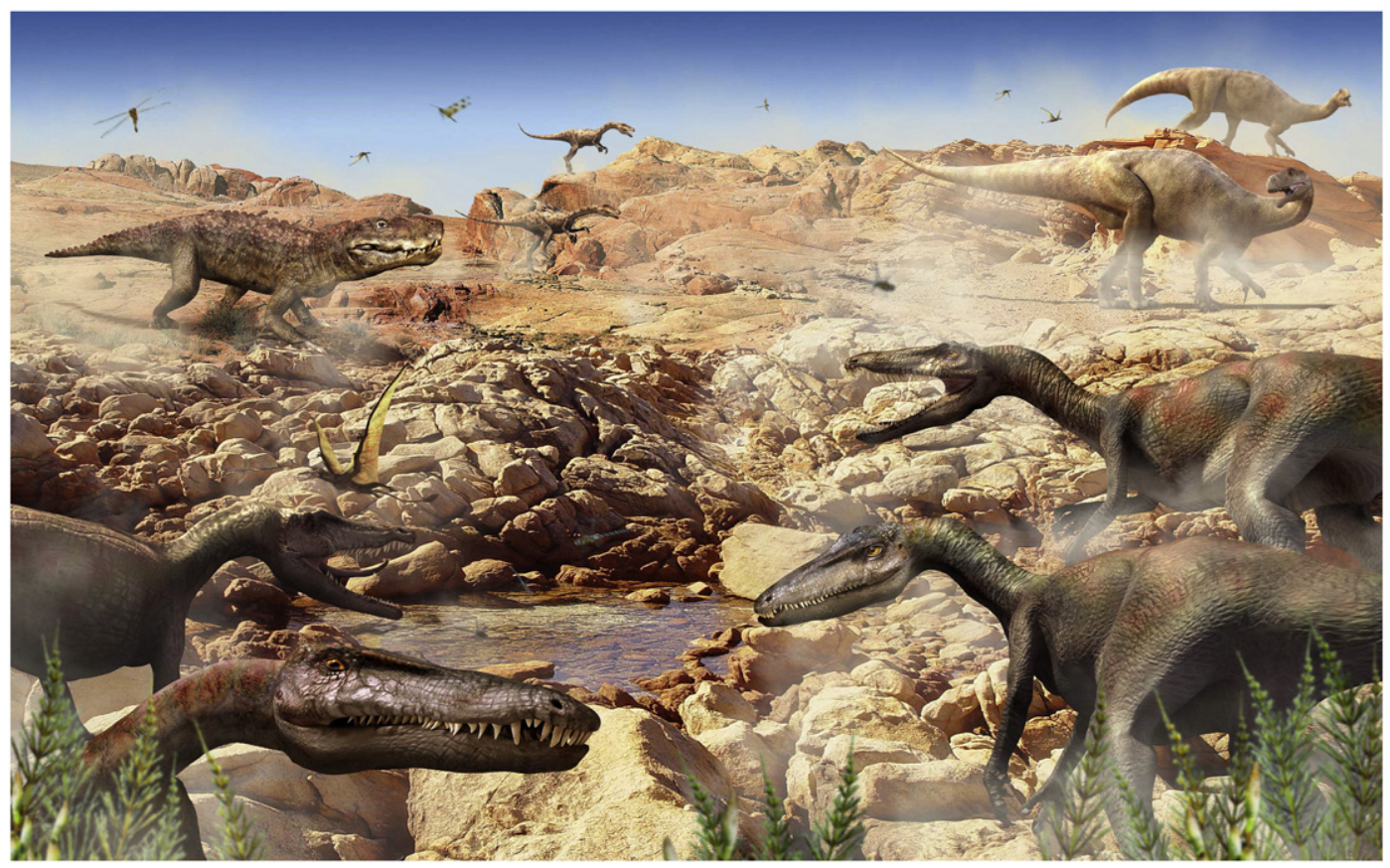

Fig. 5. A generalized reconstructed scene from the Late Triassic (Norian) of central Pangea, a dry and arid environment inhabited by the earliest dinosaurs and other archosaurs. A herd of the primitive theropod dinosaur Coelophysis congregates near a watering hole in the foreground. In the background a duo of Coelophysis stalks two herbivorous prosauropod dinosaurs, while a giant rauisuchian (quadrupedal crurotarsan predator) lurks in the distance and primitive pterosaurs (flying reptiles) soar overhead. Scene reconstructed using CGI and taken from Brusatte (2008), Dinosaurs (Quercus Publishing, London). Note that this is an artistic interpretation of a hypothetical Late Triassic community, not a scientifically accurate portrayal of a specific fossil assemblage. 
facies associations indicating increasing aridity. Deposition of typical red bed sediments in which tetrapods were preserved began in the Early Triassic of Gondwana and Russia. These beds are dominated by fluvio-lacustrine sandstones and mudrocks with coal seams and abundant plant material, indicating a mild and moist temperate regime. Middle Triassic and "Carnian" tetrapod sites worldwide are dominated by sediments indicating warm to hot climates, with variable humid and dry annual cycles, represented by fluviolacustrine sandstones and mudrocks with rare coals, some red beds and calcretes, occasional gypsum, and common plant fossils. The final facies association, fluvio-lacustrine red beds with calcrete, playa mudrocks, gypsum and halite deposits and aeolian sandstones, is seen in Norian to Early Jurassic successions in Gondwana, North America, and Europe. These units indicate hot sub-tropical arid/semi-arid climates with rare or erratic rainfall-essentially deserts, with alluvial fans and ephemeral streams, sand seas, playas, sabkhas and salt lakes.

The transition from the Carnian to Norian, or early to middle Norian if the successions are re-dated (see above), was also marked by a substantial shift from pluvial to arid conditions throughout the Tethyan realm (Simms and Ruffell, 1990a), and these major climatic changes may have been associated with floral changes from a predominantly Dicroidium-dominated flora in Gondwana to one based around arid-adapted conifers. Such climatic and floral changes might have precipitated extinctions of herbivorous rhynchosaurs and dicynodonts. These changes may coincide with the independently documented Reingraben Turnover/Raibl Event, which was a major restructuring of marine ecosystems during the middle and late Carnian (Furin et al., 2006; Stanley, 2006; Hornung et al., 2007). These consistent changes to more arid conditions could either be caused by global climate change, movement of continents through different climatic zones (e.g., Kent and Tauxe, 2005), or a combination of both factors.

Finally, a variety of evidence indicates severe environmental stress on land and in the ocean at the Triassic-Jurassic boundary, with a sharp increase in atmospheric $\mathrm{CO}_{2}$ levels (e.g., Smith and Kitching, 1997; McElwain et al., 1999; Cohen and Coe, 2007; Michalík et al., 2007; Hautmann et al., 2008; Whiteside et al., 2010). These environmental changes may have been associated with a mass extinction near the Triassic-Jurassic boundary, which is recognized as one of the "big five" mass extinctions in earth history (e.g., Raup, 1986; Benton, 1995). New work using records of compound-specific stable carbon isotopes from the Triassic-Jurassic boundary interval of the Newark Supergroup in eastern North America indicates that the eruption of flood basalts caused a massive input of greenhouse gases into the atmosphere, and that the release of this greenhouse gas and the earliest basalt flows are synchronous with biotic extinctions both on land and in the ocean (Whiteside et al., 2010). These data are the strongest evidence yet indicating flood volcanism caused the endTriassic mass extinction.

Aside from this general information on Triassic and Jurassic climate and environments, published paleoenvironmental proxy data for specific early dinosaur-bearing strata are limited (Fig. 5). Sedimentological, geochemical, and paleobotanical evidence indicates that the earliest known dinosaurs from the upper Carnian Ischigualasto Formation of Argentina lived in a dry seasonal climate that later fluctuated with wetter conditions during the early Norian (Moore, 2002; Shipman, 2004; Tabor et al., 2004, 2006; Colombi and Parrish, 2008; Currie et al., 2009). Multi-proxy evidence from the Norian Chinle Formation of western North America indicates that it was deposited under humid, wet, sub-tropical conditions during the early Norian, but that the paleoenvironment gradually became drier and more seasonal during the later Norian (e.g., Dubiel et al., 1991; Parrish, 1993; Dubiel, 1994; Prochnow et al., 2006), consistent with the northward drift of Laurasia (Kent and Tauxe, 2005). Data from northern New Mexico indicate that during the late Norian to Rhaetian the environment was semi-arid to arid, with moderate to severe fluctuations in a variety of environmental parameters (Cleveland et al., 2008a,b; Dunlavey et al., 2009).

\subsection{Early dinosaur-bearing formations}

Early dinosaurs are distributed across Pangaea. Footprints and body fossils are known from several sedimentary basins in Argentina, most notably the Ischigualasto and Los Colorados formations of the Ischigualasto-Villa Union Basin in northwestern Argentina (e.g., Rogers et al., 1993; Bonaparte, 1997; Zerfass et al., 2004). Similarly aged strata (Santa Maria and Caturrita formations) in southern Brazil preserve an extensive tetrapod assemblage, including basal dinosauromorphs, basal saurischians, and early sauropodomorphs (e.g., Langer et al., 2007a). Late Norian to Early Jurassic rocks of the Stormberg Group (primarily the lower and upper Elliot Formation) in southern Africa preserve diverse assemblages that are dominated by sauropodomorph dinosaurs (e.g., Olsen and Galton, 1984; Knoll, 2004, 2005). Basal saurischians and sauropodomorphs are known from Late Triassic sediments in the Pranhita-Godavari Valley in India (e.g., Kutty et al., 2007). Late Triassic dinosaurs are unknown from Madagascar, Antarctica, and Australia, but the Early Jurassic Hanson Formation in Antarctica preserves theropod and sauropodomorph dinosaurs (Smith and Pol, 2007; Smith et al., 2007).

Extensive early dinosaur assemblages are also known from Laurasia. In western North America, basal dinosauromorphs, basal saurischians, and theropods are known from the Norian Chinle Formation and Dockum Group. Overlying Early Jurassic strata preserve a diverse assemblage of ornithischians, sauropodomorphs, and theropods in the Glen Canyon Group (e.g., Tykoski, 2005). The dinosaur record of eastern North America is primarily documented by footprints, with extensive late Carnian, Norian, and Rhaetian dinosauromorph assemblages from the Newark Supergroup (Olsen and Huber, 1998; Olsen et al., 2002), but it also includes Early Jurassic body fossils of the sauropodomorph Anchisaurus (e.g., Yates, 2004) and theropods (Talbot, 1911; Colbert and Baird, 1958). Norian and Rhaetian terrestrial strata from the Germanic Basin in Europe are dominated by basal sauropodomorphs (e.g., Yates, 2003b), but theropods are also present (Schoch and Wild, 1999). Similar assemblages have been reported from Greenland (Jenkins et al., 1994), and Dzik et al. (2008) recently reported theropod dinosaurs from the latest Triassic of Poland. Poorly dated fissure fills from western Europe record the presence of sauropodomorphs and possible theropods (e.g., Benton et al., 2000; Yates, 2003a); these are generally thought to be latest Triassic to Early Jurassic in age (Whiteside and Marshall, 2008). Dinosaurs are conspicuously absent from the Late Triassic of Asia, but an extensive sauropodomorph-dominated assemblage is known from the Lower Jurassic Lufeng Formation of Yunnan, China; this assemblage also includes rare ornithischians and theropods (e.g., Luo and $\mathrm{Wu}, 1994)$.

\section{Dinosaurs of the Late Triassic and Early Jurassic}

The following is a summary of the evolution and distribution of the major dinosaur subgroups during the Late Triassic and Early Jurassic (Fig. 3). A complete list of all valid dinosaur taxa known from this time span is given in Table 1. A framework cladogram showing the general phylogenetic relationships of early dinosaurs is given in Fig. 6.

\subsection{Ambiguous taxa: Eoraptor and Herrerasauridae}

Two taxa from the Ischigualasto Formation of Argentina, Herrerasaurus ischigualastensis (Fig. 3A) and Eoraptor lunensis, are represented by some of the most complete specimens of any early dinosaur, yet their phylogenetic position has been the source of vigorous debate. Emerging evidence, most notably a revised understanding of dinosaur character evolution buoyed by the discovery of the nearly 
Table 1

A list of Late Triassic and Early Jurassic dinosaur species, divided into the three major dinosaur subgroups (Theropoda, Ornithischia, Sauropodomorpha), as well as six taxa that are clearly dinosaurs but of uncertain position. All named and valid species are included, as well as a number of unnamed specimens that are likely diagnostic and represent valid species-level taxa.

\begin{tabular}{|c|c|c|c|c|}
\hline Taxon & Geographic area & Formation & Age & References \\
\hline \multicolumn{5}{|l|}{ Dinosauria incertae sedis } \\
\hline Chindesaurus bryansmalli & Southwestern USA & Chinle and Tecovas formations & Middle Norian & Long and Murry (1995) \\
\hline Herrerasaurus ischigualastensis & Argentina & Ischigualasto Formation & Late Carnian-early Norian & $\begin{array}{l}\text { Reig (1963); Sereno and Novas (1992); Sereno } \\
\text { (1993); Sereno and Novas (1994); Novas (1993) }\end{array}$ \\
\hline Eoraptor lunensis & Argentina & Ischigualasto Formation & Late Carnian-early Norian & Sereno et al. (1993) \\
\hline Staurikosaurus pricei & Brazil & Santa Maria Formation & Late Carnian-early Norian & Colbert (1970); Bittencourt and Kellner (2009) \\
\hline Guaibasaurus candelariensis & Brazil & Caturrita Formation & Norian & Bonaparte et al. (1999); Langer and Benton (2006) \\
\hline Agnosphitys cromhallensis & United Kingdom & Cromhall Quarry & Norian-Rhaetian & Fraser et al. (2002) \\
\hline \multicolumn{5}{|l|}{ Sauropodomorpha } \\
\hline Anchisaurus polyzelus & Connecticut, USA & Portland Formation & Pliensbachian-Toarcian & $\begin{array}{l}\text { Hitchcock (1865); Galton (1976); } \\
\text { Yates (2004); Fedak and Galton (2007) }\end{array}$ \\
\hline "Massospondylus sp." & Arizona, USA & Kayenta Formation & Hettangian-Sinemurian & Attridge et al. (1985) \\
\hline $\begin{array}{l}\text { Unnamed basal } \\
\text { sauropodomorph(s) }\end{array}$ & Arizona, USA & Navajo Formation & Pliensbachian-Toarcian & $\begin{array}{l}\text { Brady }(1935,1936) \text {; Galton }(1971,1976) \text {; } \\
\text { Yates (2004); Irmis (2005) }\end{array}$ \\
\hline Seitaad ruessi & Utah, USA & Navajo Formation & Pliensbachian-Toarcian & Sertich and Loewen (2010) \\
\hline Panphagia protos & Argentina & Ischigualasto Formation & Late Carnian-early Norian & Martinez and Alcober (2009) \\
\hline Coloradosaurus brevis & Argentina & Los Colorados Formation & Norian-?Rhaetian & Bonaparte (1978) \\
\hline Lessemsaurus sauropoides & Argentina & Los Colorados Formation & Norian-?Rhaetian & Bonaparte (1999); Pol and Powell (2007a) \\
\hline Riojasaurus incertus & Argentina & Los Colorados Formation & Norian-?Rhaetian & Bonaparte (1969) \\
\hline Mussaurus patagonicus & Argentina & Laguna Colorada Formation & ?Norian & $\begin{array}{l}\text { Bonaparte and Vince (1979); } \\
\text { Pol and Powell (2007b) }\end{array}$ \\
\hline Adeopapposaurus mognai & Argentina & Cañón del Colorado Formation & Early Jurassic & Martínez (2009) \\
\hline Saturnalia tupiniquim & Brazil & Santa Maria Formation & Carnian-early Norian & Langer et al., 1999; Langer (2003) \\
\hline Unaysaurus tolentinoi & Brazil & Caturrita Formation & Norian & Leal et al. (2004) \\
\hline Glacialisaurus hammeri & Antarctica & Hanson Formation & $\begin{array}{l}\text { Sinemurian- } \\
\text { Pliensbachian }\end{array}$ & Smith and Pol (2007) \\
\hline Melanorosaurus readi & South Africa & Lower Elliot Formation & Norian & Haughton (1924); Yates (2007a) \\
\hline Eucnemesaurus fortis & South Africa & Lower Elliot Formation & Norian & Haughton (1924); Yates (2007b) \\
\hline Plateosauravus cullingworthi & South Africa & Lower Elliot Formation & Norian & Haughton (1924) \\
\hline Blikanasaurus cromptoni & South Africa & Lower Elliot Formation & Norian & Galton and van Heerden (1985) \\
\hline Antetonitrus ingenipes & South Africa & Lower Elliot Formation & Norian & Yates and Kitching (2003) \\
\hline Massospondylus carinatus & South Africa/Lesotho & $\begin{array}{l}\text { Upper Elliot Formation } \\
\text { and Clarens Formation }\end{array}$ & Hettangian-Pliensbachian & Owen (1854) \\
\hline Massospondylus kaalae & South Africa & Upper Elliot Formation & Hettangian-Sinemurian & Barrett $(2004,2009 b)$ \\
\hline Ignavusaurus rachelis & Lesotho & Upper Elliot Formation & Hettangian-Sinemurian & Knoll (in press) \\
\hline Aardonyx celestae & South Africa & Upper Elliot Formation & Hettangian-Sinemurian & Yates et al. (2010) \\
\hline Vulcanodon karibaensis & Zimbabwe & Vulcanodon Beds & ?Hettangian & Raath (1972) \\
\hline Tazoudasaurus naimi & Morocco & Toundoute Continental Series & Toarcian & Allain et al. (2004) \\
\hline $\begin{array}{l}\text { Lamplughsaura } \\
\text { dharmaramensis }\end{array}$ & India & Upper Dharmaram Formation & ?Sinemurian & Kutty et al. (2007) \\
\hline Pradhania gracilis & India & Upper Dharmaram Formation & ?Sinemurian & Kutty et al. (2007) \\
\hline Barapasaurus tagorei & India & Kota Formation & Hettangian-Pliensbachian & Jain et al. (1975) \\
\hline Kotasaurus yamanpalliensis & India & Kota Formation & Hettangian-Pliensbachian & Yadagiri $(1988,2001)$ \\
\hline Plateosaurus gracilis & Germany & Lowenstein Formation & Early Norian & von Huene (1908); Yates (2003b) \\
\hline Efraasia minor & Germany & Lowenstein Formation & Mid-late Norian & von Huene (1932); Galton (1973); Yates (2003b) \\
\hline Plateosaurus engelhardti & Germany & $\begin{array}{l}\text { Lowenstein Formation and } \\
\text { Trossingen Formation }\end{array}$ & Mid Norian-Rhaetian & von Meyer (1837); Yates (2003b) \\
\hline Plateosaurus ingens & Germany & Trossingen Formation & Rhaetian & Rutimeyer (1856); Galton (2001) \\
\hline Ruehleia bedheimensis & Germany & Trossingen Formation & Rhaetian & Galton (2001) \\
\hline Thecodontosaurus antiquus & United Kingdom & Magnesian Conglomerate & Norian-?Rhaetian & Riley and Stutchbury (1836) \\
\hline Pantydraco caducus & United Kingdom & Pant-y-ffynnon Quarry & Norian-?Rhaetian & Yates (2003a); Galton et al. (2007) \\
\hline Lufengosaurus huenei & China & Lower Lufeng Series & Early Jurassic & Young (1941); Barrett et al. (2005) \\
\hline Jingshanosaurus xinwaensis & China & Lower Lufeng Series & Early Jurassic & Zhang and Yang (1994) \\
\hline Yunnanosaurus huangi & China & Lower Lufeng Series & Early Jurassic & Young (1942): Barrett et al. (2007) \\
\hline Yimenosaurus youngi & China & Fengjiahe Formation & Pliensbachian or Toarcian & Bai et al. (1990) \\
\hline $\begin{array}{l}\text { Chinshakiangosaurus } \\
\text { chunghoensis }\end{array}$ & China & Fengjiahe Formation & Pliensbachian or Toarcian & Upchurch et al (2007b) \\
\hline Gongxianosaurus shibeiensis & China & Ziliujing Formation & Early Jurassic & He et al. (1998) \\
\hline Isanosaurus attavipachi & Thailand & Nam Phong Formation & Late Norian-Rhaetian & Buffetaut et al. (2000) \\
\hline \multicolumn{5}{|l|}{ Theropoda } \\
\hline Tawa hallae & New Mexico, USA & Chinle Formation & Norian-?Rhaetian & Nesbitt et al. (2009b) \\
\hline Coelophysis bauri & New Mexico, USA & Chinle Formation & Norian-?Rhaetian & Cope (1889),Colbert (1989) \\
\hline Unnamed coelophysoid & New Mexico, USA & Chinle Formation & Norian & Heckert et al. $(2000,2003)$ \\
\hline Gojirasaurus quayi & New Mexico, USA & Bull Canyon Formation & Norian & Carpenter (1997); Nesbitt et al (2007) \\
\hline $\begin{array}{l}\text { Coelophysoidea indet. } \\
\text { ("Camposaurus") }\end{array}$ & Arizona, USA & Chinle Formation & Early Norian & $\begin{array}{l}\text { Long and Murry (1995); Hunt et al (1998); } \\
\text { Nesbitt et al (2007) }\end{array}$ \\
\hline Dilophosaurus wetherilli & Arizona, USA & Kayenta Formation & Hettangian-Sinemurian & Welles $(1954,1970,1984)$ \\
\hline "Syntarsus" kayentakatae & Arizona, USA & Kayenta Formation & Hettangian-Sinemurian & Rowe (1989) \\
\hline $\begin{array}{l}\text { Unnamed theropod } \\
\text { ("Shake-N-Bake Theropod") }\end{array}$ & Arizona, USA & Kayenta Formation & Hettangian-Sinemurian & Tykoski (1997, 2005); Tykoski and Rowe (2004) \\
\hline Segisaurus halli & Arizona, USA & Navajo Formation & Pliensbachian-Toarcian & Camp (1936); Carrano et al. (2005) \\
\hline Podokesaurus holyokensis & Massachusetts, USA & ?Portland Formation & Pliensbachian-Toarcian & Talbot (1911) \\
\hline Zupayaurus rougieri & Argentina & Los Colorados Formation & Norian-?Rhaetian & $\begin{array}{l}\text { Arcucci and Coria (2003); Ezcurra (2007); } \\
\text { Ezcurra and Novas (2007) }\end{array}$ \\
\hline
\end{tabular}


Table 1 (continued)

\begin{tabular}{|c|c|c|c|c|}
\hline Taxon & Geographic area & Formation & Age & References \\
\hline \multicolumn{5}{|l|}{ Theropoda } \\
\hline Cryolophosaurus ellioti & Antarctica & Hanson Formation & $\begin{array}{l}\text { Sinemurian- } \\
\text { Pliensbachian }\end{array}$ & Hammer and Hickerson (1994); Smith et al. (2007) \\
\hline Coelophysis rhodesiensis & $\begin{array}{l}\text { Zimbabwe, } \\
\text { South Africa }\end{array}$ & $\begin{array}{l}\text { Forest Sandstone and } \\
\text { upper Elliot formations }\end{array}$ & Hettangian-?Sinemurian & Raath (1969) \\
\hline Dracovenator regenti & South Africa & Upper Elliot Formation & Hettangian-Sinemurian & Yates (2005) \\
\hline Berberosaurus liassicus & Morocco & Toundoute continental series & Pliensbachian-Toarcian & Allain et al. (2007) \\
\hline Unnamed theropod & Morocco & Wazzant Formation & Toarcian & Jenny et al. (1980); Taquet (1984) \\
\hline Procompsognathus triassicus & Germany & Lowenstein Formation & Norian & $\begin{array}{l}\text { Fraas, 1913; Sereno and Wild (1992); } \\
\text { Rauhut and Hungerbühler, 2000; Knoll (2008) }\end{array}$ \\
\hline Liliensternus liliensterni & Germany & Knollenmergel & Norian & $\begin{array}{l}\text { von Huene (1934); Welles (1984); Rauhut and } \\
\text { Hungerbühler, 2000; Rauhut (2003) }\end{array}$ \\
\hline Lophostropheus airelensis & France & Moon-Airel Formation & ?Rhaetian-Hettangian & $\begin{array}{l}\text { Cuny and Galton (1993); Rauhut and } \\
\text { Hungerbühler (2000); Ezcurra and Cuny (2007) }\end{array}$ \\
\hline Unnamed theropod & Poland & Lipie Śląskie clay-pit & ?Norian-Rhaetian & Dzik et al. (2008) \\
\hline Sarcosaurus andrewsi & England & Lower Lias & Late Sinemurian & Andrews (1921); Carrano and Sampson (2008) \\
\hline Unnamed theropod & England & Lower Lias & Late Sinemurian & Newman (1968); Carrano and Sampson $(2004,2008)$ \\
\hline ?Dilophosaurus sinensis & China & Lower Lufeng Series & Early Jurassic & Hu (1993) \\
\hline Eshanosaurus deguchiianus & China & Lower Lufeng Series & Hettangian & Zhao and Xu (1998); Xu et al. (2001) \\
\hline \multicolumn{5}{|l|}{ Ornithischia } \\
\hline Scutellosaurus lawleri & Arizona, USA & Kayenta Formation & Hettangian-Sinemurian & Colbert (1981); Rosenbaum and Padian (2000) \\
\hline $\begin{array}{l}\text { Unnamed thyreophoran } \\
\text { ("Scelidosaurus sp.") }\end{array}$ & Arizona, USA & Kayenta Formation & Hettangian-Sinemurian & Padian (1989); Tykoski (2005) \\
\hline Unnamed heterodontosaurid & Arizona, USA & Kayenta Formation & Hettangian-Sinemurian & Attridge et al. (1985) \\
\hline Pisanosaurus mertii & La Rioja, Argentina & Ischigualasto Formation & Early Norian & $\begin{array}{l}\text { Casamiquela (1967); Bonaparte (1976); } \\
\text { Sereno (1991a,b); Irmis et al. (2007b) }\end{array}$ \\
\hline Unnamed heterodontosaurid & Santa Cruz, Argentina & Laguna Colorada Formation & ?Norian & Báez and Marsicano (2001) \\
\hline Unnamed ornithischian & Venezuela & La Quinta Formation & Early or Middle Jurassic & Barrett et al. (2008) \\
\hline Eocursor parvus & South Africa & Lower Elliot Formation & Norian & Butler et al. (2007) \\
\hline Lycorhinus angustidens & South Africa & Upper Elliot Formation & Hettangian-Sinemurian & Haughton (1924); Hopson (1975); Gow (1990) \\
\hline Abrictosaurus consors & Lesotho & Upper Elliot Formation & Hettangian-Sinemurian & Thulborn (1970b); Hopson (1975) \\
\hline BMNH A100 & Lesotho & Upper Elliot Formation & Hettangian-Sinemurian & $\begin{array}{l}\text { Thulborn (1970b); Hopson (1975); } \\
\text { Butler et al. (2008a,b) }\end{array}$ \\
\hline Heterodontosaurus tucki & South Africa & $\begin{array}{l}\text { Upper Elliot Formation and } \\
\text { Clarens Formation }\end{array}$ & Hettangian-Pliensbachian & $\begin{array}{l}\text { Crompton and Charig (1962); Santa Luca (1980); } \\
\text { Butler et al. (2008a,b) }\end{array}$ \\
\hline Lesothosaurus diagnosticus & South Africa/Lesotho & Upper Elliot Formation & Hettangian-Sinemurian & $\begin{array}{l}\text { Thulborn (1970a, 1971, 1972); Galton (1978); } \\
\text { Sereno (1991b); Butler (2005) }\end{array}$ \\
\hline Stormbergia dangershoeki & South Africa/Lesotho & Upper Elliot Formation & Hettangian-Sinemurian & Butler (2005) \\
\hline Emausaurus ernsti & Mecklenberg, Germany & Unnamed unit & Early Toarcian & Haubold (1990) \\
\hline Scelidosaurus harrisonii & Dorset, England & Lower Lias & Late Sinemurian & Owen $(1861,1863)$ \\
\hline Bienosaurus lufengensis & Yunnan, PR China & $\begin{array}{l}\text { Dark Red Beds of the } \\
\text { Lower Lufeng }\end{array}$ & Sinemurian & Dong (2001) \\
\hline Tatisaurus oehleri & Yunnan, PR China & $\begin{array}{l}\text { Dark Red Beds of the } \\
\text { Lower Lufeng }\end{array}$ & Sinemurian & Simmons (1965); Norman et al. (2007) \\
\hline
\end{tabular}

complete basal theropod Tawa (Nesbitt et al., 2009b), suggests that both taxa are true theropods, as originally argued by Sereno and colleagues in the early 1990s (see below). However, given the continued limited character support for this phylogenetic placement (e.g., Nesbitt et al., 2009b: SOM), it is probable that the relationships of Herrerasaurus and Eoraptor will remain contentious.

Herrerasaurus is a bipedal carnivore that reached lengths of up to $4 \mathrm{~m}$. It was originally hypothesized to be the immediate sister taxon (closest relative) to Dinosauria (as defined in this paper) (Gauthier, 1986; Brinkman and Sues, 1987; Novas, 1992). Some subsequent authors regarded Herrerasaurus as a true dinosaur, but of uncertain phylogenetic position (Novas, 1989). The discovery of more complete specimens in the late 1980s demonstrated that Herrerasaurus is a true dinosaur, but also gave rise to two opposing viewpoints on its affinities: some authors regard it as a stem saurischian outside of the theropod + sauropodomorph group (Langer, 2004; Langer and Benton, 2006; Irmis et al., 2007a), whereas others argue that it is a basal member of the theropod lineage (Sereno et al., 1993; Novas, 1996; Sereno, 1997, 1999; Rauhut, 2003; Sereno, 2007a; Nesbitt et al., 2009b). This debate is currently one of the most important unresolved questions regarding early dinosaur phylogeny and evolution.

Herrerasaurus is usually grouped with another carnivorous dinosaur, Staurikosaurus pricei, within a subclade of early dinosaurs called the Herrerasauridae, which is supported by a number of unique derived characters (Langer and Benton, 2006; Nesbitt et al., 2009b). Staurikosaurus is known from the Santa Maria sequence in Brazil, which is approximately the same age as the Ischigualasto Formation (Langer, 2005; Langer et al., 2007a). Staurikosaurus is represented by a single partial skeleton, which is substantially less complete than specimens of Herrerasaurus, including the mandible, most of the vertebral column, pelvic girdle, and partial hindlimbs (Colbert, 1970). A comprehensive redescription of Staurikosaurus has recently been undertaken, and should help clarify its anatomy and phylogenetic position (Bittencourt and Kellner, 2009).

The phylogenetic position of the much smaller Eoraptor, a predator or omnivore that reached lengths of $1-2 \mathrm{~m}$, is equally controversial. Although all phylogenetic analyses have placed Eoraptor as a member of the saurischian lineage, there is debate over whether it is a true theropod or a more primitive stem saurischian dinosaur outside of the theropod + sauropodomorph clade. Sereno et al. (1993) found Eoraptor as the most primitive theropod, outside of a more derived group that includes Herrerasaurus and all other theropods; this result was also found by Novas (1996), Sereno (1999), and Rauhut (2003). However, Langer and Benton (2006) found Eoraptor as more derived than Herrerasaurus but outside of the theropod + sauropodomorph clade. Thus, neither Eoraptor nor Herrerasaurus is a true theropod in 


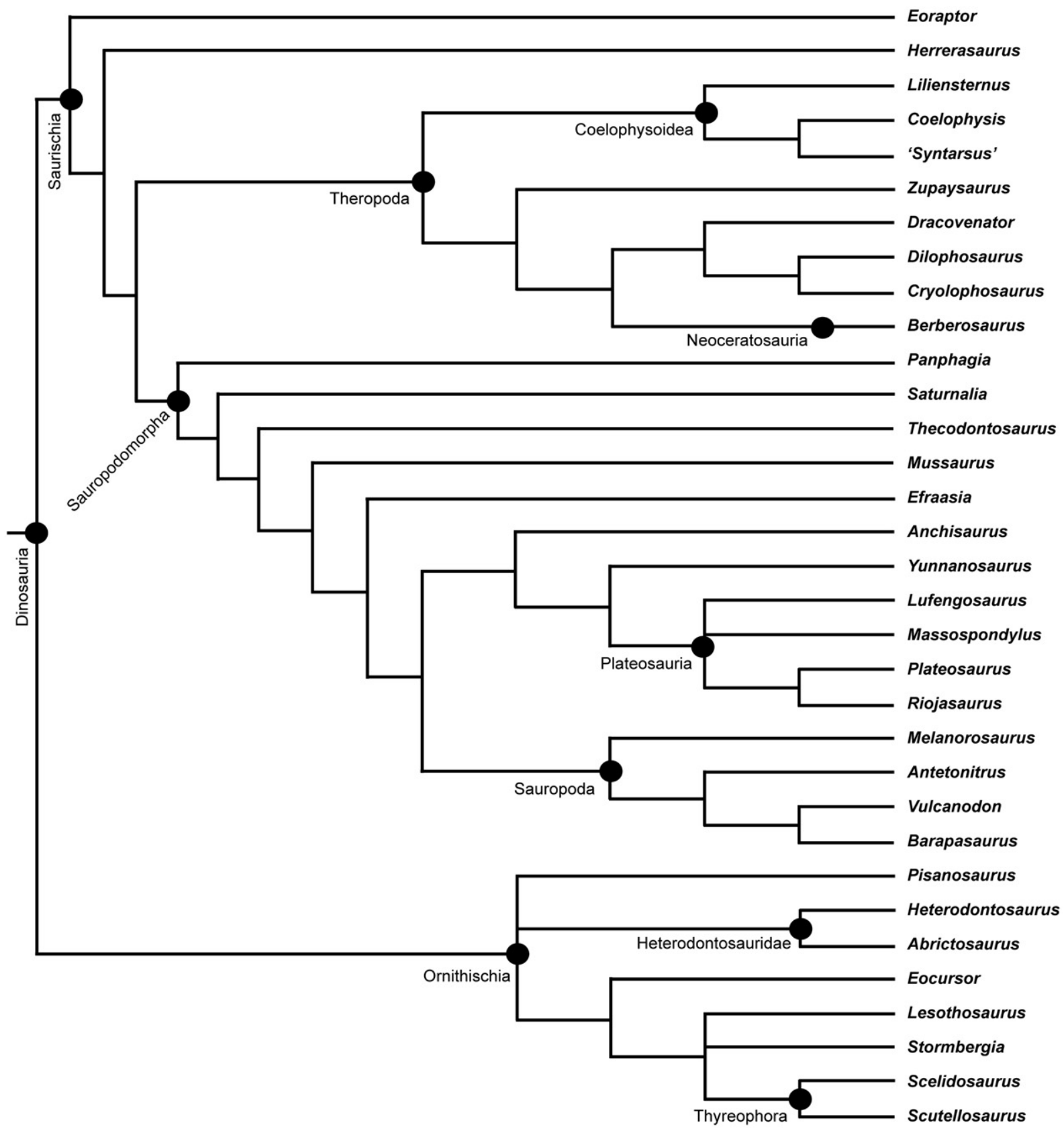

Fig. 6. A framework phylogeny (cladogram) of several of the most complete and important Triassic and Jurassic dinosaurs, with major clades denoted. This phylogeny does not result from a novel cladistic analysis, but is a summary cladogram that relies heavily on the analyses of Langer and Benton (2006) for overall dinosaur relationships, Smith et al. (2007) for theropod relationships, Butler et al. (2007) for ornithischian relationships, and Upchurch et al. (2007a) for sauropodomorph relationships. Please refer to the original cladistic analyses for further details. Cladogram delineated by Stephen Brusatte.

Langer and Benton's (2006) phylogeny. Most recently, Nesbitt et al. (2009b) recovered Eoraptor as a theropod, but more derived than Herrerasaurus. Much of this instability may relate to the fact that Eoraptor has yet to be fully described, though Langer (Langer and Benton, 2006: p. 311), Irmis (Irmis et al., 2007a,b), and Nesbitt (Nesbitt et al., 2009b) did examine the specimen first-hand.

Chindesaurus bryansmalli and Guaibasaurus candelariensis are two other enigmatic saurischian dinosaurs from the Late Triassic. Chindesaurus is from the Upper Triassic (Norian) Chinle Formation of Arizona (Long and Murry, 1995; Nesbitt et al., 2007). It reached lengths of 2-3 m, and is mainly known from the incomplete skeleton of the holotype specimen, which preserves a partial vertebral column, pelvis, and hindlimbs. Clearly a saurischian (Nesbitt et al., 2007), Chindesaurus has been included in the clade Herrerasauridae as a theropod (Long and
Murry, 1995; Hunt, 1996; Novas, 1996; Hunt et al., 1998; Nesbitt et al. $2009 \mathrm{~b}$ ) and as a stem saurischian outside of the theropod + sauropodomorph group (Langer, 2004; Irmis et al., 2007a). New material of Chindesaurus from the Hayden Quarry (Irmis et al., 2007a) may help clarify its ambiguous systematic position. Guaibasaurus, from the Caturrita Formation of southern Brazil, is known from three specimens that together preserve most of the skeleton, except for the skull (Bonaparte et al., 1999, 2007). Unfortunately, the articular ends of the bones are poorly preserved; thus, important character states of the femur, tibia, and ankle cannot be scored using available material. Guaibasaurus has been considered either the sister taxon to saurischians (Bonaparte et al., 1999, 2007) or a basal theropod (Langer, 2004; Langer and Benton, 2006; Yates, 2007a,b). Its phylogenetic position is still unresolved, but it can be confidently placed within Saurischia (Langer et al., 2007a,c). 
All of these controversial taxa share skeletal features with unequivocal carnivorous theropods, such as recurved and serrated teeth and elongate hands with recurved claws (Fig. 3A,B; Sereno et al., 1993; Sereno, 1999; Langer, 2004). Some authors suggest that these common features are homologous between all of these taxa and neotheropods (e.g., Sereno, 1999), whereas others consider such characteristics to be convergent, usually based on character optimization in a phylogenetic analysis (Langer, 2004; Langer and Benton, 2006). Nevertheless, the distribution and interpretation of some "predatory" features among basal dinosaurs is complicated. For example, one of the basal-most ornithischians, Heterodontosaurus (see below), has an elongated manus with clearly recurved claws, characters present in Herrerasaurus and unequivocal theropods. Thus, these features may simply represent the plesiomorphic condition for Dinosauria rather than derived specialization indicative of preferential relationship to theropods. These characters are also difficult to interpret phylogenetically. For example, the proposed homology of the intramandibular jaw joint that allows motion between bones of the lower jaw for Herrerasaurus and theropods is unclear, given that the joint is constructed differently in these two taxa (Sereno, 1999; Langer and Benton, 2006).

These character conflicts, along with many others, help explain why the phylogenetic positions of Eoraptor, Chindesaurus, Guaibasaurus, Herrerasaurus, and Staurikosaurus remain unresolved to date. As with many paleontological debates, it is likely that new fossil discoveries of these dinosaurs or closely related taxa will help resolve this puzzle. Indeed, the recent discovery of Tawa, a remarkably complete basal theropod, may prove instrumental, as its combination of primitive and derived characters help pull Herrerasaurus, Chindesaurus, Staurikosaurus, and Eoraptor into the theropod clade in the largest and most up-to-date phylogenetic analysis of basal dinosaurs yet published (Nesbitt et al., 2009b).

\subsection{Theropods}

\subsubsection{Late Triassic theropods - fossil record and distribution}

The first definitive theropods are known from the Norian. Previous records of Carnian theropods, such as the coelophysoid "Camposaurus" (Hunt et al., 1998), have been recently re-dated as Norian (Nesbitt et al., 2007; Irmis and Mundil, 2008). However, if Eoraptor, Herrerasaurus, or Staurikosaurus are basal members of Theropoda, as hypothesized by Sereno et al. (1993), Sereno (1999), Nesbitt et al. (2009b), and others, then this clade would extend into the Carnian. Regardless, the presence of Carnian sauropodomorphs-members of the sister taxon of Theropoda-imply that the theropod lineage also extends into the Carnian by virtue of its ghost lineage (e.g., Langer et al., 1999; Martinez and Alcober, 2009).

Theropods are generally rare in Late Triassic assemblages and exhibit low taxonomic diversity and a relatively restricted range of morphology compared to Early Jurassic members of the group. Most known definitive Late Triassic theropods may belong to a major clade called Coelophysoidea (e.g., Sereno, 1999; Carrano et al., 2002; Rauhut, 2003; Tykoski and Rowe, 2004; Carrano et al., 2005; Ezcurra and Novas, 2007; Smith et al., 2007). The most basal major clade of theropod dinosaurs, Coelophysoidea includes a range of mostly smallbodied predators such as Coelophysis, "Syntarsus," Liliensternus, Lophostropheus, Gojirasaurus, and Procompsognathus (Table 1). Several indeterminate coelophysoids are also known, and it is clear that this clade was geographically widespread during the Late Triassic and possibly abundant in some ecosystems (e.g., Ghost Ranch: Colbert, 1989). Recently, however, it has been proposed that Coelophysoidea, as traditionally considered, is a paraphyletic grade on the line to more derived theropods (Smith et al., 2007; Nesbitt et al., 2009b). It may be that Coelophysoidea is a restricted clade that only includes Coelophysis and close relatives (known as Coelophysidae), but this awaits further testing and corroboration. What is clear, however, is that the recently described Tawa from the Norian of New Mexico is more basal than taxa traditionally regarded as coelophysoids, and thus outside the clade Neotheropoda (Nesbitt et al., 2009b).

The puzzling Argentine theropod Zupaysaurus was initially described as the oldest tetanuran theropod (see below), but has been reinterpreted as a more basal taxon (e.g., Ezcurra, 2007). However, whether it falls within the coelophysoid clade or is outside of this clade and more closely related to tetanurans is a matter of debate (e.g., compare the phylogenies of Ezcurra and Novas (2007) with Smith et al. (2007) and Nesbitt et al. (2009b)). Definitive tetanuran and/or neoceratosaurian theropods are still unknown from the Late Triassic, and neither lineage can be confidently extended into this time using ghost lineages.

One final specimen deserves comment. Dzik et al. (2008) briefly described a number of well-preserved fossils from the latest Triassic (?Rhaetian) of Poland, which they interpreted as representing a large theropod dinosaur ( $\sim 3 \mathrm{~m}$ in length). They argued that this specimen extends the fossil record of large theropods, otherwise known from the Early-Middle Jurassic, into the Late Triassic. However, Triassic theropods of the same general size of the new Polish material are already known (Gojirasaurus, Liliensternus). Although two of us (SLB, $\mathrm{RJB}$ ) have examined the specimens, we await a full description of the material before commenting on its phylogenetic and evolutionary importance. Regardless of the affinities of these large specimens, there are unequivocal small theropod vertebrae (described as coelophysoids by Dzik et al., 2008) in the same quarry.

\subsubsection{Late Triassic theropods - paleobiology}

Most Late Triassic theropods were small-bodied and gracile. The familiar Coelophysis bauri, which reached an average length of about $2 \mathrm{~m}$ and a mass of 10-40 kg (Peczkis, 1994), is a useful general model for Late Triassic theropod size and morphology. However, the coelophysoid Liliensternus reached much larger body sizes, and may have approached about $6 \mathrm{~m}$ in length and up to $400 \mathrm{~kg}$ in mass (Peczkis, 1994). The same is also true of Gojirasaurus, which is estimated at $5.5 \mathrm{~m}$ in length (Carpenter, 1997). Truly colossal theropods, in the size range of Allosaurus ( $~ 8 \mathrm{~m}$ in length) and greater, are unknown from the Late Triassic.

Late Triassic coelophysoids, as well as Tawa, possessed the specializations seen in most predatory theropods. The skull was elongate, filled with an array of serrated and recurved teeth, and well constructed to withstand the high stresses of biting prey (Rayfield, 2005). The feet and hands were capped with sharp claws. The skeleton itself was light and gracile and the tail was long and stiff for balance, features that enabled speed and maneuverability. These theropods were most likely active predators. Coelophysis has long been described as a cannibal that fed on the remains of its own young (e.g., Colbert, 1989), but recent reinterpretation reveals that the supposed infant Coelophysis bones in the gut of one specimen belong to an early crocodylomorph (Nesbitt et al., 2006).

The spectacular fossil assemblage of Ghost Ranch, New Mexico, gives an unprecedented view of dinosaur community and population structure. This assemblage includes the remains of hundreds of Coelophysis individuals, ranging from small juveniles to adults (Colbert, 1989). Many skeletons are complete, articulated, and exceptionally well preserved, and are buried within abandoned channel deposits that indicate rapid burial after minor transport (Schwartz and Gillette, 1994). It is likely that this assemblage preserves a group of individuals that was overtaken by a rapid environmental crisis, such as a drought or flood (Colbert, 1989; Schwartz and Gillette, 1994). As such, it is one of the few sites in the Mesozoic fossil record where a potential theropod dinosaur community is well represented (Irmis, 2009; Rinehart et al., 2009).

Although hundreds of skeletons of Coelophysis are known from Ghost Ranch, theropods are generally rare components of other Late Triassic ecosystems (e.g., Rauhut and Hungerbühler, 2000; Nesbitt 
et al., 2007). Carnivorous theropods are much rarer (in an absolute faunal abundance sense) and less diverse (in a taxonomic sense) than contemporary carnivorous crurotarsans such as phytosaurs, ornithosuchids, and rauisuchians (e.g., Welles, 1986). This is borne out by Benton's (1983) compilation of absolute faunal abundance in Late Triassic fossil sites, although careful studies of crurotarsan taxonomic diversity have yet to be undertaken. Similarly, carnivorous theropods are much less morphologically disparate than carnivorous crurotarsans in the Late Triassic (Brusatte et al., 2008a,b).

\subsubsection{Early Jurassic theropods - fossil record and distribution}

Early Jurassic theropods are much more common, taxonomically diverse, and exhibit a greater range of morphologies than Late Triassic members of the group. Whereas only coelophysoids and similar taxamostly small-bodied and primitive theropods-were present in the Late Triassic, the Early Jurassic witnessed the evolution of more derived theropod clades characterized by larger body size and more disparate morphology. Most importantly, two major theropod clades, each of which would persist until the end of the Cretaceous, originated during the Early Jurassic.

The first of these clades, Ceratosauria (also called Neoceratosauria), would later give rise to the familiar Late Jurassic Ceratosaurus and the speciose Cretaceous clade Abelisauroidea (e.g... Tykoski and Rowe, 2004; Carrano and Sampson, 2008). The oldest known putative ceratosaur is Berberosaurus, which comes from the PliensbachianToarcian of Morocco (Allain et al., 2007). Allain et al. (2007) interpreted the fragmentary remains of Berberosaurus to represent the oldest abelisauroid, which would place it in a quite derived position within Ceratosauria. However, Carrano and Sampson (2008) instead argued that this taxon is the most basal ceratosaur, concordant with its stratigraphic position as the oldest unequivocal fossil of Ceratosauria. More recently, the phylogenetic analysis of $\mathrm{Xu}$ et al. (2009) found Berberosaurus as a more basal theropod, outside the Ceratosauria + Tetanurae clade. If correct, this would prompt a reinterpretation of neoceratosaur origins and early evolution.

The second of these major clades, Tetanurae, includes the largest carnivorous dinosaurs in most post-Early Jurassic ecosystems and later gave rise to birds (e.g., Sereno, 1999; Rauhut, 2003; Holtz et al., 2004; Smith et al., 2007). The oldest unequivocal tetanuran fossils are known from the early Middle Jurassic (Bajocian) of England (Waldman, 1974; Benson, 2008; Benson, 2010a). Carrano and Sampson (2004) suggested that a fragmentary knee joint from the late Sinemurian of England, included in the holotype of Scelidosaurus and figured by Owen (1861), may represent the oldest known tetanuran. However, there is no definitive anatomical evidence that this specimen is a tetanuran (Benson, 2010a). Nonetheless, because ceratosaurs and tetanurans are sister taxa, the presence of Berberosaurus, if a ceratosaur, implies that the tetanuran lineage also extends into the Early Jurassic.

Another possible clade of theropod dinosaurs also flourished during the Early Jurassic. The phylogenetic analysis of Smith et al. (2007) recovered a monophyletic "dilophosaurid" clade consisting of several medium-large-bodied Early Jurassic theropods, including Dilophosaurus (Fig. 3B), Cryolophosaurus, and Dracovenator. Each of these taxa possesses some form of distinctive cranial ornamentation, and features of these crests were important characters uniting the group in the phylogenetic analysis. However, Brusatte et al. (2010a) argued that Smith et al.'s (2007) character list too finely atomizes details of the cranial ornamentation, effectively over-representing the crest in the dataset and biasing the analysis towards finding a distinct clade of crested forms. As a result, when Brusatte et al. (2010a) reran the analysis using their own preferred system of scoring cranial crests the "dilophosaurid" clade disappeared. Additionally, this clade was not recovered by the comprehensive phylogenetic analysis of Nesbitt et al. (2009b), which samples a range of basal dinosaurs and theropods. Thus, the existence of a distinct "dilophosaurid" clade is currently a matter of debate among basal theropod workers. However, both groups of workers agree that none of these taxa (with the possible exception of Dilophosaurus) belongs to a coelophysoid clade, and therefore are theropods more closely related to ceratosaurs and tetanurans than to coelophysoids.

Alongside these more derived groups, coelophysoids remained common through the Early Jurassic before going extinct at or near the end of this time interval (Carrano et al., 2005; Ezcurra and Novas, 2007). Some of the most familiar coelophysoids, such as Coelophysis rhodesiensis, "Syntarsus" kayentakatae, and Segisaurus, are known from the Early Jurassic.

Finally, one puzzling specimen deserves comment. Zhao and Xu (1998) and Xu et al. (2001) described an incomplete lower jaw from the Early Jurassic Lufeng Formation of China as the oldest known therizinosauroid. Therizinosauroids are a bizarre clade of derived coelurosaurian theropods that, in the grand scheme of dinosaur evolution, are one of the closest relatives of birds (e.g., Sereno, 1999; Clark et al., 2004). If this jaw, which was described as a new genus (Eshanosaurus), does represent a therizinosauroid, then it would drag numerous derived theropod lineages into the Early Jurassic by virtue of ghost range extensions. None of these lineages is currently known from even fragmentary Early or Middle Jurassic fossils. However, the systematic affinities of Eshanosaurus have generated substantial controversy among dinosaur workers (e.g., Rauhut, 2003; Irmis, 2004; Barrett, 2009a). Most striking, Barrett (2009a) made a compelling argument that this specimen is poorly dated, and could be as young as Early Cretaceous in age. Therefore, the resolution of this enigma probably depends on the discovery of more complete, unambiguously associated, and well-dated material of Eshanosaurus, as well as additional discoveries of other Early Jurassic coelurosaur fossils.

\subsubsection{Early Jurassic theropods - paleobiology}

Relative to the Late Triassic, the Early Jurassic was a time of increased theropod diversity and morphological disparity. Several distinct theropod groups co-existed, and these differed in body size and general morphology. The remaining coelophysoids were mostly small, similar in body size to the familiar Late Triassic C. bauri. However, the Early Jurassic Dilophosaurus reached lengths of about $6 \mathrm{~m}$ and a mass of $400 \mathrm{~kg}$ (e.g., Welles, 1984; Peczkis, 1994). Cryolophosaurus was even larger, and is estimated at $6.5 \mathrm{~m}$ in length and $465 \mathrm{~kg}$ in mass (Smith et al., 2007). The fossil remains of Berberosaurus are fragmentary, but its femur is approximately $90 \%$ as large as that of Dilophosaurus (Allain et al., 2007).

Despite the large range in size and overall anatomy, all Early Jurassic theropods (with the possible exception of Eshanosaurus if indeed it is an Early Jurassic theropod) were likely carnivorous, judging from their shared arsenal of serrated teeth, sharp claws, and skeletons adapted for speed (e.g., long hindlimbs). The evolution of dietary diversity in theropods-which included piscivorous spinosauroids, omnivorous ornithomimosaurs, herbivorous therizinosauroids, and the bizarre oviraptorosaurs and alvarezsaurids-did not occur until later in the group's history. The preponderance of cranial ornamentation in Early Jurassic theropods suggests that visual display was important for these animals, but whether this is unusual compared to the normal range of archosaur cranial ornamentation is difficult to evaluate (Smith et al., 2007).

\subsubsection{Theropods across the Triassic/Jurassic boundary}

Theropods probably had a global distribution in the Late Triassic, because their remains are known from all regions with a good Late Triassic fossil record (southwestern USA, Germany, Poland, France, Argentina). Theropod distribution was clearly global in the Early Jurassic, with specimens known from North America, Europe, Asia, North Africa, and South Africa. Unfortunately, theropod remains are scarce enough that it is difficult to say much about latitudinal or other regional diversity patterns during the Late Triassic or Early Jurassic. 
A literal reading of the fossil record, as well as phylogenetic corrections that extend taxa back in time with ghost lineages, both indicate that there was a significant shift in theropod evolution across the Triassic-Jurassic boundary. Theropods were more taxonomically diverse in the Early Jurassic and evolved into a range of major clades and body plans during this time. How this diversification may relate to the Triassic-Jurassic extinction is unclear, but it is possible that theropods expanded in diversity, morphological disparity, and possibly ecological roles after the extinction of many carnivorous crurotarsan lineages (phytosaurs, ornithosuchids, rauisuchians) at or near the Triassic-Jurassic boundary (Olsen et al., 2002; Benton, 2004, 2005; Brusatte et al., 2008b). In any case, the overall picture of theropod rarity and morphological conservatism during the Late Triassic, and expansion in diversity and disparity in the Early Jurassic, argues against the hypothesis that theropods radiated rapidly soon after they originated (e.g., Hunt, 1991; Heckert and Lucas, 1995; Hunt et al., 1995; Carpenter, 1997).

\subsection{Sauropodomorphs}

\subsubsection{Late Triassic sauropodomorphs - fossil record and distribution}

Among Triassic dinosaurs, sauropodomorphs have one of the best fossil records in terms of taxonomic diversity and specimen abundance. Approximately twenty taxa are known from Late Triassic deposits on four continents (South America, Europe, Africa, Asia). These taxa fall into two general categories, which may or may not refer to discrete phylogenetic clades (see below). First, basal sauropodomorphs, commonly known as "prosauropods," were large, bipedal or quadrupedal herbivores or omnivores. Second, the more derived true sauropods were gigantic, fully quadrupedal, long-necked, barrel-chested herbivores.

The earliest sauropodomorphs are known from the late Carnian of South America. Martinez and Alcober (2009) recently described Panphagia protos based on a single well-preserved partial skeleton from the lower Ischigualasto Formation of northwestern Argentina. This taxon is currently the most basal sauropodomorph known, and lacks many of the derived characters present in other, more derived sauropodomorphs. An additional undescribed sauropodomorph is also present in the Ischigualasto Formation (Ezcurra, 2008). Until the discovery of Panphagia, Saturnalia tupiniquim from the upper Santa Maria Formation of southern Brazil (Langer et al., 1999, 2007c; Langer, 2003) was the most basal sauropodomorph known. This taxon is from strata that are biostratigraphically correlative with the Ischigualasto Formation (Langer, 2005; Langer et al., 2007b). Saturnalia is known from several specimens that together preserve most of the skeleton. This material provides our most complete look at the earliest sauropodomorphs and shows conclusively that Saturnalia shares many features with the rest of Sauropodomorpha.

By the end of the Norian, sauropodomorphs were both abundant and diverse in South America, Africa, and Europe. It is not uncommon for late Norian and Rhaetian formations from these continents to contain 3-6 penecontemporaneous sauropodomorph taxa (e.g., Los Colorados Formation: Galton and Upchurch, 2004). In contrast, no unquestionable sauropodomorph remains are known from North America until the Early Jurassic (Nesbitt et al., 2007).

The Triassic sauropodomorph species are phylogenetically diverse and include both basal forms as well as close relatives of the true sauropods. Unfortunately, the specific interrelationships of these taxa are still controversial, and two main competing phylogenetic hypotheses have been proposed. The first hypothesis suggests that most or all basal sauropodomorphs (i.e., non-sauropod sauropodomorphs) form a monophyletic group that is the sister taxon to Sauropoda (e.g., Sereno, 1999; Benton et al., 2000; Yates and Kitching, 2003; Galton and Upchurch, 2004; Sereno, 2007b; Upchurch et al., 2007a). In contrast, other studies find these taxa as a largely paraphyletic grade where some basal sauropodomorphs are closer to sauropods than they are to each other (e.g., Yates, 2003a,b, 2007a,b; Smith and Pol, 2007; Yates et al., 2010). Despite these disagreements, recent phylogenies agree in several aspects: that Panphagia, Saturnalia, Thecodontosaurus, Pantydraco, and Efraasia form successive branches at the base of Sauropodomorpha; that Coloradisaurus from the Late Triassic of Argentina forms a monophyletic clade with several Early Jurassic taxa including Massospondylus and Lufengosaurus; and the Late Triassic taxa Blikanasaurus, Lessemsaurus, Melanorosaurus, and Antetonitrus are more closely related to neosauropods ("true" sauropods) than to other basal sauropodomorphs (Smith and Pol, 2007; Upchurch et al., 2007a; Yates, 2007a,b; Yates et al., 2010).

\subsubsection{Late Triassic sauropodomorphs - paleobiology}

The earliest sauropodomorphs were small: Saturnalia has a femur length of $15 \mathrm{~cm}$ (Langer, 2003) and Panphagia was only slightly larger (Martinez and Alcober, 2009). Body size increased fairly early in sauropodomorph evolution. Efraasia and a majority of more derived sauropodomorphs have femur lengths above $50 \mathrm{~cm}$ (Carrano, 2006). The basal sauropods Antetonitrus and Lessemsaurus have femoral lengths of approximately $75 \mathrm{~cm}$ (Yates and Kitching, 2003; Pol and Powell, 2007a,b). Although Isanosaurus attavipachi from the Late Triassic of Thailand was about the same size, a 1.04-meter-long indeterminate sauropod humerus from the same strata demonstrates that sauropods reached truly gigantic sizes, equal to their Jurassic relatives, prior to the Triassic-Jurassic boundary (Buffetaut et al., 2002).

Associated with this increase in body size was a transformation from bipedal (the putative primitive dinosaurian condition) to quadrupedal locomotion. Although there is evidence that the earliest sauropodomorphs may have been facultatively quadrupedal (Langer et al., 2007b), most basal sauropodomorphs were unable to pronate their manus, which restricted their ability to walk quadrupedally (Bonnan and Senter, 2007). Nonetheless, more derived basal sauropodomorphs such as Aardonyx show specializations towards pronation (Yates et al., 2010) and the earliest sauropods were able to pronate their hands (e.g., Melanorosaurus and Antetonitrus), which along with a variety of other specializations indicates that these taxa were habitual if not obligate quadrupeds (Yates and Kitching, 2003; Bonnan and Yates, 2007; Yates et al., 2010). More derived Late Triassic sauropods like Isanosaurus were obligate quadrupeds, show a variety of graviportal specializations, and are very similar to other sauropods from the Early Jurassic (Buffetaut et al., 2000; Yates et al., 2010).

The final major functional change in early sauropodomorph evolution was the transformation from the primitive archosaurian state of carnivory to herbivory. Basal sauropodomorphs were traditionally interpreted as browsing herbivores based on their iguana-like teeth, long necks, and large body size (e.g., Galton, 1985). However, as pointed out by Barrett (2000), most of these features are ambiguous indicators of true herbivory. If comparisons with iguanid lizards are appropriate, it is likely that basal sauropodomorphs were omnivores (Barrett, 2000; Barrett and Upchurch, 2007). This view was strengthened by the discovery of the basal-most sauropodomorph, Panphagia, which shows few feeding specializations other than non-recurved teeth with large serrations/denticles (Martinez and Alcober, 2009). The teeth of Saturnalia show a similar condition (R.B.I., personal observation), and in fact are similar to those of the Triassic saurischian Eoraptor (Sereno et al., 1993). An increase in body size and the development of obligate quadrupedality through the evolution of basal sauropodomorphs is consistent with a trend towards a more herbivorous diet (Barrett and Upchurch, 2007). Specializations for obligate herbivory such as U-shaped jaws, spatulate tooth crowns with reduced denticles, and a lateral plate on the dentary only appear in the most basal sauropods (Barrett and Upchurch, 2007; Upchurch et al., 2007b). Although these features are only documented in Early Jurassic taxa (e.g., Upchurch et al., 2007b), they are present in taxa more basal than the Triassic Isanosaurus, 
indicating that obligate herbivory in sauropodomorphs must have evolved during the Late Triassic.

\subsubsection{Early Jurassic sauropodomorphs - fossil record and distribution}

Sauropodomorphs achieved a worldwide distribution during the Early Jurassic, and both basal sauropodomorphs ("prosauropods") and true sauropods thrived during this time. Anchisaurus is the earliest known sauropodomorph from North America (Yates, 2004), and Smith and Pol (2007) recently described Glacialisaurus from Antarctica. Glacialisaurus, Massospondylus from southern Africa, and Lufengosaurus from China are all part of a clade of basal sauropodomorphs that had its origins in the Late Triassic, with the Argentine Coloradisaurus (Smith and Pol, 2007; Yates, 2007a,b). Anchisaurus, along with Seitaad from western North America (Sertich and Loewen, 2010), Jingshanosaurus and Yunnanosaurus from China, and Aardonyx from South Africa appear to be typical "prosauropods", but may in fact be closely related to the true sauropods (Yates, 2004, 2007a,b; Yates et al., 2010). Basal sauropods also had a cosmopolitan distribution during the Early Jurassic, and include Chinshakiangosaurus and Gongxianosaurus from China, Vulcanodon from southern Africa, Tazoudasaurus from Morocco, and Barapasaurus from India. Although many of these lineages originated in the Late Triassic, the non-sauropod sauropodomorphs appear to have gone extinct at the end of the Early Jurassic. Indeed, no "prosauropods" are known from after this time period, and during the Middle Jurassic-Late Cretaceous large sauropods dominated the megaherbivore niche in most terrestrial ecosystems.

\subsubsection{Early Jurassic sauropodomorphs - paleobiology}

The discovery of Triassic sauropods demonstrated that most of the major changes in early sauropodomorph evolution, such as the development of quadrupedal locomotion and obligate herbivory, occurred prior to the Triassic-Jurassic boundary (see above). Thus, Early Jurassic sauropodomorphs represent further diversification of lineages that had already acquired these specializations earlier in their evolutionary history. In other words, the Early Jurassic was not a period of major new bodyplan evolution, but rather saw the modification of body types and lineages that had evolved much earlier.

During the Early Jurassic, sauropodomorphs continued to become more graviportal and increased in body size. Taxa such as Vulcanodon, Tazoudasaurus, and Barapasaurus had femoral lengths of well over a meter (Carrano, 2006; Allain and Aquesbi, 2008). The poor terrestrial fossil record during the latest Early and Middle Jurassic has limited our understanding of neosauropod origins and diversification, but it is likely that neosauropods originated in the late Early Jurassic. Perhaps the most significant paleobiological event in Early Jurassic sauropodomorph evolution is the disappearance of "prosauropod" type basal sauropodomorphs by the end of the epoch.

\subsubsection{Sauropodomorphs across the Triassic/Jurassic boundary}

Sauropodomorphs had a nearly cosmopolitan distribution by the end of the Norian (South America, Europe, Greenland, South Africa, southeast Asia, but not North America), and were present on all continents by the end of the Early Jurassic. Sauropodomorph remains are usually easily identifiable given that they are the largest terrestrial vertebrates during the Late Triassic-Early Jurassic. All recent phylogenetic hypotheses outlined above indicate that much of the diversification of basal Sauropodomorpha occurred in the Norian. Both typical "prosauropods" and early sauropods were present in the Late Triassic, and most of these lineages continued into the Early Jurassic. Thus, the Triassic-Jurassic extinction seemed to have little effect on sauropodomorph diversification, distribution, and abundance, even though the poor global terrestrial rock record of the latest Triassic (Rhaetian) limits conclusions about sauropodomorph evolution during this time.

\subsection{Ornithischians}

\subsubsection{Late Triassic ornithischians - fossil record and distribution}

Our understanding of Triassic ornithischians has undergone a radical revision in recent years. Prior to 2005, the Triassic ornithischian record was believed to include a number of taxa, including eight monospecific genera erected on the basis of isolated teeth from North America alone (Table 2). However, Parker et al. (2005) described the first non-dental material referable to one of these taxa, Revueltosaurus callenderi. These cranial and postcranial specimens lacked dinosaur features and were conclusively shown to belong to an herbivorous crurotarsan (crocodile-line) archosaur. Thus, any dental similarities between Revueltosaurus and ornithischians were independently acquired, and the preponderance of ornithischian-like teeth common in the Late Triassic of North America could no longer be definitely ascribed to ornithischians. This possibility had already been raised by the description of the basal dinosauromorph Silesaurus from the Carnian of Poland, which possessed low leaf-like teeth reminiscent of those of ornithischians (Dzik, 2003).

In combination, these two discoveries prompted a comprehensive reassessment of the Late Triassic ornithischian record (Irmis et al., 2007b; see also Butler et al., 2006a), which is summarized in Table 2. Following this reassessment, only three Late Triassic body fossil specimens are currently considered ornithischian, and all are from a relatively small geographical area in southern Gondwana.

First, Pisanosaurus is known from a partial skeleton that includes limited cranial material (Casamiquela, 1967; Bonaparte, 1976). Sereno (1991b) suggested that the holotype was a chimera of at least two taxa, but there seems to be little basis for this proposal (Irmis et al., 2007b). The phylogenetic position of Pisanosaurus is highly controversial: it has been identified as the most basal known ornithischian (Sereno, 1991b, 1999; Butler, 2005; Irmis et al., 2007a; Butler et al., 2008a), or as a possible heterodontosaurid (Bonaparte, 1976), and even its ornithischian affinities have been questioned (Thulborn, 2006; Irmis et al., 2007b). This uncertainty results from character conflict in the holotype: cranial material shares derived character states with ornithischians and specifically heterodontosaurids (e.g., the degree and pattern of occlusal wear facets) whereas the postcranial skeleton contains numerous plesiomorphic character states, including possibly an anteriorly directed pubis (seen in no other ornithischian: e.g., Sereno, 1986, 1999; Butler et al., 2008a; but see Irmis et al., 2007b).

Second, Báez and Marsicano (2001) described a tooth-bearing fragment of maxilla from Patagonia as a heterodontosaurid closely related to Heterodontosaurus from the Early Jurassic of South Africa (Fig. 3D). Although this fragment is poorly preserved, its heterodontosaurid identity has been tentatively accepted (Irmis et al., 2007b). Finally, Butler et al. (2007) and Butler (2010) described Eocursor from the lower Elliot Formation of South Africa, based upon a relatively complete skeleton of a single individual. Eocursor is the most completely known Triassic ornithischian and was identified as the sister taxon to Genasauria, a clade comprising most post-Triassic ornithischian diversity (Butler et al., 2007; Butler, 2010).

Tridactyl footprints from the Late Triassic of the USA, Europe and Africa have been identified as having been made by ornithischians (e.g., Biron and Dutuit, 1981; Mietto, 1985; Olsen and Baird, 1986; Dal Sasso, 2003; Knoll, 2004; Milàn and Gierlinski, 2004; Weishampel et al., 2004). However, a tridactyl pedal morphology similar to that of early ornithischians was present in many Triassic taxa, including basal saurischians, theropods, the earliest sauropodomorphs (e.g., Saturnalia: Langer, 2003), and dinosauromorphs (e.g., Silesaurus: Dzik, 2003). Thus, it is not possible to confirm the ornithischian identity of any of the reported footprints (Irmis et al., 2007b).

\subsubsection{Late Triassic ornithischians - ghost lineages and diversity}

Triassic ornithischian fossils are scarce, limiting our understanding of the early diversity of the clade. However, ghost lineages derived 


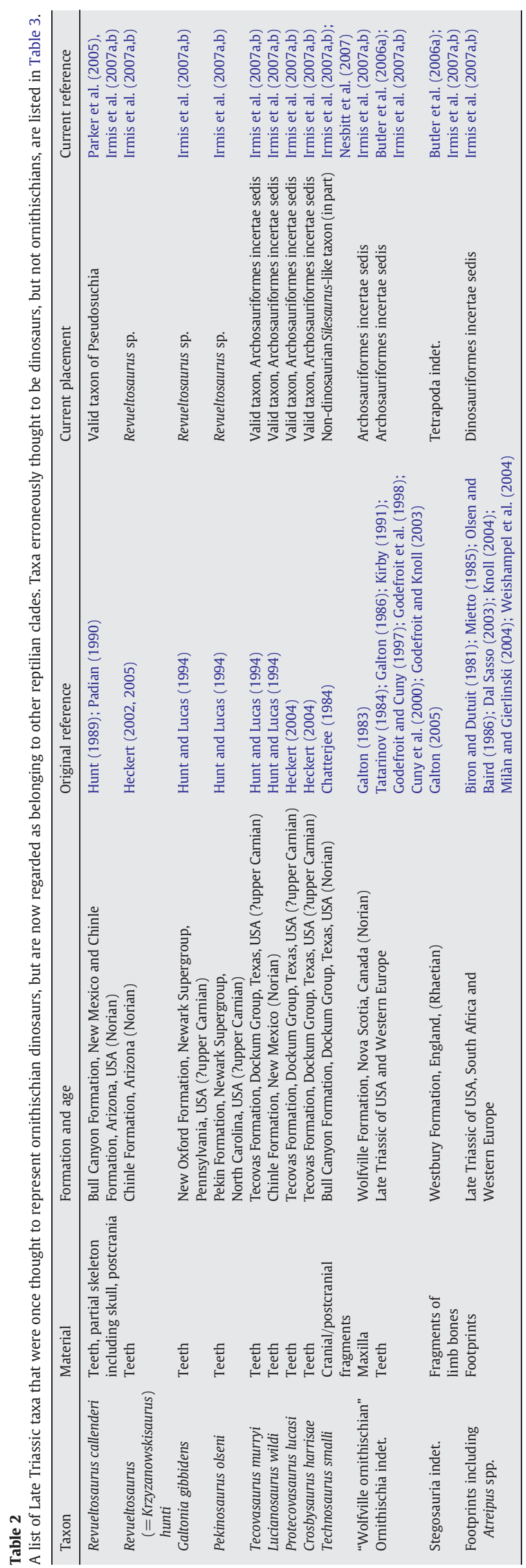

from phylogenies may indicate the presence of additional lineages for which fossil evidence has not yet been identified. The number of additional lineages that can be inferred depends upon the phylogeny chosen, as well as on interpretations of the phylogenetic position of fragmentary Late Triassic specimens.

Assuming that the maxilla described by Báez and Marsicano (2001) can be accurately referred to Heterodontosauridae (and/or Pisanosaurus ultimately proves to be a heterodontosaurid), the phylogeny of Sereno $(1986,1999)$ suggests that the major ornithischian clades Genasauria, Thyreophora, Neornithischia, Ornithopoda, Euornithopoda and Marginocephalia were also present prior to the Triassic/Jurassic boundary, implying a major Triassic ornithischian phylogenetic diversification despite apparent low numerical abundance (based on a dearth of fossils). Similarly, early origins of major ornithischian clades and high Triassic diversities are predicted by phylogenies that position heterodontosaurids as the sister taxon to Marginocephalia (e.g., Xu et al., 2006). However, if the Laguna Colorado maxilla does not represent a heterodontosaurid ornithischian, then the phylogenies of Sereno $(1986,1999)$ and Xu et al. (2006) would instead suggest that this diversification may have occurred in the earliest Jurassic.

An alternative view of ornithischian phylogeny differs primarily by positioning heterodontosaurids as non-genasaurian basal ornithischians (Butler, 2005; Butler et al., 2007, 2008a, 2010). This phylogeny implies an Early Jurassic origination date for Genasauria. In general, this phylogeny predicts later appearances for major ornithischian clades than do previous phylogenetic hypotheses, and a lower diversity of ornithischian clades present in the Late Triassic. As a result, this phylogeny fits the observed stratigraphic record more closely than do previous phylogenies (Wills et al., 2008).

\subsubsection{Late Triassic ornithischians - paleobiology}

The earliest ornithischians, such as Pisanosaurus and Eocursor, were small-bodied, with known specimens reaching just over a meter in body length (Bonaparte, 1976; Butler et al., 2007; Butler, 2010). Distal elements of the hindlimb (tibia, metatarsals) are elongate, suggesting well-developed cursorial abilities. Tooth-to-tooth occlusion and a buccal emargination were both present in Pisanosaurus (Sereno, 1991b). This latter character, which refers to the inset placement of the maxillary and dentary teeth, suggests the presence of a fleshy 'cheek', which has been viewed as a key ornithischian innovation (Galton, 1973; Sereno, 1997), and dental wear indicates a rapid acquisition of sophisticated jaw mechanics. By contrast, the cranial morphology of Eocursor is similar to that of Lesothosaurus (Sereno, 1991b) or Scutellosaurus (Colbert, 1981), with a low coronoid process of the lower jaw, a weakly inset dentary tooth row, a jaw joint which is only slightly offset below the level of the tooth row, and low, triangular teeth which lack systematic wear facets and possess enlarged denticles on mesial and distal surfaces. Barrett (2000) suggested that early ornithischians such as Lesothosaurus and heterodontosaurids may have been facultatively omnivorous, rather than strictly herbivorous, and this interpretation is also plausible for Pisanosaurus and Eocursor.

\subsubsection{Early Jurassic ornithischians - fossil record and distribution}

In stark contrast to the Late Triassic, Early Jurassic ornithischians are taxonomically and phylogenetically diverse, and are known from locally abundant and often excellently preserved material. The most diverse and important Early Jurassic ornithischian fauna is known from the upper Elliot Formation and overlying Clarens Formation of South Africa and Lesotho. This fauna includes the basal ornithischians Lesothosaurus (Thulborn, 1970a, 1971, 1972; Galton, 1978; Sereno, 1991b; Butler, 2005) and Stormbergia (Butler, 2005), and five named monospecific genera of heterodontosaurids (Heterodontosaurus, Abrictosaurus, Lycorhinus, Lanasaurus, Geranosaurus: Broom, 1911; Haughton, 1924; Crompton and Charig, 1962; Thulborn, 1970b, 1974; 
Gow, 1975; Hopson, 1975; Santa Luca et al., 1976; Santa Luca, 1980; Gow, 1990; Butler et al., 2008b). Recent reviews consider only three of the heterodontosaurid genera to be valid (Weishampel and Witmer, 1990; Norman et al., 2004c). However, the taxonomy of the Southern African heterodontosaurids is problematic: additional taxa may be present (RJB pers. obs.) and further work is needed. Although Early Jurassic ornithischian material is often described as rare, specimens representing over 60 southern African individuals (many undescribed) are known (RJB pers. obs.).

Within North America, substantial material of the early thyreophoran Scutellosaurus is present in the Kayenta Formation (SinemurianPliensbachian) of Arizona (Colbert, 1981; Rosenbaum and Padian, 2000), and is the most commonly recovered dinosaur from this formation (Tykoski, 2005). Large postcranial osteoderms indicate the presence of a second, larger, thyreophoran, and were referred to the genus Scelidosaurus by Padian (1989; see also Tykoski, 2005). An undescribed heterodontosaurid specimen (Attridge et al., 1985) is also known. The only other reported ornithischians from the Early Jurassic of North America are undescribed teeth from the McCoy Brook Formation of Nova Scotia (Shubin et al., 1994).

Thyreophoran ornithischians are well represented in the Early Jurassic of Europe. Scelidosaurus is known from multiple articulated and often nearly complete specimens from the Lower Lias (late Sinemurian) of England (Owen, 1861, 1863; Barrett, 2001), and Emausaurus is known from a single specimen from the Toarcian of Germany (Haubold, 1990).

Asian Early Jurassic ornithischians are known primarily from the Dark Red Beds of the Lower Lufeng Formation (Sinemurian) of China. Recent revisions of material from this formation have recognized only three specimens as ornithischian: the fragmentary holotypes of Tatisaurus (Simmons, 1965; Norman et al., 2007) and Bienosaurus (Dong, 2001), and an indeterminate fragmentary hindlimb (Irmis and Knoll, 2008). Tatisaurus and Bienosaurus are tentatively considered to represent basal thyreophorans (Norman et al., 2007). In contrast with the upper Elliot and Kayenta formations, ornithischians are exceptionally scarce components of the Lower Lufeng assemblage.

Early Jurassic terrestrial faunas from South America are poorly known. However, Barrett et al. (2008) described teeth and a distal tibia referable to Ornithischia from either the Early or Middle Jurassic of Venezuela.

\subsubsection{Early Jurassic ornithischians - paleobiology}

Heterodontosaurids were more abundant and diverse in the Early Jurassic than at any other time in their evolutionary history, and a range of cranial morphologies were present (e.g., Hopson, 1975; Weishampel and Witmer, 1990). This suggests that a variety of cranial mechanisms and feeding styles may have been important in enabling a number of heterodontosaurid genera to coexist (e.g. in the upper Elliot Formation of southern Africa). Among other Early Jurassic ornithischians, an orthal mechanism with some interlocking of the upper and lower dentitions has been postulated for Lesothosaurus (Thulborn, 1971), whereas Barrett (2001) suggested a 'puncture-crushing' mechanism for Scelidosaurus. It is possible that most Early Jurassic ornithischians were omnivorous (Barrett, 2000), rather than strictly herbivorous.

Most Early Jurassic ornithischians (e.g. heterodontosaurids, Lesothosaurus, Scutellosaurus) were apparently small-bodied, with the largest known individuals reaching around 1-1.5 $\mathrm{m}$ in length. However, the Early Jurassic marks the appearance of the first moderately large ornithischians. Adults of Scelidosaurus were at least $4 \mathrm{~m}$ in length, and Stormbergia probably reached lengths of around $3 \mathrm{~m}$. Ornithischians of similar size were probably also present in the Kayenta Formation of Arizona (Padian, 1989; RJB pers. obs.)

Most Early Jurassic ornithischians probably utilized both quadrupedal and bipedal gaits, as argued for heterodontosaurids (Santa Luca, 1980; Weishampel and Witmer, 1990), Lesothosaurus (Norman et al. 2004a), and Scutellosaurus (Colbert, 1981; Norman et al., 2004b), and as suggested by the probable ornithischian ichnogenus Anomoepus (Olsen and Rainforth, 2003). Scelidosaurus is generally regarded as an obligate quadruped (Norman et al., 2004b), suggesting that a reversal to this condition had occurred in the thyreophoran lineage by the Sinemurian. However, Gierlinski (1999) has suggested, based upon ichnological evidence, that large basal thyreophorans such as Scelidosaurus may have been capable of at least occasional bipedal locomotion.

\subsubsection{Ornithischians across the Triassic/Jurassic boundary}

During the Early Jurassic, ornithischians achieved a global distribution, with definite body fossils known from Africa, Europe, North America, and Asia. Although poorly dated, body fossils may indicate the existence of ornithischians in South America in the Early Jurassic. Ornithischians are both relatively abundant and diverse within the upper Elliot Formation of southern Africa and the Kayenta Formation of the USA (see above). In contrast, ornithischian fossils remain highly scarce relative to saurischians in the Lower Lufeng Formation of China, suggesting that ornithischian abundance varied geographically (Irmis and Knoll, 2008). Early armored dinosaurs (thyreophorans) were diverse and are known from North America, Asia, and Europe, but are absent from the southern African record, suggesting some degree of provinciality in early ornithischian faunas.

There was undoubtedly a dramatic increase in ornithischian abundance across the Triassic-Jurassic boundary. By the Early Jurassic ornithischians are relatively diverse, abundant, and globally distributed. Major ornithischian clades such as Genasauria, Thyreophora and Neornithischia can be identified. The exact timing of this diversification is problematic, because of the poorly constrained dating of many Late Triassic and Early Jurassic sequences, but it does appear that ornithischians are scarce in, or absent from, most latest Triassic (Norian/Rhaetian) assemblages, but relatively abundant in earliest Jurassic (Hettangian/Sinemurian) assemblages. How this diversification might relate to proposed extinction events at the Triassic-Jurassic boundary remains uncertain. However, a number of herbivorous clades went extinct at this time, which may have vacated ecological niches into which ornithischians were able to radiate (Olsen et al., 2002; Butler et al., 2007; Brusatte et al., 2008b).

\subsection{Taxa often mistaken as dinosaurs}

Throughout the Late Triassic dinosaurs evolved alongside their close relatives, the crurotarsan (crocodile-line) archosaurs (Fig. 7). These two groups were heavily convergent on each other, in some cases eerily so (Nesbitt and Norell, 2006), and as a result many fragmentary specimens of crurotarsans have been mistaken for dinosaurs, and vice versa (Tables 2 and 3). Many such specimens, especially isolated teeth, were formally assigned to various dinosaurs in the pre-cladistic era of archosaur systematics. However, Benton (1986b) demonstrated that many of these so-called dinosaur specimens from the Triassic actually represent crurotarsans (Fig. 7). Additionally, Benton (1986b) showed that putative Early and Middle Triassic dinosaur footprints, identified across Europe, could not be unambiguously identified as dinosaur tracks.

"Dinosaur-like" crurotarsans can still be problematic. For example, Chatterjee (1993) announced the discovery of Shuvosaurus from the Late Triassic of Texas, which he interpreted as the oldest member of the ornithomimid lineage, a group of theropods mostly restricted to the Cretaceous. As ornithomimids are deeply nested within Theropoda, this discovery suggested that many lineages of carnivorous dinosaurs were present, but unknown from fossils, in the Late Triassic. However, Nesbitt and Norell (2006) and Nesbitt (2007) demonstrated that Shuvosaurus and its close relative Effigia are actually members of the crurotarsan lineage.

Other studies have mistaken even more basal reptiles as among the 'oldest dinosaurs.' For example, Flynn et al. (1999) reported two new sauropodomorph dinosaurs from the early Late Triassic of Madagascar. 
A

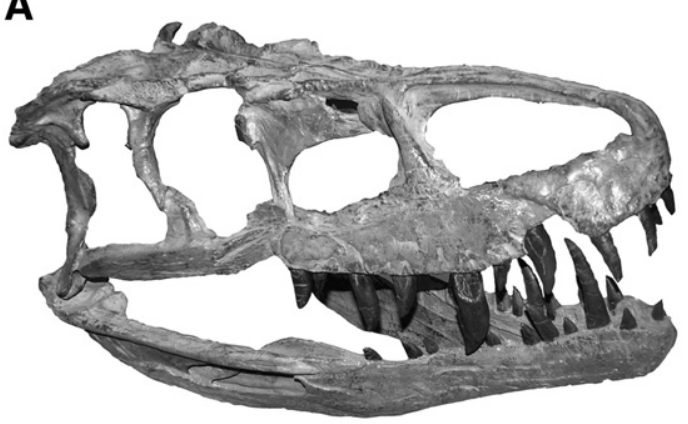

B

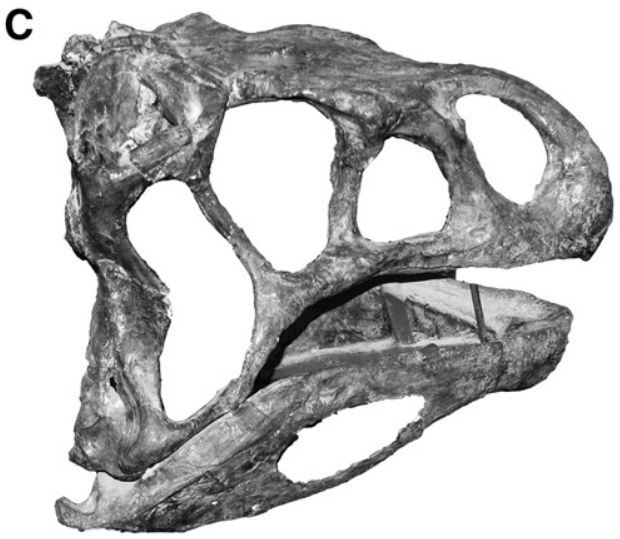

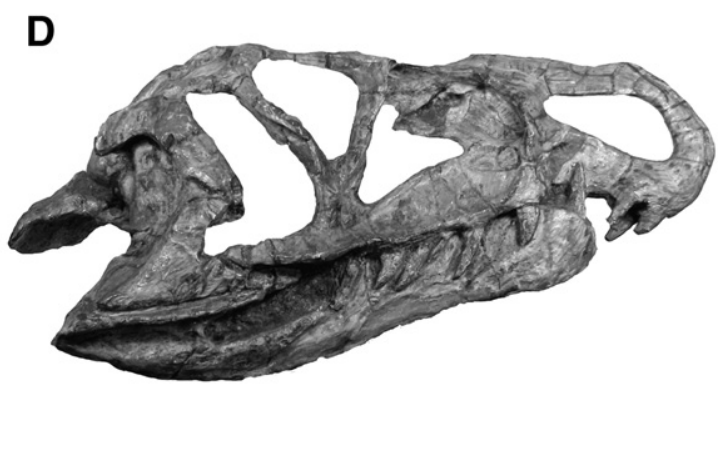

Fig. 7. A montage of crurotarsan (crocodile-line) archosaurs convergent on the earliest dinosaurs. A, Batrachotomus, a large, quadrupedal, rauisuchian predator from the Ladinian of Germany; B, Postosuchus, a large, quadrupedal, rauisuchian predator from the Norian of the United States; C, Lotosaurus, a mid-sized, quadrupedal, sail-backed omnivore from the Anisian of China; D, Riojasuchus, a mid-sized, quadrupedal or bipedal, swift predator from the Norian of Argentina. Figure delineated by Stephen Brusatte.

Additional material and a careful reevaluation of the specimens demonstrate that the purported 'sauropodomorph' material belongs to a taxon only distantly related to dinosaurs that shares uncanny modifications of the skull with plant-eating, large-bodied dinosaurs (Flynn et al., 2008; Flynn et al., 2010). Similarly, Nesbitt et al. (2007) demonstrated that several supposed dinosaurs from the Late Triassic of North America actually represent dinosauromorphs, the closest relatives to dinosaurs rather than bona fide members of the group.

This historical review, although brief, testifies to both a practical problem in identifying Late Triassic specimens and a remarkable fact about evolution during this period. Although most large-bodied terrestrial reptiles of the Jurassic and Cretaceous were dinosaurs, a number of different Triassic groups converged on the same general

Table 3

A list of Late Triassic taxa that were once thought to represent dinosaurs, but are now regarded as belonging to other reptilian clades. Taxa erroneously thought to be ornithischian dinosaurs are listed separately in Table 2. Technosaurus is listed in both tables, as its holotype is a chimaera (Nesbitt et al., 2007).

\begin{tabular}{lll}
\hline Taxon & Current placement & Reference \\
\hline Azendohsaurus & Basal archosauromorph & Flynn et al. (2008) \\
Eucoelophysis & $\begin{array}{l}\text { Silesaurus-like } \\
\text { dinosauromorph }\end{array}$ & $\begin{array}{l}\text { Nesbitt et al., 2007; Ezcurra } \\
\text { (2007) }\end{array}$ \\
Ornithosuchus & Crurotarsan archosaur & Gauthier (1986); Sereno (1991a) \\
Postosuchus & Crurotarsan archosaur & Long and Murry (1995) \\
Protoavis & Numerous taxa & Nesbitt et al. (2007) \\
Saltopus & Dinosauromorph & Rauhut and Hungerbühler (2000) \\
Shuvosaurus & Crurotarsan archosaur & Nesbitt and Norell (2006) \\
Spinosuchus & Basal archosauriform & Nesbitt et al. (2007) \\
Spondylosoma & Crurotarsan archosaur? & Galton (2000) \\
Technosaurus & Silesaurus-like & Nesbitt et al. (2007) \\
& dinosauromorph & \\
Teratosaurus & Crurotarsan archosaur & Benton (1986b) \\
\hline
\end{tabular}

body plans, including animals closely related to modern crocodylians (Fig. 7). Although this often makes it difficult to identify fragmentary specimens, it suggests that the Late Triassic was a unique time in terrestrial vertebrate evolution during which different groups iteratively evolved the same generalized morphologies (Nesbitt and Norell, 2006; Nesbitt, 2007).

\section{The dinosaur radiation: a historical review}

Until the 1980s, most authors (e.g., Colbert, 1964; Romer, 1966; Bakker, 1972; Charig, 1972, 1984) pictured the radiation of the dinosaurs as part of an evolutionary relay of successive faunal replacements throughout the Triassic. This was the favored viewpoint for three main reasons:

(1) As noted earlier, most authors considered that the dinosaurs were a polyphyletic assemblage and hence that dinosaurs arose several times, essentially convergently, as a result of similar competitive pressures.

(2) The origin of the dinosaurs was seen as a drawn-out affair that started early in the Middle Triassic and involved extensive and long-term competition. The dinosaur ancestors were regarded as superior animals, with advanced locomotory adaptations (erect gait: Charig, 1972, 1984) or physiological advances (e.g., warm-bloodedness: Bakker, 1972) that progressively competed with, and caused the extinction of, all of the synapsids and basal archosaurs, that lacked such superior features.

(3) The first appearance of dinosaurs was seen as a great advance that must have been the mark of some kind of competitive process. It had commonly been assumed that the evolution of life was in some way 'progressive,' and that more recent plants and animals are inevitably better than those that went before. We discuss this further, below. 
Beginning in the 1980s, many scientists began to take a different view. Benton (1983, 1986a, 1994) argued that the dinosaurs radiated after ecospace had been cleared during the end-Carnian extinction event (which now is likely dated within the Norian, because of the redating of strata discussed above), and that the dinosaurs did not establish their pre-eminence after a long period of competition with precursor groups. This view was supported by several lines of evidence:

(1) The fossil record does not show a gradual takeover, but apparently two expansions after extinction events, the first at the end of the Carnian (or early Norian) when the dominant herbivores, the rhynchosaurs and dicynodonts, became dramatically depleted, and were replaced in the Norian by basal sauropodomorphs, and then at the end of the Triassic, when most crurotarsans died out, and large theropods and armored dinosaurs radiated in the Early Jurassic.

(2) The first dinosaurs had all or most of the supposed "key characters" (upright stance, etc.) that were thought to help them outcompete other groups, but they did not take over at once (Sereno, 1999). During the Carnian, all three major dinosaurian lineages were present, but they did not radiate until much later.

(3) The "superior adaptations" of dinosaurs were probably not so profound as was once thought. For instance, many other archosaurs also evolved erect gait in or by the Late Triassic, and yet they died out (e.g. aetosaurs, rauisuchians, ornithosuchids, and some early crocodylomorphs).

(4) There were other extinctions at the end of the Carnian or within the early Norian. The Dicroidium flora of the southern hemisphere gave way to a worldwide conifer flora about this time (see above). There were turnovers in marine communities, particularly in reefs, and there was a shift from pluvial (heavy rainfall) climates to arid climates throughout much of the world (Simms and Ruffell, 1990b). The climatic and floral changes may have caused the extinctions of the dominant herbivorous tetrapods.

(5) The idea that simple competition can drive the replacement of one major group by another is an oversimplification. Competition between higher taxa ("families" or "orders") of animals is very different from the ecological observation of competition within or between species in an ecosystem. In paleontological examples such as this, competition has often been assumed to have been the mechanism, but the evidence has generally been shown to be weak (Benton, 1987).

Ideas of competition and superiority stemmed from the deepseated views of many distinguished architects of the Modern Synthesis (e.g., Theodosius Dozhansky, George Gaylord Simpson, Julian Huxley) that evolution was progressive (Gascoigne, 1991). Dobzhansky et al. (1977, p. 508) defined progress in evolution as "systematic change in a feature belonging to all members of a sequence in such a way that posterior members of the sequence exhibit an improvement of that feature." Such views emerged naturally from Darwin's world view that evolution was competitive, and that a new species could arise only by supplanting a pre-existing species. Darwin, in his unpublished Natural Selection manuscript (see Stauffer, 1975, p. 208), compared the present-day diversity of species to a number of apples floating on the surface of a barrel filled with water. The surface of the water is packed with floating apples, and it is impossible to add a new apple without displacing one that is already there. Such ideas were at the base of many branches of ecological theory, including the classic Lotka-Volterra models of the 1930s, the theory of island biogeography (MacArthur and Wilson, 1967), and the logistic models of global marine biodiversity (Sepkoski, 1996).

The tension between selection and contingency, or, as Darwin put it, between selection and environment, goes on today. The Red Queen model of evolution (Van Valen, 1973) sees most of macroevolution (long-term evolution of large clades) driven by biotic interactions, although the physical environment is allowed a place. An opposing view, termed the Court Jester model (Barnosky, 2001), is that changes in climate and topography, and unpredictable events (contingency), contribute much more to the larger patterns, and especially to wholesale extinctions and many major diversifications following such crises. The question is how much of the tree of life, of modern biodiversity, and large-scale geographic patterns of distribution are mediated by physical environmental factors, and how much by competition and predation in ecosystems (Benton, 2009)?

In exploring classic examples of diversifications and biotic replacements (Gould and Calloway, 1980; Benton, 1987; Roy, 1996), most turned out to be best explained as responses to contingent events such as mass extinctions. Competition was rarely invoked as a simplistic clade vs. clade process, but rather at a more refined level of, for example, differential response to a crisis. Whatever the final outcome of these debates about the most influential drivers of largescale evolution, a key lesson has been not to make unsupported assumptions, and to focus on quantifiable data (taxonomic diversity, faunal abundance, morphological disparity), and to do so within a sound chronologic, stratigraphic, and phylogenetic framework. Because dinosaurs are a major group that has been well studied and for which an abundance of phylogenetic, stratigraphic, and morphological data exist, they are an ideal test case for examining macroevolutionary patterns over time.

\section{The macroevolutionary pattern of the dinosaur radiation}

\subsection{Introduction}

There is a rich historical legacy of debate regarding the early evolutionary history of dinosaurs. The Triassic fossil record and the toolkit of analytical methods available to paleontologists have changed greatly over the course of this debate. Many of the first scientists to offer hypotheses on the dinosaur radiation based their ideas on a literal reading of the fossil record combined with intuition based on experience and assumptions about how macroevolution works over long time scales. Over the past decade scientists have aimed to understand biases in the fossil record, worked to incorporate a phylogenetic framework into their studies, and begun to utilize a wide array of analytical techniques to quantify macroevolutionary patterns.

Many of these methods have been used to examine the radiation of dinosaurs. Perhaps the most important result of these studies is an understanding that the dinosaur radiation is more complex than often assumed (e.g., Brusatte et al., 2008b). Evolutionary radiations are not single events that can be described with broad platitudes, but have many different components that are often decoupled from each other. For instance, a clade may originate long before it speciates into a number of lineages, becomes numerically abundant in its ecosystem(s), or evolves into a wide range of different body types or ecological roles. These various components-lineage origination (cladogenesis), faunal abundance, taxonomic diversity, and morphological disparityare distinctive measures of biodiversity that may or may not be related to each other. Each has been used to describe the radiation of dinosaurs in some form or another, but they must be considered side-by-side for an integrative picture of the early history of dinosaurs.

\subsection{Lineage origination, cladogenesis, and phylogeny}

The oldest unequivocal dinosaur fossils are known from the Carnian (see above) and are approximately 230 million years old. However, as with any observed fossil occurrences, this is only a minimum estimate for the origination of the dinosaur lineage. In reality, it is likely that dinosaurs extended further back in time, and the duration of this missing record can be estimated by ghost lineages on the phylogenetic tree of dinosaurs and their closest relatives (e.g., Norell, 1992). Because dinosaurs and their sister taxon had to originate at the same point in time (by definition), the discovery of 
a sister taxon fossil older than the oldest known dinosaur will in effect extend the range of dinosaurs (or more accurately, their stem lineage) earlier in time (Norell, 1992, 1993).

Most recent phylogenetic analyses recover a sister group relationship of Dinosauria and a clade of mostly herbivorous dinosauromorphs centered on Silesaurus, Sacisaurus, and Eucoelophysis (e.g., Irmis et al., 2007a; Brusatte et al., 2008a; Nesbitt et al., 2009b, 2010; Brusatte et al., 2010b). Most members of Silesauridae are Carnian-Norian in age (e.g., Sullivan and Lucas, 1999; Dzik, 2003; Ezcurra, 2007; Ferigolo and Langer, 2007). However, Nesbitt et al. (2010) recently reported the discovery of a new member of the silesaurid clade, Asilisaurus, in the ?late Anisian of Tanzania. Thus, the presence of Asilisaurus in the Anisian implies that the lineage leading to Dinosauria originated by this time (Sidor et al., 2008; Nesbitt et al., 2010).

Within Dinosauria, the oldest ornithischian (Pisanosaurus: Bonaparte, 1976; Irmis et al., 2007b) and sauropodomorph (Saturnalia: Langer et al., 1999) are from the Carnian. The oldest unequivocal theropods, including Coelophysis, Zupaysaurus, and Liliensternus, are from the Norian. Coelophysis has long been considered to extend into the Carnian (e.g., Tykoski and Rowe, 2004), but revised radioisotopic dates for the Chinle Formation of the southwestern United States indicate that all localities where Coelophysis fossils are found are Norian in age at the oldest (Irmis and Mundil, 2008). However, as theropods are the sister taxon to sauropodomorphs, their ghost lineage extends into the Carnian. Indeed, if the controversial basal dinosaurs Herrerasaurus and Eoraptor do represent true theropods (see above), then Carnian specimens are already known (Rogers et al., 1993). Ghost lineages are unable to extend the ornithischian and sauropodomorph lineages back further from the Carnian, but it would not be surprising if unequivocal ornithischians, sauropodomorphs, theropods, or stem saurischians, do eventually come to light in Middle Triassic assemblages.

A phylogenetic perspective also gives insight into the pace of the dinosaur radiation. Recent discoveries of non-dinosaurian dinosauromorphs in Norian assemblages have greatly increased the stratigraphic range of the closest dinosaurian cousins (Irmis et al, 2007a). Previously these animals were thought to have gone extinct at or around the time that dinosaurs themselves originated (e.g., Sereno and Arcucci, 1994a,b). The new discoveries show that dinosaurs and their closest cousins persisted side-by-side for up to 20 million years, indicating that the rise of dinosaurs-the process by which dinosaurs became the preeminent terrestrial vertebrates at the expense of closely related groups-was a prolonged affair.

In summary, dinosaurs are first known from the Carnian but their stem lineage extends at least into the Anisian (Middle Triassic) based on ghost lineages. The major subgroups of dinosaurs are first known from the Carnian and early Norian, and none of these lineages can yet be confidently extended earlier than the Carnian. Thus, the current picture is one of early dinosaur origination (possibly in the Middle Triassic) followed by a delayed splitting of major dinosaur subgroups sometime during the Carnian. The dinosaur radiation itself was gradual, and proceeded in many steps, not sudden.

\subsection{Taxonomic diversity and significant diversification shifts}

In macroevolutionary studies "diversity" refers strictly to the number of taxa (usually species, genera, or higher taxa such as families), usually within a certain time bin or a certain area. This is different from lineage origination: a group can be present but contain very few species, and thus exhibits low diversity. Measuring diversity is normally quite straightforward, as it necessitates nothing more than counting taxa over time or space, and in some cases correcting for missing lineages unknown in the fossil record but implied by phylogeny (ghost lineages: Norell, 1992).

On the other hand, "diversification" is a broad, and often vague, umbrella term that is used in many different ways. Oftentimes researchers will refer to "significant diversification events" in a group's evolutionary history. These are moments in time when a group speciates (or avoids extinction) at a pace or in a pattern that differs from the more normal "background" tempo of evolution. Although seemingly vague, these events can be identified by statistical tests that compare an observed phylogeny or diversity profile with a null expectation for how groups should split and speciate over time if splitting is random. This null expectation is usually based on a "birthdeath" model that assumes each lineage has an equal, but independent, probability of splitting at any given time over the course of a group's evolution (see Chan and Moore, 2002; Nee, 2006; Ricklefs, 2007; Purvis, 2008 for more details). In essence, a certain time interval or a certain part of a cladogram can be identified as exhibiting "significant diversification" if it differs from the null model.

The taxonomic diversity of dinosaurs over time has long been a subject of interest, especially for those scientists studying the duration and magnitude of the extinction of non-avian dinosaurs at the end of the Cretaceous (e.g., Dodson, 1990). Diversity measurements continually change as new fossils are discovered and specimens are reinterpreted (for instance, compare the dinosaur diversity measurements of Dodson (1990) and Wang and Dodson (2006)). A profile of dinosaur diversity over time was recently provided by Lloyd et al. (2008), who were also the first authors to provide a phylogenetic correction to diversity measures across all Dinosauria (based on a supertree of dinosaur phylogeny, which as a "summary tree" is a broad and inexact proxy for a correction) and examine the potential sampling biases implicit in the dinosaur fossil record. Their diversity curves, based both on observed fossils ("taxic estimate") and observed counts corrected for ghost lineages ("phylogenetic estimate"), indicate a steady increase in diversity from the Carnian through the Early Jurassic (Table 4). Their statistical subsampling technique, which attempts to standardize sampling in order to remove biases that result from temporal variation in the quality of the fossil record, suggests that diversity was steady across the Carnian and Norian but jumped in the Early Jurassic (Lloyd et al., 2008: fig. 2b). (See also the recent phylogenetically-corrected diversity analysis presented by Barrett et al. (2009)).

Lloyd et al. (2008) also used their dinosaur supertree to ask two important questions: (1) which specific nodes (branching events) represent significant diversification shifts?; and (2) are significant diversification shifts concentrated in any specific interval of time? By comparing their cladogram to one expected under the null "birthdeath" model, Lloyd et al. (2008) identified several nodes that exhibit significant diversification shifts. These are essentially nodes that are significantly more speciose than their sister taxon, which is a violation of the null model that assumes random splitting over time (see Chan and Moore, 2002, 2005; Jones et al., 2005). Importantly, these significant nodes are concentrated in the first third of dinosaur history, and most of them in the Late Triassic and Early Jurassic (Lloyd et al., 2008: fig. 3a,c), a result corroborated by statistical tests. Thus, the Late Triassic and Early Jurassic was a critical interval for dinosaur diversification, especially compared to the remainder of the history of dinosaurs.

Table 4

Dinosaur diversity by time (data from Lloyd et al., 2008; Brusatte et al., 2008b).

\begin{tabular}{lcccl}
\hline & Carnian & Norian & Early Jurassic $^{1}$ & Early Jurassic $^{2}$ \\
\hline Taxic & 9 & 22 & 26 & 27 \\
Phylogenetic & 14 & 9 & 8 & 13 \\
Total & 23 & 31 & 34 & 40 \\
\hline
\end{tabular}

"Taxic" indicates observed fossil occurrences, with dates taken from Weishampel et al. (2004). "Phylogenetic" indicates the observed data plus a correction for ghost lineages (phylogenetic history of a taxon unpreserved in the fossil record but implied by the supertree of Lloyd et al., 2008). "Total" is a summation of taxic and phylogenetic measures. Early Jurassic estimates are calculated without (1) and with (2) inclusion of Eshanosaurus, a controversial derived theropod that, if correctly identified, drags several lineages into the Early Jurassic. 


\subsection{Morphological disparity and morphospace occupation}

Morphological disparity refers to the range of morphologies and body types exhibited by a group of organisms. Disparity measures something quite different from lineage origination and diversity: a group could be present and/or taxonomically diverse, but may only exhibit a narrow array of body types and anatomical variability. Alternatively, a group could have very few species (low diversity), each characterized by a highly unique morphology (high disparity).

Disparity can be measured in several ways, using either morphometric data or discrete characters, such as those used in phylogenetic analyses (Wills et al., 1994). The goal in each case is to represent the overall morphology of a set of organisms. These morphological measurements or characters are then subjected to multivariate statistical analysis, which ordinates taxa in a multidimensional space (a "morphospace": Raup, 1965; McGhee, 1999; Erwin, 2007). In essence, a morphospace is akin to a morphological "map," which graphically represents how similar and different taxa are from each other in their body plans. Statistical tests can then be used to determine if certain groups of organisms (usually binned either taxonomically or by time) have a greater diversity of morphologies than other groups. In statistical terms, morphological diversity can be quantified in many ways, but the two most common methods calculate range and variance statistics for the different bins (Wills et al., 1994; Ciampaglio et al., 2001). Range measures denote the entire spread of morphological variation (the size of morphospace occupied by the group), whereas variance measures indicate average dissimilarity among members of the group (the spread of the group in morphospace).

The morphological disparity of Late Triassic and Early Jurassic dinosaurs, as well as other contemporaneous archosaur groups, was measured by Brusatte et al. (2008a,b) (Figs. 8 and 9). These studies indicate that dinosaur disparity increased over time, from the Carnian through the Early Jurassic (Fig. 9). The main jump in disparity was between the Carnian and Norian, which is deemed significant by statistical tests, whereas there was only a slight and non-significant

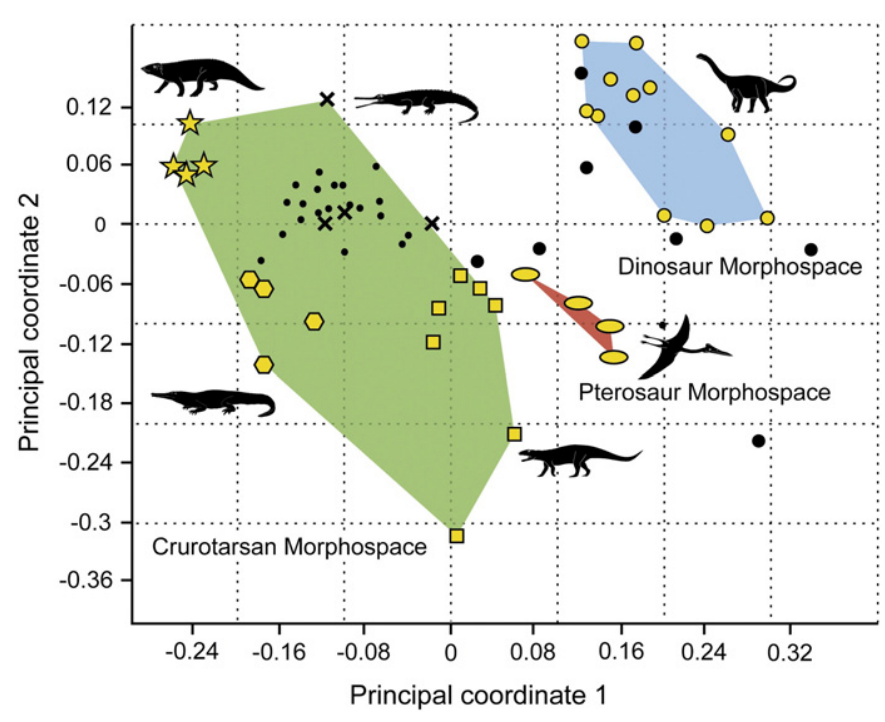

Fig. 8. A morphospace for Triassic archosaurs, based on Brusatte et al. (2008a). Three general clusters of taxa are denoted: crurotarsans (crocodile-line archosaurs), pterosaurs, and dinosaurs. Crurotarsan morphospace is significantly larger than dinosaur morphospace, as well as avemetatarsalian (dinosaur + pterosaur + dinosauromorph morphospace), meaning that crurotarsans were occupying a larger range of body plans and morphologies than dinosaurs during the Late Triassic. Large outlined circles, dinosaurs; ovals, pterosaurs; squares, poposauroid rauisuchians; hexagons, phytosaurs; stars, aetosaurs; crosses, crocodylomorphs; small solid circles, "rauisuchid" rauisuchians; large solid circles, non-dinosaurian dinosauromorphs and Scleromochlus. Plot delineated by Stephen Brusatte and Simon Powell (University of Bristol). increase from the Norian to the Early Jurassic despite the extinction of many supposed dinosaur competitors at the Triassic-Jurassic boundary.

Brusatte et al. (2008a,b) also calculated the disparity of the crurotarsan archosaurs, which were exceptionally abundant and diverse in the Late Triassic, lived alongside early dinosaurs for tens of millions of years, and in many cases were eerily morphologically convergent with dinosaurs (Fig. 7). These facts suggest that crurotarsans and dinosaurs were "competitors" during the Late Triassic, in the sense that they were similar animals that lived alongside each other and probably competed for similar resources (e.g., Nesbitt and Norell, 2006; Nesbitt, 2007; Brusatte et al., 2008a,b). Importantly, crurotarsans were significantly more disparate than dinosaurs throughout the Late Triassic, and it was only after the Triassic-Jurassic extinction that dinosaur disparity overtook crurotarsan disparity (Figs. 8 and 9). In other words, crurotarsans were exploring a wider range of body plans, morphologies, and diets than Triassic dinosaurs. These results hold if strict sister taxa-in this case Avemetatarsalia and Crurotarsi, the two main lines of archosaur phylogeny-are compared. Brusatte et al. (2008a) used this result to argue that early dinosaur history was more a matter of contingency than prolonged, gradual outcompetition of competitor groups.
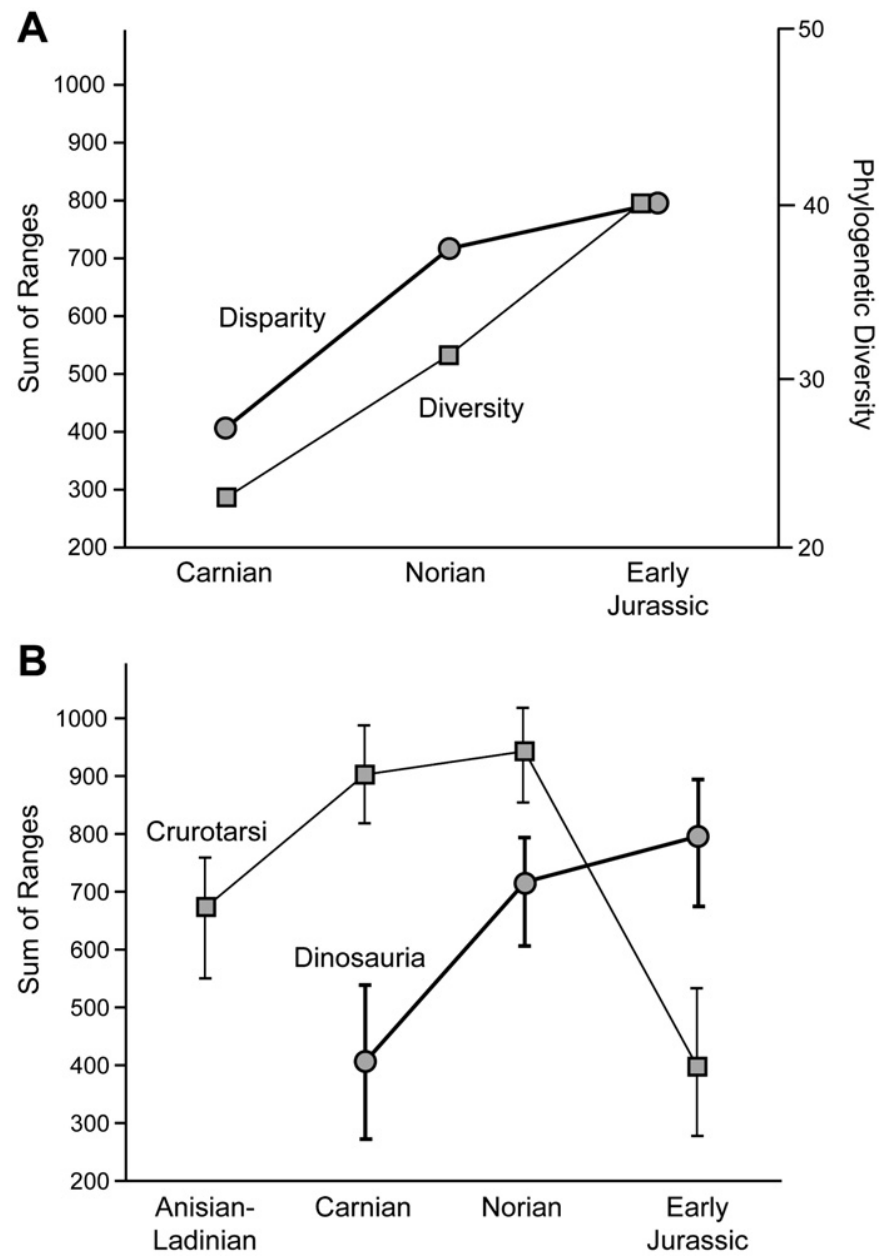

Fig. 9. A plot of archosaur taxonomic diversity and morphological disparity over time, based on Brusatte et al. (2008b). A, diversity and disparity for dinosaurs across the Late Triassic and Early Jurassic; B, disparity for dinosaurs and crurotarsans across the Late Triassic and Early Jurassic. Morphological disparity (Norian) peaked earlier than taxonomic diversity (Early Jurassic) in the evolutionary radiation of dinosaurs. Crurotarsans had a significantly higher disparity (occupied more morphospace) than dinosaurs across the Late Triassic, but after the Triassic-Jurassic extinction dinosaurs occupied significantly more morphospace. Plots delineated by Stephen Brusatte and Simon Powell (University of Bristol). 
However, it must be noted that the decrease in crurotarsan disparity in the Early Jurassic may partially be an artifact of sampling, because Brusatte et al. (2008b) did not include several morphologically disparate crocodylomorphs from the Kayenta Formation: the Edentosuchus-like form (Sues et al., 1994: fig. 16.3) and the goniopholid Calsoyasuchus (Tykoski et al., 2002).

One aspect of Brusatte et al.'s (2008a,b) study deserves further comment. Dinosaurs and crurotarsans were shown not to overlap in morphospace (Fig. 8), which could be conceived as evidence against direct competition. However, this conclusion is premature for three reasons. First, the figured morphospace plots only depict the first two (of many) multivariate axes, and dinosaurs and crurotarsans do overlap on most of these axes. Second, disparity analyses have a phylogenetic bias, and the separation of dinosaurs and crurotarsans on the first (most important) multivariate axis is likely the result of phylogeny alone, just as the separation of taxon clusters in some morphometric analyses is due to size alone. Third, as outlined by Stayton (2006), the mere overlap or non-overlap of taxa in morphospace is inadequate for assessing convergence.

\subsection{Faunal abundance}

Absolute numerical abundance is a straightforward measure: the percentage of individuals or biomass belonging to a certain species or group in an ecosystem. Once again, this need not be tied to lineage origination, diversity, or disparity. For instance, it is easy to imagine a situation in which a certain group is exceptionally abundant in an ecosystem, but all of this abundance is due to a single species (low diversity) with a single body plan (low disparity).

Unfortunately, measuring absolute faunal abundance in fossil assemblages is exceptionally difficult. First, preserved fossil assemblages are rarely unbiased records of extinct ecosystems, but rather represent a collection of specimens that has passed through numerous taphonomic and preservational filters. Second, the time and resources needed to undertake a complete census of a fossil assemblage can be astronomical, especially if specimens are spread across different museums and have been collected by different groups. Understandably, abundance studies (at least of fossil vertebrates) are rare and play only a small role in current Mesozoic vertebrate paleobiological research programs.

Only one study has attempted to measure the absolute abundance of early dinosaurs and other Triassic and Jurassic vertebrates from many sites across the globe. Benton (1983) relied on detailed databases of museum collections and correspondence from local experts to chart the abundance of early dinosaurs and their contemporaries. The most important results from this study are: (1) that dinosaurs were rare in the Carnian but their abundance spiked in the Norian; (2) crurotarsan archosaurs were more numerically abundant than dinosaurs in many Late Triassic ecosystems; (3) the relative abundance of dinosaurs and crurotarsans varied by latitude during the Late Triassic; and (4) by the Early Jurassic dinosaurs were the most abundant large terrestrial vertebrates in ecosystems across the globe. Although Benton's (1983) database is by now severely outdated and geographically restricted, we see no reason to doubt these general patterns he observed for the regions studied given our own experience with fieldwork and collections work worldwide. However, there are clear deviations from this trend, particularly in North America, where dinosaur abundance remains low throughout the Norian (Irmis et al., 2007a).

\subsection{Rates of morphological change}

Evolutionary biologists utilize many different measures of evolutionary rate, which aim to quantify the "speed" of evolution. The most common approach is to measure the rates of origination and extinction of lineages over time, which gives an overall rate of diversification (e.g., Ricklefs, 2007; Fröbisch, 2008; Ruta and Benton, 2008). Other rate metrics quantify the rate of molecular sequence change (e.g., Mindell and Thacker, 1996; Lavin et al., 2005) or various aspects of continuous phenotypic change, such as body size (e.g., Garland, 1992; Gingerich, 1993; Pagel, 1998; Roopnarine, 2003; O'Meara et al., 2006).

An additional rate metric, which is seldom used but potentially illuminating, is the rate of discrete morphological character change over time. Measuring this rate necessitates the optimization of discrete morphological characters onto a cladogram, which allows for the calculation of a rate of change (characters changing/time) for each branch on the tree (Wagner, 1997). This procedure is still in its infancy, and awaits a formalized statistical protocol for hypothesis testing (Oakley, 2003), but has been used to examine the early evolutionary history of tetrapods (Ruta et al., 2006) and dinosaurs (Brusatte et al., 2008a).

Brusatte et al. (2008a) used a framework phylogeny of Triassic archosaurs and a database of over 400 anatomical characters to measure the average rate of change for different taxonomic groups and time intervals. They found that dinosaurs and their crurotarsan "competitors" had statistically indistinguishable rates of change, a sign that neither group was outpacing the other during the Late Triassic. Additionally, they found that the rate of change within dinosaurs was significantly higher in the Carnian than in the Norian, consistent with a long-hypothesized prediction that evolutionary rates are highest during the earliest part of a clade's history (Valentine, 1980; Schluter, 2000; Gould, 2002).

\section{The evolutionary radiation of dinosaurs: current status}

The five macroevolutionary measures described above-lineage origination, taxonomic diversity, morphological disparity, faunal abundance, and evolutionary rates-give an integrated and synthetic picture of the evolutionary radiation of dinosaurs. Quantitative studies of the dinosaur radiation, which subject observed fossils, morphological features, and phylogeny to rigorous statistical protocols, are still in their infancy. However, the message that has emerged from recent studies is clear and consistent: the radiation of dinosaurs was a prolonged affair, not a sudden takeover, and was much more complex than often assumed.

Most importantly, the radiation of dinosaurs (or any group for that matter) must be viewed as a patchwork quilt comprised of many components. Traditionally paleontologists have treated the rise of dinosaurs-either explicitly or implicitly-as a single event (e.g., Bakker, 1971, 1972; Bakker and Galton, 1974; Charig, 1984), which is often explained by generalized mechanisms such as "competition," "opportunism," or "superiority." In other words, the prevailing debate has long been whether the rise of dinosaurs resulted from some sort of innate superiority, which enabled dinosaurs to outcompete other reptiles, or was a matter of contingent good luck on a grand scale (see review in Benton, 2004). As is often the case, such debates inevitably result in simplification and polarization: complex concepts are distilled down to buzzwords and researchers retreat to their own corners instead of generating new data.

The new quantitative toolkit unmistakably exposes the dinosaur radiation as a complex process that unfolded over tens of millions of years. Dinosaurs originated long before they became taxonomically diverse, morphologically disparate, or numerically abundant in their ecosystems (Fig. 9). Morphological disparity and high rates of character change spiked early in dinosaur history, long before and out-of-step with taxonomic diversity. This decoupling of disparity and diversity, and the burst of disparity before diversity, is typical for many evolutionary radiations (Erwin, 2007). Likely competitors to early dinosaurs explored a larger range of body types throughout the Late Triassic and evolved at indistinguishable rates, and even the closest cousins of dinosaurs persisted alongside their better-known 
relatives for up to 20 million years. Very clearly, different components of the dinosaur radiation proceeded at different paces and were decoupled from each other, as has also been seen in many other groups (see review in Erwin, 2007). With this pattern now unmasked, it is foolish to try to reduce the first 50 million years of dinosaur history into a single platitude.

With that being said, and keeping in mind the intricacies of the debate, most of us still favor a more-or-less "opportunistic" view of the evolutionary radiation of dinosaurs. This view itself is much more complex than its one-word slogan may imply, and unfortunately is often condensed by the press (and in some cases by our own misguided words) into an argument for random "good luck" and nothing more. The argument boils down to a dichotomy between contingency and continuity, a view of evolution that has been well articulated by Gould (1989), Alvarez (1997), and others. Both contingency and continuity play a role in the history of life, but the fundamental question in the current paper is this: which process was more important in the early history of dinosaurs? Was the rise of dinosaurs a gradual process by which things changed incrementally over time? In other words, were dinosaurs destined to become dominant and preeminent vertebrates, by virtue of certain characteristics or abilities, once they originated? Or, did dinosaurs take advantage of one or several contingencies of earth history, which could have never been predicted when the first dinosaur arose sometime in the Middle Triassic?

Our view of the dinosaur radiation is in line with Jablonski's (1986, 1991, 2001, 2005, 2008) view of macroevolution: that "successful" organisms must navigate both background and mass extinction events in order to survive and prosper. The dinosaurs were clearly successful during the first $30+$ million years of their history in the Middle and Late Triassic. But so were the crurotarsan archosaurs and the close dinosauromorph cousins to dinosaurs. In fact, based on their larger morphospaces, greater range of body types and diets, and numerical dominance in many ecosystems, it could be argued that the crurotarsans were doing better than the dinosaurs during the Late Triassic. It was only after the Triassic-Jurassic mass extinction that dinosaurs could truly stake a claim as the preeminent terrestrial vertebrates, as it was during the Early Jurassic when they achieved numerical dominance in terrestrial ecosystems across the globe and enjoyed their most significant period of diversification. The same cannot be said of the crurotarsans, which were hit hard by the mass extinction and truncated to only a single lineage, the crocodylomorphs (although this lineage re-radiated later in the Jurassic and Cretaceous into a range of body forms that have yet to be investigated by disparity analysis).

Thus, there was nothing "inevitable" or "predestined" about their later success when dinosaurs first arose: they had to traverse both a prolonged journey throughout the Late Triassic and a sudden period of global devastation at the Triassic-Jurassic extinction. Although debatable, dinosaurs also appear to have weathered a more minor extinction at the Carnian-Norian boundary or in the early Norian. Dinosaurs only had "good luck" in the sense that they were able to endure the extinction(s) whereas some of their contemporary competitors were not. We do not deny that dinosaurs may have survived because one or several "important" characters-indeed, it is likely that the marked asymmetry in dinosaur and crurotarsan survival into the Early Jurassic was due to differences in growth, metabolism, or locomotion. The important distinction is that these characters were not "key innovations" that allowed, or drove, dinosaurs to either rapidly blossom when they first originated or gradually outcompete other groups over long time scales. They were simply features that came in handy when an unpredictable global meltdown struck.

In closing, we distill our view of the dinosaur radiation into the following statement: there was nothing predestined or superior about dinosaurs when they first arose, and without the contingency of various earth-history events during the early Mesozoic, the Age of Dinosaurs might have never happened.

\section{The evolutionary radiation of dinosaurs: future directions}

The origin and early evolution of dinosaurs have long been subjects of fascination and continue to be a central focus of research in the paleontology community. Current work on early dinosaur history is proceeding at a rapid pace, with particular emphasis on both primary data collection and synthetic studies that aim to place early dinosaurs in a phylogenetic, paleoenvironmental, and macroevolutionary context.

Over the past 25 years, perceptions of dinosaur origination and early history have changed with the discovery of new specimens, both new taxa and fossils of previously described species. There is no reason to think that this trend will change, especially as Triassic and Jurassic rocks are being explored at a remarkable rate, new localities are consistently being discovered, and localities known for over a hundred years are still producing new and exciting specimens. In particular, field exploration in Tanzania (Sidor et al., 2008; Nesbitt et al., 2010), Madagascar (Flynn et al., 2008), Poland (Dzik and Sulej, 2007; Dzik et al., 2008), the southwestern United States (Irmis et al., 2007a; Nesbitt et al., 2009a,b), Argentina (Martinez and Alcober, 2009), and Brazil (Ferigolo and Langer, 2007) has already yielded important new material, and should continue to do so throughout the near future. Additional fieldwork is underway across western and central Europe, South America, and Asia, and these regions-or other as yet unknown areas-may prove to be the next frontier of early dinosaur discovery.

Similarly, perceptions of early dinosaur evolution have changed in concert with changing views on the phylogeny of dinosaurs and their closest relatives. Several large-scale analyses of archosaur and dinosaur phylogeny are underway (e.g., Brusatte et al., 2008c; Nesbitt et al. 2009b, 2010; Brusatte et al., 2010b; SJN, unpublished data; RBI, unpublished data), and paleontologists are just beginning to experiment with large phylogenetic datasets. These analyses will be instrumental in reconstructing the pattern of anatomical character change on the line to dinosaurs (e.g., Nesbitt et al., 2009a,b), as well as the biogeographic distribution of dinosaurs during the Triassic and Jurassic (e.g., Upchurch et al., 2002; Butler et al., 2006b; Nesbitt et al., 2009b).

A robust understanding of early dinosaur evolution also relies on geological data. It is essential to not only understand the climatic and environmental backdrop of early dinosaur history, but also the absolute and relative ages of dinosaur fossils and entire dinosaur assemblages. Such temporal information is critical to large-scale macroevolutionary studies, such as those that examine the diversity and evolutionary rates of dinosaurs over time. The Triassic and Early Jurassic are notoriously lacking in precise radioisotopic dates, and correlations between formations on both local and global scales is extremely difficult (e.g., Furin et al., 2006; Mundil, 2007). Previously, scientists have relied on organisms themselves to date and correlate the rocks, but many of these correlations have proved to be unreliable (Rayfield et al., 2005; Martz and Small, 2006; Irmis and Mundil, 2008; Rayfield et al., 2009). Perhaps more so than new specimens, precise radioisotopic dates of units within key dinosaur-bearing Triassic and Jurassic formations, such as the Chinle and Elliot Formations, are of the utmost importance.

Finally, the fundamental goal for paleontologists working on early dinosaur history is to understand exactly how dinosaurs ascended from a marginal group of small Triassic reptiles to the dominant terrestrial vertebrates of the Jurassic and Cretaceous. Questions such as these are difficult to answer, and perhaps intractable, but if solved may go a long way in helping scientists understand large-scale evolutionary processes and the dynamics of evolutionary radiations. For workers interested in these big-picture questions, there is perhaps no more ideal group to focus on than dinosaurs. Broad-scale 
macroevolutionary analyses must be based on a firm foundation of anatomical, phylogenetic, and geological data. These types of study are in their infancy (e.g., Brusatte et al., 2008a,b), and so far have looked at the diversity, evolutionary rates, and morphospace occupation of dinosaurs over time. Large-scale studies of early dinosaur biogeography, lineage-level dinosaur and archosaur extinctions during the early Mesozoic, and regional faunal and ecological differences remain to be done. It is these types of study, in association with new fossil discoveries and renewed phylogenetic and geological analyses, which promise an exciting future for dinosaur research.

\section{Acknowledgements}

We thank F. Wallien for the invitation (to SLB) to contribute a review article on the origin of dinosaurs. We are indebted to Frank Ippolito and Simon Powell for assistance with figures (see figure captions) and thank Bill Parker and an anonymous reviewer for helpful comments. We thank our numerous collaborators on projects relating to early dinosaur history, in particular P. Barrett, R. Benson, M. Ezcurra, M. Langer, G. Lloyd, G. Niedźwiedzki, W. Parker, L. Porro, and M. Ruta. We are indebted to countless curators and collections managers at museums across the globe who have assisted with our research. For many of us, our interest in dinosaur origins and Triassic-Jurassic paleontology was stoked and fostered by our academic advisors: P. Sereno (SLB), K. Padian (SJN, RBI), P. Olsen (SJN, SLB), P. Barrett (RJB), D. Norman (RJB), P. Upchurch (RJB), as well as MAN and MJB. SLB is funded by a Marshall Scholarship for study in the UK (University of Bristol) and an NSF Graduate Research Fellowship (Columbia University), and specimen visits pertinent to this project were funded by the Paleontological Society, Jurassic Foundation, SYNTHESYS, and Bob Savage Memorial Fund (University of Bristol). RBI's research has been funded by a variety of sources, including an NSF Graduate Research Fellowship, University of California Museum of Paleontology Samuel \& Doris Welles Research Fund, University of California Berkeley Department of Integrative Biology, Jurassic Foundation, Palaeontological Association Sylvester-Bradley Award, American Museum of Natural History Theodore Roosevelt Memorial Fund, and Society of Vertebrate Paleontology Bryan Patterson Memorial Grant, and the University of Utah. RJB is funded by an Alexander von Humboldt Postdoctoral Fellowship. Aspects of this project were funded by NSF ATOL 0228693 to MAN. Writing this manuscript necessitated considerable division of labor. Although all of the authors were involved in the writing and editing of the final draft, particular sections were first written by specific authors: SLB (biological setting, theropods, macroevolution, current status, future directions); SJN (dinosaur diagnosis, ambiguous dinosaur taxa, sauropodomorphs, future directions); RBI (geological setting, ambiguous dinosaur taxa, sauropodomorphs); RJB (ornithischians); MJB (historical review, competition models); MAN (feathers).

\section{References}

Algeo, T.J., Ingall, E., 2007. Sedimentary $\mathrm{C}_{\text {org: }} \mathrm{P}$ ratios, paleocean ventilation, and Phanerozoic atmospheric $\mathrm{pO}_{2}$. Palaeogeography, Palaeoclimatology, Palaeoecology 256, 130-155.

Allain, R., Aquesbi, N., 2008. Anatomy and phylogenetic relationships of Tazoudasaurus naimi (Dinosauria, Sauropoda) from the late Early Jurassic of Morocco. Geodiversitas $30,345-424$

Allain, R., et al., 2004. A basal sauropod dinosaur from the Early Jurassic of Morocco. Comptes Rendus Palevol 3, 199-208.

Allain, R., Tykoski, R., Aquesbi, N., Jalil, N.-E., Monbaron, M., Russell, D., Taquet, P., 2007. An abelisauroid (Dinosauria: Theropoda) from the Early Jurassic of the High Atlas Mountains, Morocco, and the radiation of ceratosaurs. Journal of Vertebrate Paleontology 3, 610-624.

Alvarez, W., 1997. T. rex and the Crater of Doom. Princeton University Press, Princeton, NJ. Andrews, C.W., 1921. On some remains of a theropodous dinosaur from the Lower Lias of Barrow-on-Soar. Annals and Magazine of Natural History 8, 570-576.

Arcucci, A.B., Coria, R.A., 2003. A new Triassic carnivorous dinosaur from Argentina. Ameghiniana 40, 217-228.
Attridge, J., Crompton, A.W., Jenkins, F.A., 1985. The southern African Liassic prosauropod Massospondylus discovered in North America. Journal of Vertebrate Paleontology 5, 128-132.

Báez, A.M., Marsicano, C.A., 2001. A heterodontosaurid ornithischian dinosaur from the Upper Triassic of Patagonia. Ameghiniana 38, 271-279.

Bai, Z., Yang, J., Wang, G., 1990. Yimenosaurus, a new genus of Prosauropoda from Yimen County, Yunnan Province [in Chinese]. Yuxiwenbo (Yuxi Culture and Scholarship) $1,14-23$.

Bakhurina, N.N., Unwin, D.M., 1995. A preliminary report on the evidence for 'hair' in Sordes pilosus, an Upper Jurassic pterosaur from Middle Asia. In: Sun, A., Wang, Y, (Eds.), Sixth Symposium on Mesozoic Terrestrial Ecosystems and Biota. Short Papers. China Ocean Press, Beijing, pp. 79-82.

Bakker, R.T., 1971. Dinosaur physiology and the origin of mammals. Evolution 25, 636-658.

Bakker, R.T., 1972. Anatomical and ecological evidence of endothermy in dinosaurs. Nature 238, 81-85.

Bakker, R.T., Galton, P.M., 1974. Dinosaur monophyly and a new class of vertebrates. Nature 248, 168-172.

Barnosky, A.D., 2001. Distinguishing the effects of the Red Queen and Court Jester on Miocene mammal evolution in the northern Rocky Mountains. Journal of Vertebrate Paleontology 21, 172-185.

Barrett, P.M., 2000. Prosauropod dinosaurs and iguanas: speculations on the diets of extinct reptiles. In: Sues, H.-D. (Ed.), Evolution of Herbivory in Terrestrial Vertebrates. Cambridge Univ. Press, Cambridge, pp. 42-78.

Barrett, P.M., 2001. Tooth wear and possible jaw action of Scelidosaurus harrisonii Owen and a review of feeding mechanisms in other thyreophoran dinosaurs. In: Carpenter, K. (Ed.), The Armored Dinosaurs. Indiana University Press, Bloomington, IN, pp. 25-52.

Barrett, P.M., 2004. Sauropodomorph dinosaur diversity in the upper Elliot Formation (Massospondylus range zone: Lower Jurassic) of South Africa. South African Journal of Science 100, 501-503.

Barrett, P.M., 2009a. The affinities of the enigmatic dinosaur Eshanosaurus deguchiianus from the Early Jurassic of Yunnan Province, People's Republic of China. Palaeontology 52, 681-688.

Barrett, P.M., 2009b. A new basal sauropodomorph dinosaur from the upper Elliot Formation (Lower Jurassic) of South Africa. Journal of Vertebrate Paleontology 29, 1032-1045.

Barrett, P.M., Upchurch, P., 2007. The evolution of feeding mechanisms in early sauropodomorph dinosaurs. Special Papers in Palaeontology 77, 91-112.

Barrett, P.M., Upchurch, P., Wang, X.-L., 2005. Cranial osteology of Lufengosaurus huenei Young (Dinosauria: Prosauropoda) from the Lower Jurassic of Yunnan, People's Republic of China. Journal of Vertebrate Paleontology 25 (4), 806-822.

Barrett, P.M., Upchurch, P., Zhou, X.-D., Wang, X.-L., 2007. The skull of Yunnanosaurus huangi Young, 1942 (Dinosauria: Prosauropoda) from the Lower Lufeng Formation (Lower Jurassic) of Yunnan, China. Zoological Journal of the Linnean Society 150, 319-341.

Barrett, P.M., Butler, R.J., Novas, F.E., Moore-Fay, S., Moody, J.M., Clark, J.M., SanchezVillagra, M.R. 2008. Dinosaur remains from the La Quinta Formation (Lower or Middle Jurassic) of the Venezuelan Andes. Paläontologishe Zeitscrift 82, 163-177.

Barrett, P.M., McGowan, A.J., Page, V., 2009. Dinosaur diversity and the rock record. Proceedings of the Royal Society of London, Series B 276, 2667-2674.

Benson, R.B.J., 2008. A redescription of "Megalosaurus" hesperis (Dinosauria, Theropoda) from the Inferior Oolite (Bajocian, Middle Jurassic) of Dorset, United Kingdom. Zootaxa 1931, 57-67.

Benson, R.B.J., 2010a. The osteology of Magnosaurus nethercombensis (Dinosauria, Theropoda) from the Bajocian (Middle Jurassic) of the United Kingdom and a reexamination of the oldest records of tetanurans. Journal of Systematic Palaeontology 8 , 131-146.

Benson, R.B.J., 2010b. A description of Megalosaurus bucklandii (Dinosauria: Theropoda) from the Bathonian of the United Kingdom and the relationships of Middle Jurassic theropods. Zoological Journal of the Linnean Society 158, 882-935.

Benson, R.B.J., Barrett, P.M., Powell, H.P., Norman, D.B., 2008. The taxonomic status of Megalosaurus bucklandii (Dinosauria, Theropoda) from the Middle Jurassic of Oxfordshire, UK. Palaeontology 51, 419-424.

Benton, M.J., 1983. Dinosaur success in the Triassic: a noncompetitive ecological model. The Quarterly Review of Biology 58, 29-55.

Benton, M.J., 1984. The relationships and early evolution of the Diapsida. Symposium of the Zoological Society of London 52, 575-596.

Benton, M.J., 1985. Classification and phylogeny of the diapsid reptiles. Zoological Journal of the Linnean Society 84, 97-164.

Benton, M.J., 1986a. The Late Triassic tetrapod extinction events. In: Padian, K. (Ed.), The Beginning of the Age of Dinosaurs; Faunal Change Across the Triassic-Jurassic Boundary. Cambridge Univ. Press, Cambridge, pp. 303-320.

Benton, M.J., 1986b. The Late Triassic reptile Teratosaurus, a rauisuchian, not a dinosaur. Palaeontology 29, 293-301.

Benton, M.J., 1987. Progress and competition in macroevolution. Biological Review 62, 305-338.

Benton, M.J., 1994. Late Triassic to Middle Jurassic extinctions among continental tetrapods: testing the pattern. In: Fraser, N.C., Sues, H.-D. (Eds.), In the Shadow of the Dinosaurs. Cambridge University Press, Cambridge, pp. 366-397.

Benton, M.J., 1995. Diversification and extinction in the history of life. Science 268, $52-58$.

Benton, M.J., 1999. Scleromochlus taylori and the origin of dinosaurs and pterosaurs. Philosophical Transactions of the Royal Society of London, Series B 354, 1423-1446.

Benton, M.J., 2003. When Life Nearly Died: the Greatest Mass Extinction of All Time. Thames and Hudson, London. 336 pp. 
Benton, M.J., 2004. Origin and relationships of Dinosauria, In: Weishampel, D.B. Dodson, P., Osmólska, H. (Eds.), The Dinosauria, 2nd edition. University of California Press, Berkeley, CA, pp. 7-24.

Benton, M.J., 2005. Vertebrate Palaeontology, 3rd edition. Blackwell, Oxford. 455 pp.

Benton, M.J., 2009. The Red Queen and the Court Jester: species diversity and the role of biotic and abiotic factors through time. Science 323, 728-732.

Benton, M.J., Clark, J.M., 1988. Archosaur phylogeny and the relationships of the Crocodylia. In: Benton, M.J. (Ed.), The Phylogeny and Classification of the Tetrapods: Systematics Association Special, Volume 35A, pp. 295-338.

Benton, M.J. Juul, L. Storrs, G.W. Galton, P.M., 2000. Anatomy and systematics of the prosauropod dinosaur Thecodontosaurus antiquus from the Upper Triassic of southwest England. Journal of Vertebrate Palaeontology 20, 77-108.

Benton, M.J., Tverdokhlebov, V.P., Surkov, M.V., 2004. Ecosystem remodelling among vertebrates at the Permain Triassic boundary in Russia. Nature 432, 97-100.

Bergman, N.M., Lenton, T.M., Watson, A.J., 2004. COPSE: a new model of biogeochemical cycling over Phanerozoic time. American Journal of Science 304, 397-437.

Berner, R.A., 2006. GEOCARBSULF: a combined model for Phanerozoic atmospheric $\mathrm{O}_{2}$ and $\mathrm{CO}_{2}$. Geochimica et Cosmochimica Acta 70, 5653-5664.

Biron, P.E., Dutuit, J.M., 1981. Figurations sédimentaires et traces d'activité au sol dans le Trias de la formation d'Argana et de l'Ourika (Maroc). Bulletin du Muséum National d'Histoire Naturelle: Sect. C: Sciences de la Terre, Paléontologie, Géologie, Mineralogie, vol. 4, pp. 399-427.

Bittencourt, J.de.S., Kellner, A.W.A., 2009. The anatomy and phylogenetic position of the Triassic dinosaur Staurikosaurus pricei Colbert, 1970. Zootaxa 2079, 1-56.

Bonaparte, J.F., 1969. Does nuevas "faunas" de reptiles Triasicos de Argentina, International Gondwana Symposium 1967. UNESCO, Mar del Plata.

Bonaparte, J.F., 1975. Neuvos materials de Lagosuchus talampayensis Romer (Thecodontia-Pseudosuchia) y su significado en el origin de los Saurischia. Acta Geológica Lilloana 13, 5-90.

Bonaparte, J.F., 1976. Pisanosaurus mertii Casamiquela and the origin of the Ornithischia. Journal of Paleontology 50, 808-820.

Bonaparte, J.F., 1978. Coloradia brevis n. g. et n. sp. (Saurischia-Prosauropoda), dinosaurio Plateosauridae de la Formación Los Colorados, Triásico Superior de La Rioja, Argentina. Ameghiniana 15, 327-332.

Bonaparte, J.F., 1997. El Triasico de San Juan - La Rioja Argentina y sus dinosaurios. Museo Argentino de Ciencias Naturales, Buenos Aires. 190 pp.

Bonaparte, J.F., 1999. Evolución de las vértebras presacras en Sauropodomorpha. Ameghiniana 36, 115-187.

Bonaparte, J.F., Vince, M., 1979. El hallazgo del primer nido de dinosaurios Triasicos, (Saurischia, Prosauropoda), Triasico Superior de Patagonia, Argentina. Ameghiniana $16,173-182$.

Bonaparte, J.F., Ferigolo, J., Ribeiro, A.M., 1999. A new early Late Triassic saurischian dinosaur from Rio Grande do Sul State, Brazil. Proceedings of the Second Gondwanan Dinosaur Symposium: National Science Museum Monographs, Tokyo, vol. 15, pp. 89-109.

Bonaparte, J.F., Brea, G., Schultz, C.L., Martinelli, A.G., 2007. A new specimen of Guaibasaurus candelariensis (basal Saurischia) from the Late Triassic Caturrit Formation of southern Brazil. Historical Biology 19, 73-82.

Bonnan, M.F., Senter, P., 2007. Were the basal sauropodomorph dinosaurs Plateosaurus and Massospondylus habitual quadrupeds? Special Papers in Palaeontology 77, 139-155.

Bonnan, M.F., Yates, A.M., 2007. A new description of the forelimb of the basa sauropodomorph Melanorosaurus: implications for the evolution of pronation, manus shape and quadrupedalism in sauropod dinosaurs. Special Papers in Palaeontology 77, 157-168.

Brack, P., Rieber, H., Nicora, A., Mundil, R., 2005. The Global Boundary Stratotype Section and Point (GSSP) of the Ladinian Stage (Middle Triassic) at Bagolino (southern Alps, northern Italy) and its implications for the Triassic time scale. Episodes 28 233-244.

Brady, L.F., 1935. Preliminary note on the occurrence of a primitive theropod in the Navajo. American Journal of Science 30, 210-215.

Brady, L.F. 1936. A note concerning the fragmentary remains of a small theropod recovered from the Navajo Sandstone in northern Arizona. American Journal of Science 31,150

Brinkman, D.B., Sues, H.-D., 1987. A staurikosaurid dinosaur from the Upper Triassic Ischigualasto Formation of Argentina and the relationships of the Staurikosauridae. Palaeontology 30, 493-503.

Broom, R., 1911. On the dinosaurs of the Stormberg, South Africa. Annals. South African Museum 7, 291-308.

Brusatte, S., 2008. Dinosaurs. Quercus Publishing, London. 224 pp.

Brusatte, S.L., Benton, M.J., Ruta, M., Lloyd, G.T., 2008a. Superiority, competition, and opportunism in the evolutionary radiation of dinosaurs. Science 321, 1485-1488.

Brusatte, S.L., Benton, M.J., Ruta, M., Lloyd, G.T., 2008b. The first 50 mya of dinosaur evolution: macroevolutionary pattern and morphological disparity. Biology Letters $4,733-736$.

Brusatte, S., Benton, M., Ruta, M., Lloyd, T., 2008c. The evolutionary history and higherlevel phylogeny of Archosauria (Tetrapoda: Diapsida). Journal of Vertebrate Paleontology 28 (3 Supplement), 58A.

Brusatte, S.L., Benson, R.B.J., Currie, P.J., Zhao, X.-J., 2010a. The skull of Monolophosaurus jiangi (Dinosauria: Theropoda) and its implications for early theropod phylogeny and evolution. Zoological Journal of the Linnean Society 158, 573-607.

Brusatte, S.L., Benton, M.J., Desojo, J.B., Langer, M.C., 2010b. The higher-level phylogeny of Archosauria (Tetrapoda: Diapsida). Journal of Systematic Palaeontology 8, 3-47.

Buffetaut, E., Suteethorn, V., Cuny, G., Tong, H., Le Loeuff, J., Khansubha, S., Jongautchariyakul, S., 2000. The earliest known sauropod dinosaur. Nature 407, 72-74.
Buffetaut, E., Suteethorn, V., Le Loeuff, J., Cuny, G., Tong, H., Khansubha, S., 2002. The first giant dinosaurs: a large sauropod from the Late Triassic of Thailand. Comptes Rendus Palevol 1, 103-109

Butler, R.J., 2005. The 'fabrosaurid' ornithischian dinosaurs of the upper Elliot Formation (Lower Jurassic) of South Africa and Lesotho. Zoological Journal of the Linnean Society $145,175-218$.

Butler, R.J., 2010. The anatomy of the basal ornithischian dinosaur Eocursor parvus from the lower Elliot Formation (Late Triassic) of South Africa. Zoological Journal of the Linnean Society. doi:10.1111/j.1096-3642.2009.00631.x.

Butler, R.J., Porro, L.B., Heckert, A.B., 2006a. A supposed heterodontosaurid tooth from the Rhaetian of Switzerland and a reassessment of the European Late Triassic record of Ornithischia (Dinosauria). Neues Jahrbuch für Geologie und Paläontologie Monatshefte 10, 613-633.

Butler, R.J., Upchurch, P., Norman, D.B., Parish, J.C., 2006b. A biogeographical analysis of the ornithischian dinosaurs. In: Barrett, P.M., Evans, S.E. (Eds.), Ninth International Symposium on Mesozoic Terrestrial Ecosystems and Biota, Abstracts and Proceedings. The Natural History Museum, London, pp. 13-16.

Butler, R.J., Smith, R.M.H., Norman, D.B., 2007. A primitive ornithischian dinosaur from the Late Triassic of South Africa, and the early evolution and diversification of Ornithischia. Proceedings of the Royal Society of London, Series B 274, 2041-2046.

Butler, R.J., Porro, L.B., Norman, D.B., 2008a. A juvenile skull of the primitive ornithischian Heterodontosaurus tucki from the 'Stormberg' of South Africa. Journal of Vertebrate Paleontology 28, 702-711.

Butler, R.J., Upchurch, P., Norman, D.B., 2008b. The phylogeny of the ornithischian dinosaurs. Journal of Systematic Palaeontology 6, 1-40.

Butler, R.J., Galton, P.M., Porro, L.B., Chiappe, L.M., Henderson, D.M., Erickson, G.M., 2010. Lower limits of ornithischian dinosaur body size inferred from a diminutive new Upper Jurassic heterodontosaurid from North America. Proceedings of the Royal Society of London, Series B 277, 375-381.

Cadbury, D., 2002. Terrible Lizard: the First Dinosaur Hunters and the Birth of a New Science. Henry Holt and Company, USA. 384 pp.

Camp, C.L., 1936. A new type of small theropod dinosaur from the Navajo Sandstone of Arizona. Bulletin of the University of California Department of Geological Sciences 24, 39-65.

Carpenter, K., 1997. A giant coelophysoid (Ceratosauria) theropod from the Upper Triassic of New Mexico, USA. Neues Jahrbuch für Geologie und Paläontologie Abhandlungen 205, 189-208.

Carrano, M.T., 2006. Body-size evolution in the Dinosauria. In: Carrano, M.T., Gaudin, T.J., Blob, R.W., Wible, J.R. (Eds.), Amniote Paleobiology: Perspectives on the Evolution of Mammals, Birds, and Reptiles. University of Chicago Press, Chicago, pp. 225-268.

Carrano, M.T., Hutchinson, J.R., 2002. Pelvic and hindlimb musculature of Tyrannosaurus rex (Dinosauria: Theropoda). Journal of Morphology 253, 207-228.

Carrano, M.T., Sampson, S.D., 2004. A review of coelophysoids (Dinosauria: Theropoda) from the Early Jurassic of Europe, with comments on the late history of the Coelophysoidea. Neues Jahrbuch für Geologie und Paläontologie, Monatshefte 2004, 537-558.

Carrano, M.T., Sampson, S.D., 2008. The phylogeny of Ceratosauria (Dinosauria: Theropoda). Journal of Systematic Palaeontology 6, 183-236.

Carrano, M.T., Sampson, S.D., Forster, C.A., 2002. The osteology of Masiakasaurus knopfleri, a small abelisauroid (Dinosauria: Theropoda) from the Late Cretaceous of Madagascar. Journal of Vertebrate Paleontology 22, 510-534.

Carrano, M.T., Hutchinson, J.R., Sampson, S.D., 2005. New information on Segisaurus halli, a small theropod dinosaur from the Early Jurassic of Arizona. Journal of Vertebrate Paleontology 25, 835-849.

Casamiquela, R.M., 1967. Un nuevo dinosaurio ornitisquio Triásico (Pisanosaurus mertii: Ornithopoda) de la Formación Ischigualasto, Argentina. Ameghiniana 4, 47-64.

Chan, K.M.A., Moore, B.R., 2002. Whole-tree methods for detecting differential diversification rates. Systems Biology 51, 855-865.

Chan, K.M.A., Moore, B.R., 2005. SymmeTREE: whole-tree analysis of differential diversification rates. Bioinformatics 21, 1709-1710.

Charig, A.J., 1972. The evolution of the archosaur pelvis and hindlimb: an explanation in functional terms. In: Joysey, K.A., Kemp, T.S. (Eds.), Studies in Vertebrate Evolution. Oliver and Boyd, Edinburgh, pp. 121-155.

Charig, A.J., 1976a. Order Thecodontia. In: Kuhn, O. (Ed.), Handbuch der Paläoherpetologie. Gustav Fischer, Stuttgart, pp. 7-10.

Charig, A.J., 1976b. "Dinosaur monophyly and a new class of vertebrates": a critical review. In: Cox, C.B., Bellairs, A.d'A. (Eds.), Morphology and Biology of Reptiles. Linnean Society Symposium Series, no. 3. Academic Press, London, pp. 65-104.

Charig, A.J., 1984. Competition between therapsids and archosaurs during the Triassic Period: a review and synthesis of current theories. Symposium of the Zoological Society of London 52, 597-628.

Charig, A.J., 1993. Recently proposed phylogenetic analyses of the Triassic Archosauria: a critical comparison and evaluation, facilitated by a simple technique for the modification of conflicting dendrograms. Paleontologia Lombarda, Nuova serie 2, 45-62.

Charig, A.J., Attridge, J., Crompton, A.W., 1965. On the origin of the sauropods and the classification of the Saurischia. Proceedings of the Linnean Society, London 176 197-221.

Chatterjee, S., 1982. Phylogeny and classification of the thecodontian reptiles. Nature 295, 317-320.

Chatterjee, S., 1984. A new ornithischian dinosaur from the Triassic of North America. Die Naturwissenschaften 71, 630-631.

Chatterjee, S., 1993. Shuvosaurus, a new theropod. National Geographic Research and Exploration 9, 274-285.

Chen, P., Dong, Z., Zhen, S., 1998. An exceptionally well-preserved theropod dinosaur from the Yixian Formation of China. Nature 391, 147-152. 
Ciampaglio, C.N., Kemp, M., McShea, D.W., 2001. Detecting changes in morphospace occupation patterns in the fossil record: characterization and analysis of measures of disparity. Paleobiology 27, 695-715.

Clapham, M.E., Shen, S., Bottjer, D.J., 2009. The double mass extinction revisited: reassessing the severity, selectivity, and causes of the end-Guadalupian biotic crisis (Late Permian). Paleobiology 35, 32-50.

Clark, J.M., Maryańska, T., Barsbold, R., 2004. Therizinosauroidea, In: Weishampel, D.B., Dodson, P., Osmólska, H. (Eds.), The Dinosauria, 2nd edition. University of California Press, Berkeley, CA, pp. 151-164.

Cleveland, D.M., Nordt, L.C., Atchley, S.C., 2008a. Paleosols, trace fossils, and precipitation estimates of the uppermost Triassic strata in northern New Mexico. Palaeogeography, Palaeoclimatology, Palaeoecology 257, 421-444.

Cleveland, D.M., Nordt, L.C., Dworkin, S.I., Atchley, S.C., 2008b. Pedogenic carbonate isotopes as evidence for extreme climatic events preceding the Triassic-Jurassic boundary: implications for the biotic crisis? Geological Society of America Bulletin $120,1408-1415$.

Cohen, A.S., Coe, A.L., 2007. The impact of the Central Atlantic Magmatic Province on climate and on the Sr- and Os-isotope evolution of seawater. Palaeogeography, Palaeoclimatology, Palaeoecology 244, 374-390.

Colbert, E.H., 1964. Relationships of saurischian dinosaurs. American Museum Novitates 2181, 1-24

Colbert, E.H., 1970. A saurischian dinosaur from the Triassic of Brazil. American Museum Novitates 2405, 1-39.

Colbert, E.H., 1981. A primitive ornithischian dinosaur from the Kayenta Formation of Arizona. Bulletin of the Museum of Northern Arizona 53, 1-61.

Colbert, E.H., 1989. The Triassic dinosaur Coelophysis. Bulletin of the Museum of Northern Arizona 57, 1-160.

Colbert, E.H., Baird, D., 1958. Coelurosaur bone casts from the Connecticut Valley Triassic. American Museum Novitates 1901, 1-11.

Colombi, C.E., Parrish, J.T., 2008. Late Triassic environmental evolution in southwestern Pangea: plant taphonomy of the Ischigualasto Formation. Palaios 23, 778-795.

Cope, E.D., 1869. Synopsis of the extinct Batrachia, Reptilia, and Aves of North America. Transactions of the American Philosophical Society 40, 1-252.

Cope, E.D, 1889. On a new genus of Triassic Dinosauria. The American Naturalist 23, 626

Crompton, A.W., Charig, A.J., 1962. A new ornithischian from the Upper Triassic of South Africa. Nature 196, 1074-1077.

Cruickshank, A.R.I., Benton, M.J., 1985. Archosaur ankles and the relationships of the thecodontian and dinosaurian reptiles. Nature 317, 715-717.

Crush, P.J., 1984. A late Upper Triassic sphenosuchid crocodilian from Wales. Palaeontology 27, 131-157.

Cuny, G., Galton, P.M., 1993. Revision of the Airel theropod dinosaur from the TriassicJurassic boundary (Normandy, France). Neues Jahrbuch für Geologie und Paläontologie Abhandlungen 187, 261-288.

Cuny, G., Hunt, A.P., Mazin, J.M., Rauscher, R., 2000. Teeth of enigmatic neoselachian sharks and an ornithischian dinosaur from the uppermost Triassic of Lons-leSaunier (Jura, France). Paläontologishe Zeitscrift 74, 171-185.

Currie, B.S., Colombi, C.E., Tabor, N.J., Shipman, T.C., Montañez, I.P., 2009. Stratigraphy and architecture of the Upper Triassic Ischigualasto Formation, Ischigualasto Provincial Park, San Juan, Argentina. Journal of South American Earth Sciences 27, 74-87.

Dal Sasso, C., 2003. Dinosaurs of Italy. Comptes Rendus Palevol 2, 45-66.

Dalton, R., 2001. Elusive fossil could conceal answer to dinosaur debate. Nature 412, 844

de Queiroz, K., Gauthier, J., 1990. Phylogeny as a central principle in taxonomy: phylogenetic definitions of taxon names. Systematic Zoology 39, 307-322.

de Queiroz, K., Gauthier, J., 1992. Phylogenetic Taxonomy. Annual Review of Ecology and Systematics 23, 449-480.

Dobzhansky, T., Ayala, F.J., Stebbins, G.L., Valentine, J.W., 1977. Evolution. W.H. Freeman, San Francisco.

Dodson, P., 1990. Counting dinosaurs: how many kinds were there? Proceedings of the National Academy of Sciences of the United States of America 87, 7608-7612.

Dong, Z.-M., 2001. Primitive armored dinosaur from the Lufeng Basin, Yunnan. In: Tanke, D.H., Carpenter, K. (Eds.), Mesozoic Vertebrate Life. Indiana University Press, Bloomington, IN, pp. 237-242.

Dubiel, R.F., 1994. Triassic deposystems, paleogeography, and paleoclimate of the Western Interior. In: Caputo, M.V., Peterson, J.A., Franczyk, K.J. (Eds.), Mesozoic Systems of the Rocky Mountain region, USA. Society of Economic Paleontologists and Mineralogists Rocky Mountain Section, Denver, pp. 133-168.

Dubiel, R.F., Parrish, J.T., Parrish, J.M., Good, S.C., 1991. The Pangaean megamonsoon evidence from the Upper Triassic Chinle Formation, Colorado Plateau. Palaios 6, 347-370.

Dunlavey, M.G., Whiteside, J.H., Irmis, R.B., 2009. Ecosystem instability during the rise of dinosaurs: evidence from the Late Triassic in New Mexico and Arizona. Geological Society of America Abstracts with Programs 41, 477.

Dzik, J., 2003. A beaked herbivorous archosaur with dinosaur affinities from the early Late Triassic of Poland. Journal of Vertebrate Paleontology 23, 556-574.

Dzik, J., Sulej, T., 2007. A review of the early Late Triassic Krasiejów biota from Silesia, Poland. Palaeontologia Polonica 64, 3-27.

Dzik, J., Sulej, T., Niedźwiedzki, G., 2008. A dicynodont-theropod association in the latest Triassic of Poland. Acta Palaeontologica Polonica 53, 733-738.

Erwin, D.H., 2006. Extinction: How Life on Earth Nearly Ended 250 Million Years Ago. Princeton University Press, Princeton, NJ. ix + 296 pp.

Erwin, D.H., 2007. Disparity: morphological pattern and developmental context. Palaeontology 50, 57-73

Ezcurra, M.D., 2006. A review of the systematic position of the dinosauriform archosaur Eucoelophysis baldwini Sullivan and Lucas, 1999 from the Upper Triassic of New Mexico, USA. Geodiversitas 28, 649-684.
Ezcurra, M.D., 2007. The cranial anatomy of the coelophysoid theropod Zupaysaurus rougieri from the Upper Triassic of Argentina. Historical Biology 19, 185-202.

Ezcurra, M.D., 2008. A new early dinosaur from the Carnian Ischigualasto Formation (NW Argentina) and the origin of dinosaurs. In: Calvo, J.O., Valieri, R.J., Porfiri, J.D. dos Santos, D. (Eds.), Actas de Resúmenes, III Congreso Latinoamericano de Paleontología de Vertebrados. Universidad Nacional del Comahue, Neuquén, p. 87.

Ezcurra, M.D., Cuny, G., 2007. The coelophysoid Lophostropheus airelensis, gen. nov.: a review of the systematics of "Liliensternus" airelensis from the Triassic-Jurassic outcrops of Normandy (France). Journal of Vertebrate Paleontology 27, 73-86.

Ezcurra, M.D., Novas, F.E., 2007. Phylogenetic relationships of the Triassic theropod Zupaysaurus rougieri from NW Argentina. Historical Biology 19, 35-72.

Fedak, T.J., Galton, P.M., 2007. New information on the braincase and skull of Anchisaurus polyzelus (Lower Jurassic, Connecticut, USA; Saurischia: Sauropodomorpha): implications for sauropodomorph systematics. Special Papers in Palaeontology 77, 245-260.

Ferigolo, J., Langer, M.C., 2007. A Late Triassic dinosauriform from south Brazil and the origin of the ornithischian predentary bone. Historical Biology 19, 23-33.

Flynn, J.J., Parrish, J.M., Rakotosamimanana, B., Simpson, W.F., Whatley, R.L., Wyss, A.R. 1999. A Triassic fauna from Madagascar, including early dinosaurs. Science 286, 763-765.

Flynn, J., Nesbitt, S., Parrish, M., Ranivoharimanana, L., Wyss, A., 2008. A new species of basal archosauromorph from the Late Triassic of Madagascar. Journal of Vertebrate Paleontology 28 (3 Supplement), 78A.

Flynn, J.J., Nesbitt, S.J., Parrish, J.M., Ranivoharimanana, L., Wyss, A.R., 2010. A new species of Azendohsaurus (Diapsida: Archosauromorpha) from the Triassic Isalo Group of southwestern Madagascar: cranium and mandible. Palaeontology 53 (3), 669-688.

Fraas, E., 1913. Die neuesten Dinosaurierfunde der schwäbischen Trias. Naturwissenschaften $45,1097-1100$

Fraser, N.C., Padian, K., Walkden, G.M., Davis, A.L.M., 2002. Basal dinosauriform remains from Britain and the diagnosis of the Dinosauria. Palaeontology 45, 79-95.

Fröbisch, J., 2008. Global taxonomic diversity of anomodonts (Tetrapoda, Therapsida) and the terrestrial rock record across the Permian-Triassic boundary. PLOS ONE 3 (11), e3733.

Furin, S., et al., 2006. High-precision U-Pb zircon age from the Triassic of Italy: implications for the Triassic time scale and the Carnian origin of calcareous nannoplankton and dinosaurs. Geology 34, 1009-1012.

Galton, P.M., 1971. The prosauropod dinosaur Ammosaurus, the crocodile Protosuchus, and their bearing on the age of the Navajo Sandstone of northeastern Arizona. Journal of Paleontology 45, 781-795.

Galton, P.M., 1973. The cheeks of ornithischian dinosaurs. Lethaia 6, 67-89.

Galton, P.M., 1976. Prosauropod dinosaurs (Reptilia: Saurischia) of North America. Postilla 169, 1-98.

Galton, P.M., 1978. Fabrosauridae, the basal family of ornithischian dinosaurs (Reptilia: Ornithopoda). Paläontologishe Zeitscrift 52, 138-159.

Galton, P.M., 1983. The oldest ornithischian dinosaurs in North America from the Late Triassic of Nova Scotia, NC, and PA. Geological Society of America, Abstracts with Programs 15, 122.

Galton, P.M., 1985. Diet of prosauropod dinosaurs from the Late Triassic and Early Jurassic. Lethaia 18, 105-123.

Galton, P.M., 1986. Herbivorous adaptations of Late Triassic and Early Jurassic dinosaurs. In: Padian, K. (Ed.), The Beginning of the Age of Dinosaurs. Cambridge University Press, Cambridge, pp. 203-221.

Galton, P.M., 2000. Are Spondylosoma and Staurikosaurus (Santa Maria Formation, Middle-Upper Triassic, Brazil) the oldest saurischian dinosaurs? Paläontologishe Zeitscrift 74, 393-423.

Galton, P.M., 2001. The prosauropod dinosaur Plateosaurus Meyer, 1837 (Saurischia: Sauropodomorpha; Upper Triassic). II. notes on the referred species. Revue de Paléobiologie 20, 435-502.

Galton, P.M., 2005. Bones of large dinosaurs (Prosauropoda and Stegosauria) from the Rhaetic Bone Bed (Upper Triassic) of Aust Cliff, southwest England. Revue de Paléobiologie 24, 51-74.

Galton, P.M., Upchurch, P., 2004. Prosauropoda, In: Weishampel, D.B., Dodson, P., Osmólska, H. (Eds.), The Dinosauria, 2nd Edition. University of California Press, Berkeley, pp. 232-258.

Galton, P.M., van Heerden, J., 1985. Partial hindlimb of Blikanasaurus cromptoni n. gen. and $\mathrm{n}$. sp., representing a new family of prosauropod dinosaurs from the Upper Triassic of South Africa. Geobios 18, 509-516.

Galton, P.M., Yates, A.M., Kermack, D., 2007. Pantydraco n. gen. for Thecodontosaurus caducus Yates, 2003, a basal sauropodomorph dinosaur from the Upper Triassic or Lower Jurassic of South Wales, UK. Neues Jahrbuch für Geologie und Paläontologie Abhandlungen 243, 119-125.

Garland Jr., T.G., 1992. Rate tests for phenotypic evolution using phylogenetically independent contrasts. The American Naturalist 140, 509-519.

Gascoigne, R.M., 1991. Julian Huxley and biological progress. Journal of the History of Biology 24, 433-455.

Gauthier, J.A., 1986. Saurischian monophyly and the origin of birds. Memoirs of the California Academy of Sciences 8, 1-55.

Gierlinski, G., 1999. Tracks of a large thyreophoran dinosaur from the Early Jurassic of Poland. Acta Palaeontologica Polonica 44, 231-234.

Gingerich, P.D., 1993. Quantification and comparison of evolutionary rates. American Journal of Science 293A, 453-478.

Godefroit, P., Cuny, G., 1997. Archosauriform teeth from the Upper Triassic of SaintNicolas-de-Port (northeastern France). Palaeovertebrata 26, 1-34.

Godefroit, P., Knoll, F., 2003. Late Triassic dinosaur teeth from southern Belgium. Comptes Rendus Palevol 2, 3-11. 
Godefroit, P., Cuny, G., Delsate, D., Roche, M., 1998. Late Triassic vertebrates from Syren (Luxembourg). Neues Jahrbuch für Geologie und Paläontologie Abhandlungen 210 305-343.

Goehlich, U.B., Tischlinger, H., Chiappe, L.M., 2006. Juraventaor starki (Reptilia, Theropoda) ein nuer Raubdinosaurier aus dem Oberjura der Suedlichen Frankenalb (Sueddeutschland): Skelettanatomie und Wiechteilbefunde. Archaeopteryx 24, 1-26.

Gould, S.J., 1989. Wonderful Life: The Burgess Shale and the Nature of History. W.W Norton \& Company, New York, NY.

Gould, S.J., 2002. The Structure of Evolutionary Theory. Harvard University Press, Cambridge, MA.

Gould, S.J., Calloway, C.B., 1980. Clams and brachiopods - ships that pass in the night Paleobiology 6, 383-396.

Gow, C.E., 1975. A new heterodontosaurid from the Red Beds of South Africa showing clear evidence of tooth replacement. Zoological Journal of the Linnean Society 57, 335-339.

Gow, C.E., 1990. A tooth-bearing maxilla referable to Lycorhinus angustidens Haughton, 1924 (Dinosauria, Ornithischia). Annals. South African Museum 99, 367-380.

Gower, D.J., 2003. Osteology of the early archosaurian reptile Erythrosuchus africanus Broom. Annals. South African Museum 110, 1-84.

Hammer, W.R., Hickerson, W.J., 1994. A crested theropod dinosaur from Antarctica. Science 264, 828-830.

Haubold, H., 1990. Ein neuer Dinosaurier (Ornithischia, Thyreophora) aus dem unteren Jura des nördlichen Mitteleuropa. Revue de Paléobiologie 9, 149-177.

Haughton, S.H., 1924. The fauna and stratigraphy of the Stormberg Series. Annals. South African Museum 12, 323-497.

Hautmann, M., Benton, M.J., Tomašových, A., 2008. Catastrophic ocean acidification a the Triassic-Jurassic boundary. Neues Jahrbuch für Geologie und Paläontologie Abhandlungen 249, 119-127.

He, X., et al., 1998. A new sauropod dinosaur from the Early Jurassic in Gongxian County, south Sichuan [in Chinese]. Acta Geologica Sichuan 18, 1-6.

Heckert, A.B., 2002. A revision of the Upper Triassic ornithischian dinosaur Revueltosaurus, with a description of a new species. New Mexico Museum of Natural History and Science, Bulletin 21, 253-268.

Heckert, A.B., 2004. Late Triassic microvertebrates from the lower Chinle Group (Otischalkian-Adamanian: Carnian), southwestern U.S.A. New Mexico Museum of Natural History and Science, Bulletin 27, 1-170.

Heckert, A.B., 2005. Kryzanowskisaurus, a new name for a probable ornithischian dinosaur from the Upper Triassic Chinle Group, Arizona and New Mexico, USA. In: Heckert, A.B., Lucas, S.G. (Eds.) Vertebrate Paleontology in Arizona. New Mexico Museum of Natural History \& Science, Albuquerque, NM, pp. 77-83.

Heckert, A.B., Lucas, S.G., 1995. Synchronous Pangea-wide diversification of Late Triassic dinosaurs and the importance of western North America in early dinosaur evolution. Geological Society of America Abstracts with Programs 27, 319.

Heckert, A.B., Harris, J.D., Lucas, S.G., Zeigler, K.E., 2000. Additions to the fauna of the Upper Triassic Snyder Quarry, Petrified Forest Formation (Revueltian: early-mid Norian), north-central New Mexico. New Mexico Geology 22, 46.

Heckert, A.B., Zeigler, K.E., Lucas, S.G., Rinehart, L.F., 2003. Coelophysids (Dinosauria: Theropoda) from the Upper Triassic (Revueltian) Snyder Ouarry. New Mexico Museum of Natural History and Science Bulletin 24, 127-132.

Hitchcock, E., 1865. A Supplement to the Ichnology of New England. Wright and Potter Boston. 96 pp.

Holtz, T.R., Molnar, R.E., Currie, P.J., 2004. Basal Tetanurae, In: Weishampel, D.B., Dodson, P., Osmólska, H. (Eds.), The Dinosauria, 2nd edition. University of California Press, Berkeley, CA, pp. 71-110.

Hopson, J.A., 1975. On the generic separation of the ornithischian dinosaurs Lycorhinus and Heterodontosaurus from the Stormberg Series (Upper Triassic of South Africa). South African Journal of Science 71, 302-305.

Hornung, T., et al., 2007. Multistratigraphic constraints on the NE Tethyan "Carnian crisis". In: Lucas, S.G., Spielmann, J.A. (Eds.), The Global Triassic, New Mexico Museum of Natural History and Science Bulletin, vol. 41, pp. 59-67.

Hu, S., 1993. A new Theropoda (Dilophosaurus sinensis sp. nov.) from Yunnan, China Vertebrata PalAsiatica 31, 65-69.

Hunt, A.P., 1989. A new ?ornithischian dinosaur from the Bull Canyon Formation (Upper Triassic) of east central New Mexico. In: Lucas, S.G., Hunt, A.P. (Eds.), The Dawn of the Age of Dinosaurs in the American Southwest. New Mexico Museum of Natural History \& Science, Albuquerque, NM, pp. 355-358.

Hunt, A.P., 1991. The early diversification pattern of dinosaurs in the Late Triassic. Modern Geologist 16, 43-60.

Hunt, A.P., 1996. A new clade of herrerasaur-like theropods from the Late Triassic of western North America. Journal of Vertebrate Paleontology 16 (supplement to 3), $43 \mathrm{~A}$

Hunt, A.P., Lucas, S.G., 1994. Ornithischian dinosaurs from the Upper Triassic of the United States. In: Fraster, N.C., Sues, H.-D. (Eds.), In the Shadow of the Dinosaurs: Early Mesozoic Tetrapods. Cambridge University Press, Cambridge, pp. 227-241.

Hunt, A.P., Sullivan, R.M., Lucas, S.G., Heckert, A., 1995. Herrerasaur and theropod diversity in the Late Triassic of western United States. Journal of Vertebrate Paleontology 15 (3 Supplement), 36A.

Hunt, A.P., Lucas, S.G., Heckert, A.B., Sullivan, R.M., Lockley, M.G., 1998. Late Triassic dinosaurs from the western United States. Geobios 31, 511-531.

Huxley, T.H., 1868. On the animals which are most nearly intermediate between the birds and reptiles. Geological Magazine 5, 357-365.

Huxley, T.H., 1870a. Further evidence of the affinity between the dinosaurian reptiles and birds. Quarterly Journal of the Geological Society of London 26, 12-31.

Huxley, T.H., 1870b. On the classification of the Dinosauria with observations on the Dinosauria of the Trias. I. The classification and affinities of the Dinosauria. Quarterly Journal of the Geological Society of London 26, 32-38.
Irmis, R.B., 2004. First report of Megapnosaurus (Theropoda: Coelophysoidea) from China. PaleoBios 24, 11-18.

Irmis, R.B., 2005. A review of the vertebrate fauna of the Lower Jurassic Navajo Sandstone in Arizona. Mesa Southwest Museum Bulletin 11, 55-71.

Irmis, R.B., 2009. Evaluating Late Triassic faunal change and the rise of dinosaurs: examples from the Chinle Formation of north-central New Mexico. Geological Society of America Abstracts with Programs 41 (7), 159.

Irmis, R.B., Knoll, F., 2008. New ornithischian dinosaur material from the Lower Jurassic Lufeng Formation of China. Neues Jahrbuch für Geologie und Paläontologie Abhandlungen 247, 117-128.

Irmis, R., Mundil, R., 2008. New age constraints from the Chinle Formation revise global comparisons of Late Triassic vertebrate assemblages. Journal of Vertebrate Paleontology 28 (3 Supplement), 95A.

Irmis, R.B., et al., 2007a. A Late Triassic dinosauromorph assemblage from New Mexico and the rise of dinosaurs. Science 317, 358-361.

Irmis, R.B., Parker, W.G., Nesbitt, S.J., Liu, J., 2007b. Early ornithischian dinosaurs: the Triassic record. Historical Biology 19, 3-22.

Jablonski, D., 1986. Background and mass extinctions: the alternation of macroevolutionary regimes. Science 231, 129-133.

Jablonski, D., 1991. Extinctions: a paleontological perspective. Science 253, 754-757.

Jablonski, D., 2001. Lessons from the past: evolutionary impacts of mass extinctions. Proceedings of the National Academy of Sciences of the United States of America 98, 5393-5398.

Jablonski, D., 2005. Mass extinctions and macroevolution. Paleobiology 31, 192-210.

Jablonski, D., 2008. Extinction and the spatial dynamics of biodiversity. Proceedings of the National Academy of Sciences of the United States of America 105, 11528-11535.

Jain, S.L., Kutty, T.S., Roy Chowdhury, T., Chatterjee, S., 1975. The sauropod dinosaur from the Lower Jurassic Kota Formation of India. Proceedings of the Royal Society of London, Series A 188, 221-228.

Jenkins Jr., F.A., et al., 1994. Late Triassic continental vertebrates and depositional environments of the Fleming Fjord Formation, Jameson Land, east Greenland. Meddelelser om Gronland. Geoscience 32, 1-25.

Jenny, J., Jenny-Deshusses, C., Marrec, A.L., Taquet, P., 1980. Découverte d'ossements de dinosauriens dans le Jurassique inférieur (Toarcien) du Haut Atlas central (Maroc). Comptes Rendus de l'Académie des Sciences, Paris, D 290, 839-842.

Ji, Q., Ji, S.-A., 1996. On discovery of the earliest bird fossil in China and the origin of birds. Chinese Geology 10, 30-33.

Ji, Q., Yuan, C., 2002. Discovery of two types of protofeathered pterosaurs in the Mesozoic Daohuguo biota in the Nincheng region and its stratigraphic and biologic significances. Geological Review 48, 221-224.

Ji, Q., Currie, P.J., Norell, M.A., Ji, S.-A., 1998. Two feathered dinosaurs from northeastern China. Nature 393, 753-761.

Ji, Q., Norell, M.A., Gao, K.-Q., Ji, S.-A., Ren, D., 2001. The distribution of integumentary structures in a feathered dinosaur. Nature 410, 1084-1087.

Ji, Q., Ji, S., Lu, J., You, H., Chen, W., Liu, Y., Liu, Y., 2005. First avialan bird from China (Jinfengopteryx elegans gen. et sp. nov.). Geological Bulletin of China 24, 197-205.

Ji, S.-A., Ji, Q., Lu, J., Yuan, C., 2007. A new giant compsognathid dinosaur with long filamentous integuments from Lower Cretaceous of Northeastern China. Acta Geologica Sinica 81, 8-15.

Jones, K.E., Bininda-Emonds, O.R.P., Gittleman, J.L., 2005. Bats, clocks, and rocks: diversification patterns in Chrioptera. Evolution 59, 2243-2255.

Jourdan, F., Marzoli, A., Bertrand, H., Cirilli, S., Tanner, L.H., Kontak, D.J., McHone, G. Renne, P.R., Bellieni, G., 2009. ${ }^{40} \mathrm{Ar} /{ }^{39} \mathrm{Ar}$ ages of CAMP in North America: implications for the Triassic-Jurassic boundary and the ${ }^{40} \mathrm{~K}$ decay constant bias. Lithos 110, 167-180.

Juul, L., 1994. The phylogeny of basal archosaurs. Palaeontologia Africana 31, 1-38.

Kellner, A.W.A., Wang, X., Tischlinger, H., Campos, D.A., Hone, D.W.E., Meng, X., 2009. The soft tissue of Jeholopterus (Pterosauria, Anurognathidae, Batrachognathidae) and the structure of the pterosaur wing membrane. Proceedings of the Royal Society of London, Series B 277, 321-329.

Kent, D.V., Olsen, P.E., 1999. Astronomically tuned geomagnetic polarity timescale for the Late Triassic. Journal of Geophysical Research B 104 (B6), 12831-12841.

Kent, D.V., Tauxe, L., 2005. Corrected Late Triassic latitudes for continents adjacent to the North Atlantic. Science 307, 240-244.

Kirby, R.E., 1991. A vertebrate fauna from the Upper Triassic Owl Rock Member of the Chinle Formation of northern Arizona. M.S. Thesis, Northern Arizona University, Flagstaff, p. 476

Knoll, F., 2004. Review of the tetrapod fauna of the "Lower Stormberg Group" of the main Karoo Basin (southern Africa): implication for the age of the lower Elliot Formation. Bulletin de la Société Géologique de France 175, 73-83.

Knoll, F., 2005. The tetrapod fauna of the upper Elliot and Clarens formations in the main Karoo Basin (South Africa and Lesotho). Bulletin de la Société Géologique de France 176, 81-91

Knoll, F., 2008. On the Procompsognathus postcranium (Late Triassic, Germany) Geobios 6, 779-786.

Knoll, F., in press. A primitive sauropodomorph from the upper Elliot Formation of Lesotho. Geological Magazine. doi:10.1017/S001675681000018X.

Kubo, T., Benton, M.J., 2009. Tetrapod postural shift estimated from Permian and Triassic trackways. Palaeontology 52, 1029-1037.

Kuiper, K.F., et al., 2008. Synchronizing rock clocks of earth history. Science 320, 500-504.

Kutty, T.S., Chatterjee, S., Galton, P.M., Upchurch, P., 2007. Basal sauropodomorphs (Dinosauria: Saurischia) from the Lower Jurassic of India: their anatomy and relationships. Journal of Paleontology 81, 1218-1240.

Langer, M.C., 2003. The pelvic and hind limb anatomy of the stem-sauropodomorph Saturnalia tupiniquim (Late Triassic, Brazil). PaleoBios 23, 1-40. 
Langer, M.C., 2004. Basal Saurischia, In: Weishampel, D.B., Dodson, P., Osmólska, H. (Eds.), The Dinosauria, 2nd edition. University of California Press, Berkeley, CA, pp. 25-46.

Langer, M.C., 2005. Studies on continental Late Triassic tetrapod biochronology. II. The Ischigualastian and a Carnian global correlation. Journal of South American Earth Sciences 19, 219-239.

Langer, M.C., Benton, M.J., 2006. Early dinosaurs: a phylogenetic study. Journal of Systematic Palaeontology 4, 309-358.

Langer, M.C., Abdala, F., Richter, M., Benton, M.J., 1999. A sauropodomorph dinosaur from the Upper Triassic (Carnian) of southern Brazil. Comptes Rendus de l'Académie des Sciences, Sciences de la Terre et des Planètes 329, 511-517.

Langer, M.C., Bittencourt, J., Schultz, C., 2007a. The inclusivity and phylogenetic position of Guaibasaurus candelariensis: a basal dinosaur from the Late Triassic of Brazil. Journal of Vertebrate Paleontology 27 (3), 103A.

Langer, M.C., França, M.A.G., Gabriel, S., 2007b. The pectoral girdle and forelimb anatomy of the stem-sauropodomorph Saturnalia tupiniquim (Upper Triassic, Brazil). Special Papers in Palaeontology 77, 113-137.

Langer, M.C., Ribeiro, A.M., Schultz, C.L., Ferigolo, J., 2007c. The continental tetrapodbearing Triassic of south Brazil. New Mexico Museum of Natural History and Science Bulletin 41, 201-218.

Langer, M.C., Ezcurra, M.D., Bittencourt, J.S., Novas, F.E., 2010. The origin and early evolution of dinosaurs. Biological Review 85, 55-110.

Lavin, M., Herendeen, P.S., Wojciechowski, M.F., 2005. Evolutionary rates analysis of Leguminosae implicates a rapid diversification of lineages during the Tertiary. Systems Biology 54, 575-594.

Leal, L.A., Azevedo, S.A.K., Kellner, A.W.A., Da Rosa, Á.A.S., 2004. A new early dinosaur (Sauropodomorpha) from the Caturrita Formation (Late Triassic), Paraná Basin, Brazil. Zootaxa 690, 1-24.

Li, Q., Gao, K.-Q., Vinther, J., Shawkey, M.D., Clarke, J.A., D'Alba, L., Meng, Q., Briggs, D.E.G., Prum, R.O., 2010. Plumage color patterns of an extinct dinosaur. Science 327, 1369-1372.

Litwin, R.J., Traverse, A., Ash, S.R., 1991. Preliminary palynological zonation of the Chinle Formation, southwestern U.S.A., and its correlation to the Newark Supergroup (eastern U.S.A.). Review of Palaeobotany and Palynology 68, 269-287.

Liu, Y., Liu, Y., Zhang, H., 2006. LA-ICPMS zircon U-Pb dating in the Jurassic Daohugou Beds and correlative strata in Ningcheng of Inner Mongolia. Acta Geologica Sinica $80,733-742$

Lloyd, G.T., et al., 2008. Dinosaurs and the Cretaceous Terrestrial Revolution. Proceedings of the Royal Society of London, Series B 274, 2483-2490.

Long, J.A., 2003. The Dinosaur Dealers: Mission: To Uncover International Fossil Smuggling. Allen and Unwin, Sydney. 240 pp.

Long, R.A., Murry, P.A., 1995. Late Triassic (Carnian and Norian) tetrapods from the southwestern United States. Bulletin of the New Mexico Museum of Natural History and Science 4, 1-254.

Lu, J.-C., 2002. Soft tissue in an Early Cretaceous pterosaur from Liaoning Province, China. Memoirs of the Fukui Prefectural Museum 1, 19-28.

Lucas, S.G., 1998. Global Triassic tetrapod biostratigraphy and biochronology. Palaeogeography, Palaeoclimatology, Palaeoecology 143, 347-384.

Luo, Z., Wu, X.-C., 1994. The small tetrapods of the Lower Lufeng Formation, Yunnan, China. In: Fraser, N.C., Sues, H.-D. (Eds.), In the Shadow of the Dinosaurs: Early Mesozoic Tetrapods. Cambridge University Press, Cambridge, pp. 251-270.

MacArthur, R., Wilson, E.O., 1967. The Theory of Island Biogeography. Princeton University Press, Princetion, NJ. 203 pp.

Martínez, R.N., 2009. Adeopapposaurus mognai, gen. et sp. nov. (Dinosauria: Sauropodomorpha), with comments on adaptations of basal Sauropodomorpha. Journal of Vertebrate Paleontology 29, 142-164.

Martinez, R.N., Alcober, O.A., 2009. A basal sauropodomorph (Dinosauria: Saurischia) from the Ischigualasto Formation (Triassic, Carnian) and the early evolution of Sauropodomorpha. PLoS ONE 4 (2), e4397.

Martz, J.W., Small, B.J., 2006. Tecovasuchus chatterjeei, a new aetosaur (Archosauria: Stagonolepididae) from the Tecovas Formation (Carnian, Upper Triassic) of Texas. Journal of Vertebrate Paleontology 26, 308-320.

Mayr, G., Peters, D.S., Plodowski, G., Vogel, O., 2002. Bristle-like integumentary structures at the tail of the horned dinosaur Psittacosaurus. Naturwissenschaften 89, 361-365.

McElwain, J.C., Beerling, D.J., Woodward, F.I., 1999. Fossil plants and global warming at the Triassic-Jurassic boundary. Science 285, 1386-1390.

McGhee Jr., G.R., 1999. Theoretical morphology. The Concept and its Applications. Columbia University Press, New York, NY. 316 pp.

Michalík, J., Lintnerová, O., Gaździcki, A., Soták, J., 2007. Record of environmental changes in the Triassic-Jurassic boundary interval in the Zliechov Basin, western Carpathians. Palaeogeography, Palaeoclimatology, Palaeoecology 244, 71-88.

Mietto, P., 1985. Piste di dinosauri nella Dolomia Principale (Triassico superiore) del Monte Pelmetto (Cadore). Memorie della Società Geoligica Italiana 30, 307-310.

Milàn, J., Gierlinski, G., 2004. A probable thyreophoran (Dinosauria, Ornithischia) footprint from the Upper Triassic of southern Sweden. Bulletin. Geological Society of Denmark 51, 71-75.

Min, K., Mundil, R., Renne, P.R., Ludwig, K.R., 2000. A test for systematic errors in ${ }^{40} \mathrm{Ar} /$ ${ }^{39} \mathrm{Ar}$ geochronology through comparison with $\mathrm{U} / \mathrm{Pb}$ analysis of a $1.1-\mathrm{Ga}$ rhyolite. Geochimica et Cosmochimica Acta 64, 73-98.

Mindell, D.P., Thacker, C.E., 1996. Rates of molecular evolution: phylogenetic issues and applications. Annual Review of Ecology and Systematics 27, 279-303.

Moore, K.A., 2002. Coupled organic and carbonate isotope analysis of Late Triassic paleosols, Ischigualasto Basin, NW Argentina. M.S. Thesis, University of California, Davis, 105 pp.

Mundil, R., 2007. Critical view of the calibration of the Triassic time scale. New Mexico Museum of Natural History and Science Bulletin 41, 314-315.
Mundil, R., Brack, P., Meier, M., Rieber, H., Oberli, F., 1996. High resolution U-Pb dating of Middle Triassic volcaniclastics: time-scale calibration and verification of tuning parameters for carbonate sedimentation. Earth and Planetary Science Letters 141, 137-151.

Mundil, R., et al., 2003. Cyclicities in Triassic platform carbonates: synchronizing radioisotopic and orbital clocks. Terra Nova $15,81-87$.

Mundil, R., Renne, P.R., Min, K.K., Ludwig, K.R., 2006. Resolvable miscalibration of the ${ }^{40} \mathrm{Ar} /{ }^{39} \mathrm{Ar}$ geochronometer. Eos Transactions AGU 87 (52, Fall Meeting Supplement), F2218.

Mundil, R. Gehrels, G., Deino, A.L., Irmis, R.B., 2008. Zircon U-Pb analyses by TIMS and LA-ICPMS on the same material. Eos Transactions AGU 89 (53, Fall Meeting Supplement) abstract V13A-2108.

Muttoni, G., Kent, D.V., Brack, P., Nicora, A., Balini, M., 1997. Middle Triassic magnetostratigraphy and biostratigraphy from the Dolomites and Greece. Earth and Planetary Science Letters 146, 107-120.

Muttoni, G., et al., 2004. Tethyan magnetostratigraphy from Pizzo Mondello (Sicily) and correlation to the Late Triassic Newark astrochronological polarity time scale. Geological Society of America Bulletin 116, 1043-1058.

Muttoni, G., et al., 2010. Rhaetian magneto-biostratigraphy from the southern Alps (Italy): constraints on Triassic chronology. Palaeogeography, Palaeoclimatology, Palaeoecology 285, 1-16.

Nee, S., 2006. Birth-death models in macroevolution. Annual Review of Ecology, Evolution, and Systematics 37, 1-17.

Nesbitt, S.J., 2003. Arizonasaurus and its implications for archosaur divergence. Proceedings of the Royal Society of London, Series B 270, S234-S237.

Nesbitt, S.J., 2005. The osteology of the pseudosuchian Arizonasaurus babbitti. Historical Biology 17, 19-47.

Nesbitt, S.J., 2007. The anatomy of Effigia okeeffeae (Archosauria, Suchia), theropod-Like convergence, and the distribution of related taxa. Bulletin of the American Museum of Natural History 302, 1-84.

Nesbitt, S., 2008. The evolution of the sacrum: identifying homologous sacral vertebrae across Amniota. Journal of Vertebrate Paleontology 28 (3 Supplement), 120A.

Nesbitt, S.J., Norell, M.A., 2006. Extreme convergence in the body plans of an early suchian (Archosauria) and ornithomimid dinosaurs (Theropoda). Proceedings of the Royal Society of London, Series B 273, 1045-1048.

Nesbitt, S.J., Turner, A.H., Erickson, G.M., Norell, M.A., 2006. Prey choice and cannibalistic behaviour in the theropod Coelophysis. Biology Letters 2, 611-614.

Nesbitt, S.J., Irmis, R.B., Parker, W.G., 2007. A critical re-evaluation of the Late Triassic dinosaur taxa of North America. Journal of Systematic Palaeontology 5, 209-243.

Nesbitt, S.J., et al., 2009a. Hindlimb osteology and distribution of basal dinosauromorphs from the Late Triassic of North America. Journal of Vertebrate Paleontology 29, 498-516.

Nesbitt, S.J., et al., 2009b. A complete skeleton of a Late Triassic saurischian and the early evolution of dinosaurs. Science 326, 1530-1533.

Nesbitt, S.J., et al., 2010. Ecologically distinct dinosaurian sister group shows early diversification of Ornithodira. Nature 464, 95-98.

Newman, B.H., 1968. The Jurassic dinosaur Scelidosaurus harrisoni, Owen. Palaeontology $11,40-43$.

Norell, M.A., 1992. Taxic origin and temporal diversity: the effect of phylogeny. In: Novacek, M.J., Wheeler, Q.D. (Eds.), Extinction and Phylogeny. Columbia University Press, New York, NY, pp. 89-118.

Norell, M.A., 1993. Tree-based approaches to understanding history; comments on ranks, rules and the quality of the fossil record. American Journal of Science 293, 407-417.

Norell, M.A., Ellison, M., 2005. Unearthing the Dragon. Pi Press, New York. 224 pp.

Norman, D.B., Witmer, L.M., Weishampel, D.B., 2004a. Basal Ornithischia, In: Weishampel, D.B., Dodson, P., Osmólska, H. (Eds.), The Dinosauria, 2nd edition. University of California Press, Berkeley, CA, pp. 325-334.

Norman, D.B., Witmer, L.M., Weishampel, D.B., 2004b. Basal Thyreophora, In: Weishampel, D.B., Dodson, P., Osmólska, H. (Eds.), The Dinosauria, 2nd edition. University of California Press, Berkeley, CA, pp. 335-342.

Norman, D.B., Sues, H.-D., Witmer, L.M., Coria, R.A., 2004c. Basal Ornithopoda, In: Weishampel, D.B., Dodson, P., Osmólska, H. (Eds.), The Dinosauria, 2nd edition. University of California Press, Berkeley, CA, pp. 393-412.

Norman, D.B., Butler, R.J., Maidment, S.C.H., 2007. Reconsidering the status and affinities of the ornithischian dinosaur Tatisaurus oehleri Simmons, 1965. Zoological Journa of the Linnean Society $150,865-874$.

Novas, F.E., 1989. The tibia and tarsus in the Herrerasauridae (Dinosauria, incertae sedis) and the origin and evolution of the dinosaurian tarsus. Journal of Paleontology 63, 677-690.

Novas, F.E., 1992. Phylogenetic relationships of the basal dinosaurs, the Herrerasauridae. Palaeontology 16, 51-62.

Novas, F.E., 1993. New information on the systematics and postcranial skeleton of Herrerasaurus iscshigualastensis (Theropoda: Herrerasauridae) from the Ischigualasto Formation (Upper Triassic) of Argentina. Journal of Vertebrate Paleontology 13, 400-423.

Novas, F.E., 1996. Dinosaur monophyly. Journal of Vertebrate Paleontology 16, 723-741.

O'Connor, P.M., 2007. The postcranial axial skeleton of Majungasaurus crenatissimus (Theropoda: Abelisauridae) from the Late Cretaceous of Madagascar. In: Sampson, S.D., Krause, D.W. (Eds.), Majungasaurus crenatissimus (Theropoda: Abelisauridae) from the Late Cretaceous of Madagascar: Society of Vertebrate Paleontology Memoir, vol. 8, pp. 127-162

O'Meara, B.C., Ané, C., Sanderson, M.J., Wainwright, P.C., 2006. Testing for different rates of continuous trait evolution using likelihood. Evolution 60, 922-933.

Oakley, T.H., 2003. Maximum likelihood models of trait evolution. Comments on Theoretical Biology 8, 1-17. 
Olsen, P.E., Baird, D., 1986. The ichnogenus Atreipus and its significance for Triassic biostratigraphy. In: Padian, K. (Ed.), The Beginning of the Age of Dinosaurs. Cambridge University Press, Cambridge, pp. 61-87.

Olsen, P.E., Galton, P.M., 1984. A review of the reptile and amphibian assemblages from the Stormberg Group of southern Africa with special emphasis on the footprints and the age of the Stormberg. Palaeontologica Africana 25, 87-110.

Olsen, P.E., Huber, P., 1998. The oldest Late Triassic footprint assemblage from North America (Pekin Formation, Deep River Basin, North Carolina, USA). Southeastern Geology 38, 77-90.

Olsen, P.E., Rainforth, E.C. 2003. The Early Jurassic ornithischian dinosaurian ichnogenus Anomoepus. In: LeTourneau, P.M., Olsen, P.E. (Eds.), The Great Rift Valleys of Pangea in Eastern North America. : Sedimentology, Stratigraphy, and Paleontology, Volume 2. Columbia University Press, New York, pp. 314-368.

Olsen, P.E., et al., 2002. Ascent of dinosaurs linked to an iridium anomaly at the TriassicJurassic boundary. Science 296, 1305-1307.

Ostrom, J.H., 1969. A new theropod dinosaur from the Lower Cretaceous of Montana. Postilla 128, 1-17.

Ostrom, J.H., 1973. The ancestry of birds. Nature 242, 136

Owen, R., 1842. Report on British Fossil Reptiles. Part II. Reports of the British Association for the Advancement of Science 11, 60-204.

Owen, R., 1854. Descriptive Catalogue of the Fossil Organic Remains of Reptilia and Pices Contained in the Museum of the Royal College of Surgeons of England. Taylor and Francis, London. 184 pp.

Owen, R., 1861. Monograph on the fossil Reptilia of the Liassic formations. Part 1. A monograph of a fossil dinosaur (Scelidosaurus harrisonii Owen) of the Lower Lias. Palaeontological Society Monograph 13, 1-14.

Owen, R., 1863. A monograph on the Fossil Reptilia of the Liassic formations. Part 2. A monograph of a fossil dinosaur (Scelidosaurus harrisonii Owen) of the Lower Lias. Palaeontological Society Monograph 2, 1-26.

Padian, K., 1986. Introduction. In: Padian, K. (Ed.), The Beginning of the Age of Dinosaurs. Cambridge University Press, Cambridge, pp. 1-7.

Padian, K., 1989. Presence of the dinosaur Scelidosaurus indicates Jurassic age for the Kayenta Formation (Glen Canyon Group, northern Arizona). Geology 17, 438-441.

Padian, K., 1990. The ornithischian form genus Revueltosaurus from the Petrified Fores of Arizona (Late Triassic: Norian; Chinle Formation). Journal of Vertebrate Paleontology 10, 268-269.

Padian, K., Chiappe, L.M., 1998. The origin and early evolution of birds. Biological Review 73, 1-42.

Padian, K., May, C.L., 1993. The earliest dinosaurs. In: Lucas, S.G., Morales, M. (Eds.), The Nonmarine Triassic: New Mexico Museum of Natural History and Science Bulletin, vol. 3, pp. 379-380.

Pagel, M., 1998. Inferring evolutionary process from phylogenies. Zoologica Scripta 26, 331-348.

Parker, W.G., Irmis, R.B., Nesbitt, S.J., Martz, J.W., Browne, L.S., 2005. The Late Triassic pseudosuchian Revueltosaurus callenderi and its implications for the diversity of early ornithischian dinosaurs. Proceedings of the Royal Society of London, Series B 272, 963-969.

Parrish, J.T., 1993. Climate of the supercontinent Pangea. Journal of Geology 101, 215-233.

Peczkis, J., 1994. Implications of body-mass estimates for dinosaurs. Journal of Vertebrate Paleontology 14, 520-533.

Pol, D., Powell, J.E., 2007a. New information on Lessemsaurus sauropoides (Dinosauria: Sauropodomorpha) from the Upper Triassic of Argentina. Special Papers in Palaeontology 77, 223-243.

Pol, D., Powell, J.E., 2007b. Skull anatomy of Mussaurus patagonicus (Dinosauria: Sauropodomorpha) from the Late Triassic of Patagonia. Historical Biology 19, 125-144.

Prochnow, S.J., Nordt, L.C., Atchley, S.C., Hudec, M.R., 2006. Multi-proxy paleosol evidence for Middle and Late Triassic climate trends in eastern Utah. Palaeogeography, Palaeoclimatology, Palaeoecology 232, 53-72.

Purvis, A., 2008. Phylogenetic approaches to the study of extinction. Annual Review of Ecology, Evolution, and Systematics 39, 301-319.

Raath, M.A., 1969. A new coelurosaurian dinosaur from the Forest Sandstone of Rhodesia. Arnoldia 4, 1-25.

Raath, M.A., 1972. Fossil vertebrate studies in Rhodesia: a new dinosaur (Reptilia: Saurischia) from near the Trias-Jurassic boundary. Arnoldia 5, 1-37.

Rauhut, O.W.M., 2003. The interrelationships and evolution of basal theropod dinosaurs. Special Papers in Palaeontology 69, 1-213.

Rauhut, O.W.M., Hungerbühler, A., 2000. A review of European Triassic theropods. Gaia $15,75-88$.

Raup, D.M., 1965. Theoretical morphology of the coiled shell. Science 147, 1294-1295.

Raup, D.M., 1979. Size of the Permo-Triassic bottleneck and its evolutionary implications. Science 206, 217-218.

Raup, D.M., 1986. Biological extinction in Earth history. Science 231, 1528-1533.

Rayfield, E.J., 2005. Aspects of comparative cranial mechanics in the theropod dinosaurs Coelophysis, Allosaurus and Tyrannosaurus. Zoological Journal of the Linnean Society 144, 309-316. 144, 309-316.

Rayfield, E.J., Barrett, P.M., McDonnell, R.A., Willis, K.J., 2005. A geographica information system (GIS) study of Triassic vertebrate biochronology. Geological Magazine 142, 327-354

Rayfield, E.J., Barrett, P.M., Milner, A.R., 2009. Utility and validity of Middle and Late Triassic "land vertebrate faunachrons.". Journal of Vertebrate Paleontology 29, $80-87$.

Reig, O.A., 1963. La presencia de dinosaurios saurisquios en los "Estratos de Ischigualasto" (Mesotriásico superior) de las Provincias de San Juan y La Rioja (Republica Argentina). Ameghiniana 3, 3-20.
Ricklefs, R.E., 2007. Estimating diversification rates from phylogenetic information. Trends in Ecology and Evolution 22, 601-610.

Riggs, N.R., Ash, S.R., Barth, A.P., Gehrels, G.E., Wooden, J.L., 2003. Isotopic age of the Black Forest Bed, Petrified Forest Member, Chinle Formation, Arizona: an example of dating a continental sandstone. Geological Society of America Bulletin 115, 1315-1323.

Riley, H., Stutchbury, S., 1836. A description of various fossil remains of three distinct saurian animals discovered in the autumn of 1834, in the Magnesian Conglomerate on Durdham Down, near Bristol. Proceedings of the Geological Society of London 2 397-399.

Rinehart, LF, Lucas, S.L. Heckert, A.B., Spielmann, J.A., Celeskey, M.D., 2009. The paleobiology of Coelophysis bauri (Cope) from the Upper Triassic (Apachean) Whitaker quarry, New Mexico, with detailed analysis of a single quarry block. New Mexico Museum of Natural History and Science Bulletin 45, 1-260.

Robinson, P.L., 1971. A problem of faunal replacement on Permo-Triassic continents. Palaeontology 14, 131-153.

Rogers, R.R., et al., 1993. The Ischigualasto tetrapod assemblage (Late Triassic Argentina) and ${ }^{40} \mathrm{Ar} /{ }^{39} \mathrm{Ar}$ dating of dinosaur origins. Science 260, 794-797.

Rogers, R.R., et al., 2001. Paleoenvironment and taphonomy of the Chañares Formation tetrapod assemblage (Middle Triassic), northwestern Argentina: spectacular preservation in volcanogenic concretions. Palaios 16, 461-481.

Romer, A.S., 1966. Vertebrate Paleontology, 3rd ed. Univ. of Chicago Press, Chicago, IL.

Roopnarine, P.D., 2003. Analysis of rates of morphologic evolution. Annual Review of Ecology, Evolution, and Systematics 34, 605-632.

Rosenbaum, J.N., Padian, K., 2000. New material of the basal thyreophoran Scutellosaurus lawleri from the Kayenta Formation (Lower Jurassic) of Arizona. PaleoBios 20, 13-23.

Rowe, T., 1989. A new species of the theropod dinosaur Syntarsus from the Early Jurassic Kayenta Formation of Arizona. Journal of Vertebrate Paleontology 9, 125-136.

Roy, J., 1996. The roles of mass extinction and biotic interaction in large-scale replacements: a reexamination using the fossil record of stromboidean gastropods. Paleobiology 22, 436-452.

Ruta, M., Benton, M.J., 2008. Calibrated diversity, tree topology and the mother of mass extinctions: the lesson of temnospondyls. Palaeontology 51, 1261-1288.

Ruta, M., Wagner, P.J., Coates, M.I., 2006. Evolutionary patterns in early tetrapods. I. Rapid initial diversification followed by decrease in rates of character change. Proceedings of the Royal Society of London, Series B 273, 2107-2111.

Rutimeyer, L., 1856. Reptilienknochen aus dem Keuper. Allgemeinen Schweizerischen Gesellschaft fur die Gesammten Naturwissenschaften, Verhandlungen 41,62-64.

Sahney, S., Benton, M.J., 2008. Recovery from the most profound mass extinction of all time. Proceedings of the Royal Society of London, Series B 275, 759-765.

Santa Luca, A.P., 1980. The postcranial skeleton of Heterodontosaurus tucki (Reptilia Ornithischia) from the Stormberg of South Africa. Annals. South African Museum 79, 159-211.

Santa Luca, A.P., Crompton, A.W., Charig, A.J., 1976. A complete skeleton of the late Triassic ornithischian Heterodontosaurus tucki. Nature 264, 324-328.

Schaltegger, U., Guex, J., Bartolini, A., Schoene, B., Ovtcharova, M., 2008. Precise U-Pb age constraints for end-Triassic mass extinction, its correlation to volcanism and Hettangian post-extinction recovery. Earth and Planetary Science Letters 267. 266-275.

Schluter, D., 2000. The Ecology of Adaptive Radiation. Oxford University Press, Oxford.

Schoch, R., Wild, R., 1999. Die wirbeltier-Fauna im Keuper von Süddeutschland. In: Hauschke, N., Wilde, V. (Eds.), Trias, eine ganz andere Welt: Mitteleuropa im frühen Erdmittelalter. Verlag Dr. Friedrich Pfeil, München, pp. 395-408.

Schoene, B., Crowley, J.L., Condon, D.J., Schmitz, M.D., Bowring, S.A., 2006. Reassessing the uranium decay constants for geochronology using ID-TIMS U-Pb data. Geochimica et Cosmochimica Acta 70, 426-445.

Schultz, C.L., Scherer, C.M.d.S., Barberena, M.C., 2000. Bioestratigraphy of southern Brazilian Middle-Upper Triassic. Revista Brasileira de Geociencias 30, 495-498.

Schwartz, H.L., Gillette, D.D., 1994. Geology and taphonomy of the Coelophysis Quarry, Upper Triassic Chinle Formation, Ghost Ranch, New Mexico. Journal of Paleontology $68,1118-1130$

Seeley, H.G., 1888. On the classification of the fossil animals commonly named Dinosauria. Proceedings of the Royal Society of London 43, 165-171.

Sellwood, B.W., Valdes, P.J., 2006. Mesozoic climates; general circulation models and the rock record. Sedimentary Geology 190 (1-4), 269-287.

Sen, K., 2005. A new rauisuchian archosaur from the Middle Triassic of India. Palaeontology 48, 185-196.

Sepkoski Jr., J.J., 1996. Competition in macroevolution: the double wedge revisited. In: Jablonski, D., Erwin, D.H., Lipps, J.H. (Eds.), Evolutionary Paleobiology. University of Chicago Press, Chicago, IL, pp. 211-255.

Sereno, P.C., 1986. Phylogeny of the bird-hipped dinosaurs (Order Ornithischia). National Geographic Research 2, 234-256.

Sereno, P.C., 1991a. Basal archosaurs: phylogenetic relationships and functional implications. Society of Vertebrate Paleontology Memoir 2, 1-53.

Sereno, P.C., 1991b. Lesothosaurus, "fabrosaurids," and the early evolution of Ornithischia. Journal of Vertebrate Paleontology 11, 168-197.

Sereno, P.C., 1993. The pectoral girdle and forelimb of the basal theropod Herrerasaurus ischigualastensis. Journal of Vertebrate Paleontology 13, 425-450.

Sereno, P.C., 1997. The origin and evolution of dinosaurs. Annual Review of Earth and Planetary Sciences 25, 435-489.

Sereno, P.C. 1998. A rationale for phylogenetic definitions, with application to the higher-level taxonomy of Dinosauria. Neues Jahrbuch für Geologie und Paläontologie Abhandlungen 210, 41-83.

Sereno, P.C., 1999. The evolution of dinosaurs. Science 284, 2137-2147.

Sereno, P.C., 2005. The logical basis of phylogenetic taxonomy. Systems Biology 54, 595-619. 
Sereno, P.C., 2007a. The phylogenetic relationships of early dinosaurs: a comparative report. Historical Biology 19, 145-155.

Sereno, P.C., 2007b. Basal Sauropodomorpha: historical and recent phylogenetic hypotheses, with comments on Ammosaurus major (Marsh, 1889). Special Papers in Palaeontology 77, 261-289.

Sereno, P.C., Arcucci, A.B., 1994a. Dinosaurian precursors from the Middle Triassic of Argentina: Lagerpeton chanarensis. Journal of Vertebrate Paleontology 13, 385-399.

Sereno, P.C., Arcucci, A.B., 1994b. Dinosaurian precursors from the Middle Triassic of Argentina: Marasuchus lilloensis, gen. nov. Journal of Vertebrate Paleontology 14, $53-73$

Sereno, P.C., Novas, F.E., 1992. The complete skull and skeleton of an early dinosaur. Science 258, 1137-1140.

Sereno, P.C., Novas, F.E., 1994. The skull and neck of the basal theropod Herrerasaurus ischigualastensis. Journal of Vertebrate Paleontology 13, 451-476.

Sereno, P.C., Wild, R., 1992. Procompsognathus: theropod, "thecodont" or both? Journal of Vertebrate Paleontology 12, 435-458.

Sereno, P.C., Forster, C.A., Rogers, R.R., Monetta, A.M., 1993. Primitive dinosaur skeleton from Argentina and the early evolution of Dinosauria. Nature 361, 64-66.

Sereno, P.C., McAllister, S., Brusatte, S.L., 2005. TaxonSearch: a relational database for suprageneric taxa and phylogenetic definitions. PhyloInformatics 8, 1-21.

Sertich, J.J.W., Loewen, M.A., 2010. A new basal sauropodomorph dinosaur from the Lower Jurassic Navajo Sandstone of southern Utah. PLoS One 5, e9789, 1-17.

Sharov, A.G., 1970. An unusual reptile from the Lower Triassic of Fergana. Paleontological Journal 1970, 112-116.

Shipman, T.C., 2004. Links between sediment accumulation rates and the development of alluvial architecture: Triassic Ischigualasto Formation, northwestern Argentina. PhD Thesis, University of Arizona, Tuscon, 187 pp.

Shubin, N.H., Olsen, P.E., Sues, H.-D., 1994. Early Jurassic small tetrapods from the McCoy Brook Formation of Nova Scotia, Canada. In: Fraser, N.C., Sues, H.-D. (Eds.), In the Shadow of the Dinosaurs: Early Mesozoic Tetrapods. Cambridge University Press, Cambridge, pp. 244-250.

Sidor, C., Angielczyk, K., Hopson, J., Nesbitt, S., Smith, R., 2008. New vertebrate fossils from the Permo-Triassic Ruhuhu Basin of Tanzania. Journal of Vertebrate Paleontology 28 (3 Supplement), 142A.

Simmons, D.J., 1965. The non-therapsid reptiles of the Lufeng Basin, Yunnan, China. Fieldiana: Geology 15, 1-93.

Simms, M.J., Ruffell, A.H., 1990a. Climatic and biotic change in the Late Triassic. Journal of the Geological Society of London 147, 321-327.

Simms, M.J., Ruffell, A.H., 1990b. Synchroneity of climatic change and extinctions in the late Triassic. Geology 17, 265-268.

Smith, R., Kitching, J., 1997. Sedimentology and vertebrate taphonomy of the Tritylodon Acme Zone: a reworked palaeosol in the Lower Jurassic Elliot Formation, Karoo Supergroup, South Africa. Palaeogeography, Palaeoclimatology, Palaeoecology 131, 29-50.

Smith, N.D., Pol, D., 2007. Anatomy of a basal sauropodomorph dinosaur from the Early Jurassic Hanson Formation of Antarctica. Acta Palaeontologica Polonica 52, 657-674.

Smith, N.D., Makovicky, P.J., Hammer, W.R., Currie, P.J., 2007. Osteology of Cryolophosaurus ellioti (Dinosauria: Theropoda) from the Early Jurassic of Antarctica and implications for early theropod evolution. Zoological Journal of the Linnean Society $151,377-421$.

Stanley, G.D., 2006. Photosymbiosis and the evolution of modern coral reefs. Science $312,857-858$.

Stanley, S.M., Yang, X., 1994. A double mass extinction at the end of the Paleozoic Era. Science 266, 1340-1344

Stauffer, R.C. (Ed.), 1975. Charles Darwin's Natural Selection; Being the Second Part of His Big Species Book Written from 1836 to 1858. Cambridge University Press, Cambridge.

Stayton, C.T., 2006. Testing hypotheses of convergence with multivariate data: morphological and functional convergence among herbivorous lizards. Evolution 60, 824-841.

Sues, H.-D., Clark, J.M., Jenkins Jr., F.A., 1994. A review of the Early Jurassic tetrapods from the Glen Canyon Group of the American Southwest. In: Fraser, N.C., Sues, H.-D. (Eds.), In the Shadow of the Dinosaurs: Early Mesozoic Tetrapods. Cambridge University Press, Cambridge, pp. 284-294.

Sullivan, R.M., Lucas, S.G., 1999. Eucoelophysis baldwini, a new theropod dinosaur from the Upper Triassic of New Mexico, and the status of the original types of Coelophysis. Journal of Vertebrate Paleontology 19, 81-90.

Tabor, N.J., Montañez, I.P., Zierenberg, R., Currie, B.S., 2004. Mineralogical and geochemical evolution of a basalt-hosted fossil soil (Late Triassic, Ischigualasto Formation, northwest Argentina): potential for paleoenvironmental reconstruction. Geological Society of America Bulletin 116, 1280-1293.

Tabor, N.J., et al., 2006. A Late Triassic soil catena: landscape and climate controls on paleosol morphology and chemistry across the Carnian-age Ischigualasto-Villa Union Basin, northwestern Argentina. Geological Society of America Special Paper 416, 17-41.

Talbot, M., 1911. Podokesaurus holyokensis, a new dinosaur from the Triassic of the Connecticut valley. American Journal of Science 31, 469-479.

Taquet, P., 1984. Two new Jurassic specimens of coelurosaurs (Dinosauria). In: Hecht, M.K., Ostrom, J.H., Viohl, G., Wellnhofer, P. (Eds.), The Beginning of Birds. Freudnde des Jura-Museums, Eichstätt, pp. 229-232.

Tatarinov, L.P., 1984. New data on a collection of tetrapod teeth from the Rhaetian of Hallau (Switzerland). Paleontological Journal 1, 138-140.

Thulborn, R.A., 1970a. The skull of Fabrosaurus australis, a Triassic ornithischian dinosaur. Palaeontology 13, 414-432.

Thulborn, R.A., 1970b. The systematic position of the Triassic ornithischian dinosaur Lycorhinus angustidens. Zoological Journal of the Linnean Society 49, 235-245.
Thulborn, R.A., 1971. Tooth wear and jaw action in the Triassic ornithischian dinosaur Fabrosaurus. Journal of Zoology 164, 165-179.

Thulborn, R.A., 1972. The postcranial skeleton of the Triassic ornithischian dinosaur Fabrosaurus australis. Palaeontology 15, 29-60.

Thulborn, R.A., 1974. A new heterodontosaurid dinosaur (Reptilia: Ornithischia) from the Upper Triassic Red Beds of Lesotho. Zoological Journal of the Linnean Society 55, 151-175.

Thulborn, R.A., 1975. Dinosaur polyphyly and the classification of archosaurs and birds. Australian Journal of Zoology 23 (2), 249-270.

Thulborn, T., 2006. On the tracks of the earliest dinosaurs: implications for the hypothesis of dinosaurian monophyly. Alcheringa 30, 273-311.

Tucker, M.E., Benton, M.J., 1982. Triassic environments, climates and reptile evolution. Palaeogeography, Palaeoclimatology, Palaeoecology 40, 361-379.

Tykoski, R.S., 1997. The osteology of Syntarsus kayentakatae and its implications for ceratosaurid phylogeny. MSc Dissertation, University of Texas, Austin, TX

Tykoski, R.S., 2005. Vertebrate paleontology in the Arizona Jurassic. Mesa Southwest Museum Bulletin 11, 72-93.

Tykoski, R.S., Rowe, T., 2004. Ceratosauria, In: Weishampel, D.B., Dodson, P., Osmólska, H. (Eds.), The Dinosauria, 2nd edition. University of California Press, Berkeley, CA, pp. 47-70.

Tykoski, R.S., Rowe, T.B., Ketcham, R.A., Colbert, M.W., 2002. Calsoyasuchus valliceps, a new crocodyliform from the Early Jurassic Kayenta Formation of Arizona. Journal of Vertebrate Paleontology 22, 593-611.

Upchurch, P., Hunn, C.A., Norman, D.B., 2002. An analysis of dinosaurian biogeography: evidence for the existence of vicariance and dispersal patterns caused by geological events. Proceedings of the Royal Society of London, Series B 269, 613-621.

Upchurch, P., Barrett, P.M., Galton, P.M., 2007a. A phylogenetic analysis of basal sauropodomorph relationships: implications for the origin of sauropod dinosaurs. Special Papers in Palaeontology 77, 57-90.

Upchurch, P., Barrett, P.M., Zhao, X., Xu, X., 2007b. A re-evaluation of Chinshakiangosaurus chunghoensis Ye vide Dong 1992 (Dinosauria, Sauropodomorpha): implications for cranial evolution in basal sauropod dinosaurs. Geological Magazine 144 247-262.

Valentine, J.W., 1980. Determinants of diversity in higher taxonomic categories. Paleobiology 6, 444-450.

Van Valen, L.M., 1973. A new evolutionary law. Evolutionary Theory 1, 1-30.

von Huene, F.R., 1908. Die dinosaurier der europäischen Triasformationen mit berucksichtigung der aussereuropäischen vorkommnisse. Geologische und Paläontologische Abhandlungen, Supplement 1, 1-419.

von Huene, F.R., 1932. Die fossile Reptil-Ordnung Saurischia, ihre Entwicklung und Geschichte. Monographien zur Geologie und Paläontologie 4, 1-361.

von Huene, F., 1934. Ein neurer Coelurosaurier in der thüringischen Trias. Paläontologishe Zeitscrift 16, 145-170.

von Meyer, H., 1837. Mitteilung an Prof. Bronn (Plateosaurus engelhardti). Neues Jahrbuch für Mineralogie, Geologie, und Paläontologie 1837, 817.

Wagner, P.J., 1997. Patterns of morphologic diversification among the Rostroconchia. Paleobiology 23, 115-150.

Waldman, M., 1974. Megalosaurids from the Bajocian (Middle Jurassic) of Dorset. Palaeontology 17, 325-339.

Walker, J.D., Geissman, J.W., 2009. 2009 GSA Geologic Time Scale. GSA Today 19 (4), 60-61.

Wang, S.C., Dodson, P., 2006. Estimating the diversity of dinosaurs. Proceedings of the National Academy of Sciences of the United States of America 103, 13601-13605.

Wang, X., Zhou, Z., Zhang, F., Xu, W., 2002. A nearly complete articulated rhamphorhynchoid pterosaur with exceptionally well-preserved wing membranes and "hairs" from Inner Mongolia, Northeast China. Chinese Science Bulletin 47, 226-232.

Weinbaum, J.C., Hungerbühler, A., 2007. A revision of Poposaurus gracilis (Archosauria: Suchia) based on two new specimens from the Late Triassic of the southwestern U.S.A Paläontologische Zeitschrift 81, 131-145.

Weishampel, D.B., Witmer, L.M., 1990. Heterodontosauridae. In: Weishampel, D.B. Dodson, P., Osmólska, H. (Eds.), The Dinosauria. University of California Press, Berkeley, CA, pp. 486-497.

Weishampel, D.B., Barrett, P.M., Coria, R.A., Le Loeuff, J., Xu, X., Zhao, X., Sahni, A Gomani, E.M.P., Noto, C.R., 2004. Dinosaur distribution, In: Weishampel, D.B. Dodson, P., Osmólska, H. (Eds.), The Dinosauria, 2nd edition. University of California Press, Berkeley, CA, pp. 517-606.

Welles, S.P., 1954. New Jurassic dinosaur from the Kayenta Formation of Arizona. Bulletin of the Geological Society of America 65, 591-598.

Welles, S.P., 1970. Dilophosaurus (Reptilia: Saurischia), a new name for a dinosaur. Journal of Paleontology 44, 989.

Welles, S.P., 1984. Dilophosaurus wetherilli (Dinosauria, Theropoda): osteology and comparisons. Palaeontographica Abteilung A 185, 85-180.

Welles, S.P., 1986. Thoughts on the origin of the Theropoda. In: Padian, K. (Ed.), The Beginning of the Age of Dinosaurs: Faunal Change across the Triassic-Jurassic Boundary. Cambridge University Press, Cambridge, pp. 31-34.

Whiteside, D.I., Marshall, J.E.A., 2008. The age, fauna and palaeoenvironment of the Late Triassic fissure deposits of Tytherington, south Gloucestershire, UK. Geological Magazine 145, 105-147.

Whiteside, J.H., Olsen, P.E., Eglinton, T., Brookfield, M.E., Sambrotto, R.N., 2010 Compound-specific carbon isotopes from Earth's largest flood basalt province directly link eruptions to the end-Triassic mass extinction. Proceedings of the National Academy of Science 107 (15), 6721-6725.

Wills, M.A., Briggs, D.E.G., Fortey, R.A., 1994. Disparity as an evolutionary index: a comparison of Cambrian and Recent arthropods. Paleobiology 20, 93-131.

Wills, M.A., Barrett, P.M., Heathcote, J.F., 2008. The modified Gap Excess Ratio (GER*) and the stratigraphic congruence of dinosaur phylogenies. Systems Biology 57, 891-904. 
Xu, X., Zhang, F., 2005. A new maniraptoran dinosaur from China with long feathers on the metatarsus. Die Naturwissenschaften 92, 173-177.

Xu, X., Tang, Z.-L., Wang, X.-L., 1999a. A therizinosauroid dinosaur with integumentary structures from China. Nature 399, 350-354.

Xu, X., Wang, X.-L., Wu, X.-C., 1999b. A dromaeosaurid dinosaur with a filamentous integument from the Yixian Formation of China. Nature 401, 262-266.

Xu, X., Zhao, X.-J., Clark, J.M., 2001. A new therizinosaur from the Lower Jurassic lower Lufeng Formation of Yunnan, China. Journal of Vertebrate Paleontology 21, 477-483.

Xu, X., Norell, M.A., Kuang, X., Wang, X., Zhao, Q., Jia, C., 2004. Basal tyrannosauroids from China and evidence for protofeathers in tyrannosauroids. Nature 431, 680-684

Xu, X., Forster, C.A., Clark, J.M., Mo, J., 2006. A basal ceratopsian with transitional features from the Late Jurassic of northwestern China. Proceedings of the Royal Society of London, Series B 273, 2135-2140.

$\mathrm{Xu}$, X., et al., 2009. A Jurassic ceratosaur from China helps clarify avian digital homologies. Nature 459, 940-944.

Yadagiri, P., 1988. A new sauropod Kotasaurus yamanpalliensis from Lower Jurassic Kota Formation of India. Journal of the Geological Society of India 11, 102-127.

Yadagiri, P., 2001. The osteology of Kotasaurus yamanpalliensis, a sauropod dinosaur from the Early Jurassic Kota Formation of India. Journal of Vertebrate Paleontology $21,242-252$.

Yates, A.M., 2003a. A new species of the primitive dinosaur Thecodontosaurus (Saurischia: Sauropodomorpha) and its implications for the systematics of early dinosaurs. Journal of Systematic Palaeontology 1, 1-42.

Yates, A.M., 2003b. The species taxonomy of the sauropodomorph dinosaurs from the Löwenstein Formation (Norian, Late Triassic) of Germany. Palaeontology 46, 317-337.

Yates, A.M., 2004. Anchisaurus polyzelus (Hitchcock): the smallest known sauropod dinosaur and the evolution of gigantism among sauropodomorph dinosaurs. Postilla $230,1-58$.

Yates, A.M., 2005. A new theropod dinosaur from the Early Jurassic of South Africa and its implications for the early evolution of theropods. Palaeontologia Africana 41, 105-122.
Yates, A.M., 2007a. The first complete skull of the Triassic dinosaur Melanorosaurus Haughton (Sauropodomorpha: Anchisauria). Special Papers in Palaeontology 77 $9-55$

Yates, A.M., 2007b. Solving a dinosaurian puzzle: the identity of Aliwalia rex Galton. Historical Biology 19, 93-123.

Yates, A.M., Kitching, J.W., 2003. The earliest known sauropod dinosaur and the first steps towards sauropod locomotion. Proceedings of the Royal Society of London, Series B 270, 1753-1758.

Yates, A.M., Bonnan, M.F., Neveling, J., Chinsamy, A., Blackbeard, M.G., 2010. A new transitional sauropodomorph dinosaur from the Early Jurassic of South Africa and the evolution of sauropod feeding and quadrupedalism. Proceedings of the Royal Society of London, Series B 277, 787-794.

Young, C.-C., 1941. A complete osteology of Lufengosaurus huenei Young (gen. et sp. nov.) from Lufeng, Yunnan, China. Palaeontologia Sinica, New Series C 131, 1-53.

Young, C.-C., 1942. Yunnanosaurus huangi Young (gen. et sp. nov.), a new Prosauropoda from the red beds at Lufeng, Yunnan. Bulletin of the Geological Society of China 22 63-104.

Zerfass, H., Chemale Jr., F., Schultz, C.L., Lavina, E., 2004. Tectonics and sedimentation in southern South America during Triassic. Sedimentary Geology 166, 265-292.

Zhang, Y., Yang, Z., 1994. A New Complete Osteology of Prosauropoda in Lufeng Basin Yunnon [sic] China: Jingshanosaurus. Yunnan Publishing House of Science and Technology, Kunming, China. 98 pp.

Zhang, F., Zhou, Z., Xu, X., Wang, X.-L., 2002. A juvenile coelurosaurian theropod from China indicates arboreal habits. Die Naturwissenschaften 89, 394-398.

Zhang, F., Zhou, Z., Xu, X., Wang, X.-L., Sullivan, C., 2008. A bizarre Jurassic maniraptoran from China with elongate ribbon-like feathers. Nature 455, 1105-1108.

Zhang, F., Kearns, S.L., Orr, P.J., Benton, M.J., Zhou, Z., Johnson, D., Xu, X., Wang, X., 2010 Fossilized melanosomes and the colour of Cretaceous dinosaurs and birds. Nature 463, 1075-1078.

Zhao, X.-J., Xu, X., 1998. The oldest coelurosaurian. Nature 394, 234-235.

Zheng, X.-T., You, H.-L., Xu, X., Dong, Z.-M., 2009. An Early Cretaceous heterodontosaurid dinosaur with filamentous integumentary structures. Nature 458 333-336. 